\title{
Ratiometric fluorescence imaging and marker-free motion tracking of Langendorff perfused beating rabbit hearts
}

\author{
Dissertation \\ for the award of the degree \\ "Doctor of Philosophy Ph.D." \\ Division of Mathematics and Natural Sciences \\ of the Georg-August-Universität Göttingen
}

within the doctoral program in Physics

of the Georg-August University School of Science (GAUSS)

\author{
submitted by \\ Vineesh Kappadan \\ from Mattannur, India
}

Göttingen, 2020 


\section{Thesis Committee}

APl. Prof. Dr. Ulrich Parlitz,

Forschungsgruppe Biomedizinische Physik,

Max-Planck-Institut für Dynamik und Selbstorganisation

PROF. DR. JÖRG ENDERLEIN,

Drittes Physikalisches Institut - Biophysik,

Georg-August-Universität Göttingen

Prof. Dr. MARTin UECKeR,

Institut für Diagnostische und Interventionelle Radiologie, Universitätsmedizin Göttingen

\section{Members of the Examination Board}

Referee: APl. Prof. Dr. Ulrich Parlitz,

Forschungsgruppe Biomedizinische Physik,

Max-Planck-Institut für Dynamik und Selbstorganisation

$2^{\text {nd }}$ Referee: Prof. DR. JÖRG ENDERLEIN,

Drittes Physikalisches Institut - Biophysik,

Georg-August-Universität Göttingen

Further members of the Examination Board:

Prof. Dr. STEFAn Luther,

Forschungsgruppe Biomedizinische Physik,

Max-Planck-Institut für Dynamik und Selbstorganisation

Prof. Dr. MARTIN UECKeR,

Institut für Diagnostische und Interventionelle Radiologie, Universitätsmedizin Göttingen 
Prof. Dr. Florentin WÖRGÖTter,

Drittes Physikalisches Institut - Biophysik, Georg-August-Universität Göttingen

APl. Prof. Dr. Alexander Egner, Laser-Laboratorium Göttingen e.V. (LLG), Georg-August-Universität Göttingen

Date of oral examination: 2020-07-14 



\section{Abstract}

Optical mapping is a fluorescence based imaging technique used extensively in cardiac research to study the electrophysiological properties of isolated hearts kept at physiological conditions. The major limitation of optical mapping studies are the distortions of electrophysiological signals due to the contractile motion of the hearts. To reduce electrophysiological signal distortions due to the contractile motion artifacts, the mechanical motion has been suppressed in optical mapping experiments using electromechanical uncouplers such as Blebbistatin. Recent studies used marker-free numerical motion tracking and motion stabilization techniques to record electrophysiological parameters in the absence of electromechanical uncouplers and showed that contractile motion of the cardiac tissue is no longer a limitation in optical mapping studies. However, despite these developments, accurate measurements of quantities such as action potential duration (APD) and cardiac restitution are still challenging due to the residual motion artifacts present in the electrophysiological signals even after numerical motion tracking.

A combination of marker-free motion tracking and ratiometric optical mapping technique is used in this thesis to minimize motion-related artifacts from contracting hearts. This combined experimental and numerical technique reduced motion artifacts significantly and hence, the combination is used to precisely measure APD, cardiac restitution and ventricular fibrillation frequencies from Langendorff perfused contracting and deforming rabbit hearts. A systematic comparison of these electrophysiological parameters in contracting and Blebbistatin-uncoupled conditions showed, on average, $27 \pm 5 \%$ ( $N=5$ hearts) shortening of APD in contracting hearts as compared to Blebbistatin-uncoupled hearts. Ventricular fibrillation frequency significantly increased in contracting hearts $(13 \pm 3.5 \mathrm{~Hz})$ in comparison with Blebbistatin-uncoupled hearts $(8 \pm 1.5 \mathrm{~Hz})$. 



\section{Contents}

1 Introduction 1

2 Background of cardiac electrophysiology 5

2.1 Electrical conduction system of the heart . . . . . . . . 5

2.2 Cardiac arrhythmia . . . . . . . . . . . . . 5

2.3 The Langendorff perfused hearts . . . . . . . . . 7

2.4 Study of electromechanical interactions . . . . . . . . . . 9

2.4.1 Excitation-contraction coupling ......... . 9

2.4.2 Mechano electric feedback . . . . . . . . . . . 11

2.5 Optical mapping of cardiac electrophysiology . . . . . . . . 11

2.5.1 Motion artifacts . . . . . . . . . . . . 13

2.5.2 Excitation-contraction uncoupling . . . . . . . 15

3 Experimental methods 17

3.1 Ratiometry .................... 17

3.1 .1 Excitation ratiometry . . . . . . . . . . 19

3.2 Experimental setup and description . . . . . . . . . . . 21

3.3 Optical mapping data processing . . . . . . . . . . . . 23

3.3.1 Spatio-temporal smoothing . . . . . . . . 24

3.3.2 Sliding window normalization . . . . . . . . 26

4 Motion artifacts reduction techniques 29

4.1 Marker-free motion tracking . . . . . . . . . . . . . . . 29

4.1.1 Motion compensation . . . . . . . . . . . 35

4.2 Action potential wave propagation in motion tracked hearts . 36

4.3 Marker-free motion tracking and excitation ratiometry . . . . 38

4.4 Comparison of action potential time traces between contracting and Blebbistatin-

uncoupled hearts . . . . . . . . . . . . . 42 


\section{Comparison of APD restitution in}

contracting and Blebbistatin-uncoupled hearts 45

5.1 APD and electrical restitution . . . . . . . . . . . . . 45

5.1.1 Dynamic restitution in optical mapping . . . . . . . . 47

5.2 Comparison of APDs in contracting and Blebbistatin-uncoupled hearts during electrical pacing . . . . . . . . . . . . . . . 49

5.3 Comparison of restitution curves between contracting and Blebbistatin-uncoupled hearts . . . . . . . . . . . . 51

5.4 Variation of action potential duration and motion amplitude during electrical pacing . . . . . . . . . . . . 53

5.5 Comparison of APD maps in contracting and Blebbistatinuncoupled hearts . . . . . . . . . . . . . . . 54

5.6 Electromechanical restitution curves from multiple hearts . . . 56

5.7 Variation of action potential duration and restitution properties with Blebbistatin concentration . . . . . . . . . . . 58

5.8 Variation of action potential duration with Blebbistatin washout 61

5.9 Impact of pacing frequency on electromechanical coupling . . 62

6 Comparison of ventricular fibrillation dynamics in contracting and Blebbistatin-uncoupled hearts $\quad 65$

6.1 Induction of ventricular fibrillation $\ldots \ldots \ldots 6$

6.2 Dominant and weighted dominant frequencies of ventricular fibrillation . . . . . . . . . . . . . 66

6.3 Variation of ventricular fibrillation frequencies with Blebbistatin concentration . . . . . . . . . . . . . 73

6.4 Ventricular fibrillation frequencies from multiple hearts . . . 75

6.5 Variation of ventricular fibrillation frequencies with Blebbistatin washout . . . . . . . . . . . . . . . 77

6.6 Comparison of ventricular fibrillation frequencies calculated from ECG and optical data . . . . . . . . . . . . . 78

$\begin{array}{lll}7 & \text { Summary and discussion } & \mathbf{8 1}\end{array}$

7.1 Limitations . . . . . . . . . . . . . 83

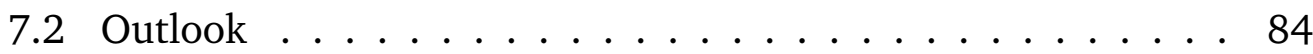

$\begin{array}{ll}\text { Bibliography } & 87\end{array}$ 


\section{Introduction}

The heart is a vital organ in the human body responsible for pumping blood in an efficient manner. An adult heart pumps about 6000-7500 liters of blood daily hence making the life possible. At the cellular level, the mechanical contraction of the cardiomyocyte (heart muscle cell) is triggered by the cardiac action potential, which is a brief change in membrane potential across the cell membrane of cardiac cells. A rapid positive change in membrane potential (depolarization) is followed by a plateau phase and it finally followed by a slow decrease in membrane potential (repolarization). The action potential triggers the release of calcium stored in the Sarcoplasmic Reticulum (SR) and the calcium released from the SR triggers the mechanical contraction of the cardiomyocyte. This entire process in which electrical excitation produces mechanical contraction through the release of calcium is called excitationcontraction coupling (ECC). Multiple studies [Sir96; Fra96; Fra00] show that electromechanical interactions are bidirectionally coupled such that in addition to excitation-contraction coupling, mechanical perturbations of the heart can produce electrical excitations through a process called mechano-electric feedback (MEF). Studies of these electromechanical interactions are essential in understanding lethal cardiac arrhythmias such as ventricular fibrillation (VF).

A widely used non contact technique to study electrical excitation of the cardiac tissue is called optical mapping (basic overviews can be found in [Min11; ENS04]). Optical mapping is a fluorescence imaging technique used extensively in cardiac research to image and visualize electrophysiological properties such as action potential [SC00], intracellular calcium concentration [Nid+98; Ven+12] or both [LS01; Lee+12] from the surface of isolated hearts kept at physiological conditions. Optical mapping is used to obtain high spatial and temporal resolution of action potential or calcium concentration signals from the surface of the heart by loading it with fast responding voltage or calcium sensitive fluorescent dyes. Upon excitation with proper wavelength of light, the fluorescent emission from these dyes 
undergo intensity modulation due to the electrophysiological quantities under study. For example, voltage sensitive fluorescent dyes undergo a spectral shift upon depolarization of the cardiac tissue with action potential upstroke. These fluorescent emissions containing the information of action potential or calcium concentration are captured by a high resolution camera. Though optical mapping is considered as the gold standard technique during the past 25 years, like all other techniques, optical mapping also has limitations. Optical mapping studies are highly susceptible to motion and even a small motion of the heart tissue can distort the fluorescence signal captured on the detector. This so called motion artifacts are the biggest challenge in optical mapping studies involving beating hearts.

To overcome the difficulties of motion artifacts, until recent times, all optical mapping studies were carried out by suppressing the mechanical contraction of the heart by using either mechanical constraints or with the help of pharmacological electromechanical uncouplers such as DAM [Liu+93], Cytocholasin-D [Ket+04b], Blebbistatin [Fed+07] etc. These uncouplers uncouple the electrical and mechanical activity by suppressing the contractile motion. This enables the measurement of action potential and calcium concentration from the cardiac surface which are free from motion artifacts. Among these pharmacological uncouplers, Blebbistatin is the most commonly used uncoupler in optical mapping studies for the last 10 years and it is considered as the most reliable pharmacological agent with minimal effects on cardiac electrophysiology [Fed+07]. However, studies involving Langendorff perfused hearts (detailed description in Chapter 2) show variations in electrophysiological properties such as action potential duration with Blebbistatin [Bra+13; Lee+19]. Apart from that, suppressing the mechanical contraction is not physiological and it does not allow the study of electromechanical interactions simultaneously. Recently, numerical motion tracking and motion compensation algorithms were developed to track and compensate the contractile motion of the cardiac tissue with polyethylene markers [Bou+11; Zha +16$]$ and also without using markers to track and stabilize the contractile motion in optical mapping studies [CL18; CSSL17]. Marker-free motion tracking was also combined with ultrasound imaging to study and characterize the chaotic wave pattern of ventricular fibrillation from contracting rabbit hearts $[\mathrm{Chr}+18]$. 
However, precise measurement of electrophysiological parameters such as action potential duration (APD) and electrical restitution (variation of APD with heart rate, also called APD restitution) from a contracting heart in optical mapping studies are still challenging due to residual motion artifacts present in the action potential signals even after tracking and stabilizing the contractile motion. Accurate measurements of these properties are highly important since APD alternans (beat to beat variations in APD) [Kar93; Kar94] and APD restitution [Xie+04; Ng16; Mon14] have dynamical consequences in the arrhythmogenicity of the heart. Also, drugs which reduce restitution slope such as bretylium, verapamil, and diacetyl monoxime (DAM) are shown to have antiarrhythmic behavior [Wei+02; RKG99]. Hence to measure the important electrophysiological properties of contracting hearts precisely, the residual artifacts need to be reduced. The main reasons for these residual artifacts are the spatial variation of local light intensity and spatio-temporal variation of local dye concentration within the heart, which cannot be removed by motion tracking alone. Ratiometric optical mapping addresses these limitations by canceling the common artifacts present in the electrophysiological signal $[\mathrm{Bac}+11]$. Hence, this thesis focuses on the combination of marker-free motion tracking and ratiometric optical mapping technique with the following goals:

1. Combining ratiometric optical mapping and marker-free motion tracking to obtain optical action potential signals with reduced motion artifacts.

2. Precise measurement of APD and electrical restitution from contracting hearts.

3. Systematic comparison of APD and electrical restitution of the contracting hearts with that of non-contracting hearts (electromechanically uncoupled with Blebbistatin)

4. Comparison of arrhythmic dynamics, more specifically ventricular fibrillation in contracting and uncoupled hearts.

The entire thesis is divided into 7 chapters including the introductory chapter. In chapter 2 , background information about cardiac electrophysiology including Langendorff perfusion setup, electromechanical interactions, optical mapping technique to measure the electro-physiological quantities and the challenges of optical mapping techniques are shown. Chapter 3 shows the detailed description of ratiometric optical mapping experiment. 
Chapter 4 focuses on motion artifact reduction techniques such as numerical motion tracking, ratiometry and the combination of both. Chapter 5 uses combined motion tracking and ratiometric techniques with a beating heart to precisely measure action potential duration (APD) and also to compare APD restitution curves of contracting and contraction inhibited (by the administration of Blebbistatin) hearts. Chapter 6 compares the dynamics of ventricular fibrillation in contracting and contraction inhibited hearts by measuring the dominant frequency of optical action potential signals using the combination of motion tracking and ratiometry. Chapter 7 summarizes the obtained results, limitations of the techniques used and also discuss possible improvements for the future. 


\section{Background of cardiac electrophysiology}

\subsection{Electrical conduction system of the heart}

The mechanical contraction of the heart chambers are triggered by electrical impulses (action potentials) originating from the sinoatrial (SA) node situated at the right atrium of the heart. The SA node undergoes self excitation and produces electrical impulses in a regular interval, hence it is called the natural pacemaker of the heart. The electrical impulses from the SA node excite the right atria and travels through the interatrial pathway to excite the left atria and trigger atrial contraction. The electrical impulses then propagate to the ventricles through the atrioventricular (AV) node. The AV node slows down the propagation of electric current to ensure that atria contract fully before ventricular excitation. After entering the ventricle, the electrical impulses further propagate through left and right bundle branches and finally through Purkinje fibers to trigger the contraction of left and right ventricles.

\subsection{Cardiac arrhythmia}

Cardiac arrhythmias (also known as heart rhythm disorders) are the malfunctioning of the electrical activity of the heart. This abnormal electrical activity causes abnormal contraction of the heart in contrast to the normal rhythm (sinus rhythm). There are mainly two types of cardiac arrhythmia: bradyarrhythmia and tachyarrhythmia. Bradyarrhythmia is a condition of the heart in which the heart rate becomes very low as compared to the normal heart rate (60-100 beats per minutes in human, athletes are exceptions). Tachyarrhythmia is characterized by fast heart rates. For an adult human, heart rates less than 60 beats per minute are considered as bradyarrhythmias and heart rates higher than 100 beats per minute are called tachyarrhythmias. 


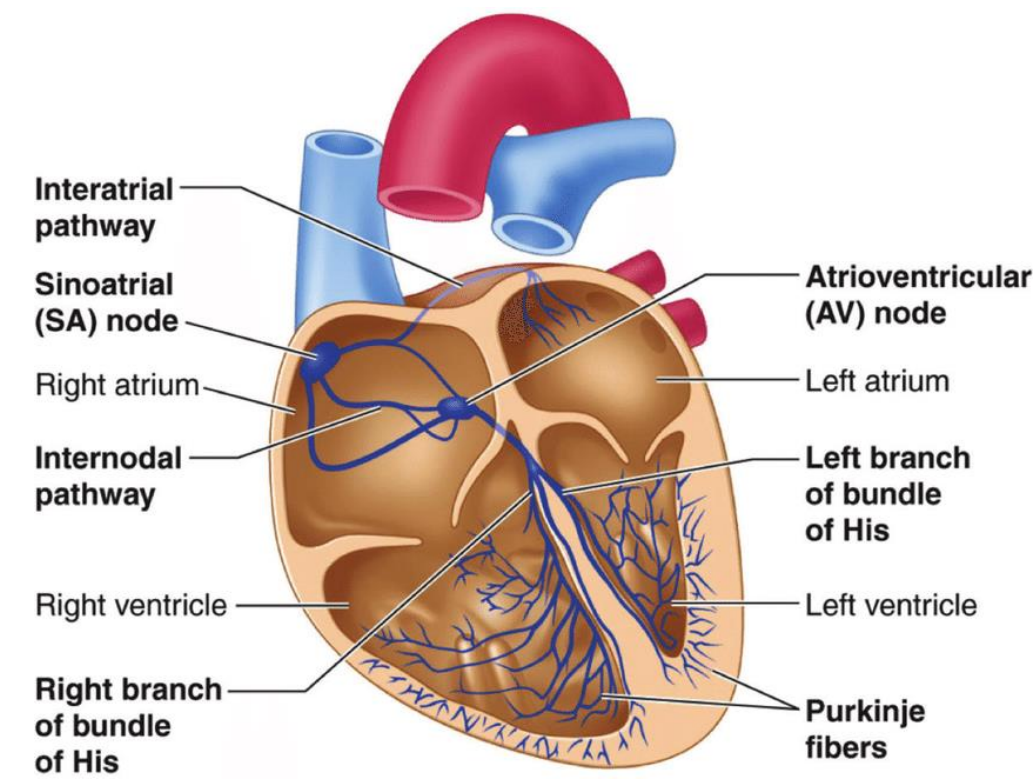

Fig. 2.1: Electrical conduction system of the heart. Electrical impulses originating from the SA node spread across the right and left atria and are responsible for atrial contraction. These impulses then propagate through the AV node to the ventricles, and propagate further through bundle branches and Purkinje fibers and finally trigger the ventricular contraction. Image reproduced from [Gan+07], Licence: Creative Commons Attribution 3.0.

Ventricular fibrillation (VF) is the most lethal tachyarrhythmia characterized by fast and irregular electrical activity in the lower chambers of the heart (ventricles). These electrical activities produce non coherent contraction in different parts of the cardiac tissue, hence the effective pumping power is lost. VF can cause sudden cardiac arrest within a short interval of time if untreated. Figure 2.2 shows exemplary time traces of optical action potentials obtained during normal and abnormal (here, ventricular fibrillation) heart rhythms. The sinus rhythm consists of regular and low frequency (here, frequency $<2 \mathrm{~Hz}$ ) action potential signals, whereas ventricular fibrillation is characterized by fast (here, frequency $\approx 9 \mathrm{~Hz}$ ) and irregular action potential time traces. In addition to the difference in temporal variations of the action potential signal, there are also notable differences in action potential wave propagation during normal and abnormal heart rhythms. The electrical activity propagates as a planar wave of excitation across the heart tissue during normal sinus rhythm, whereas electrical excitation of the heart during ventricular fibrillation consists of spatio-temporal chaos. 

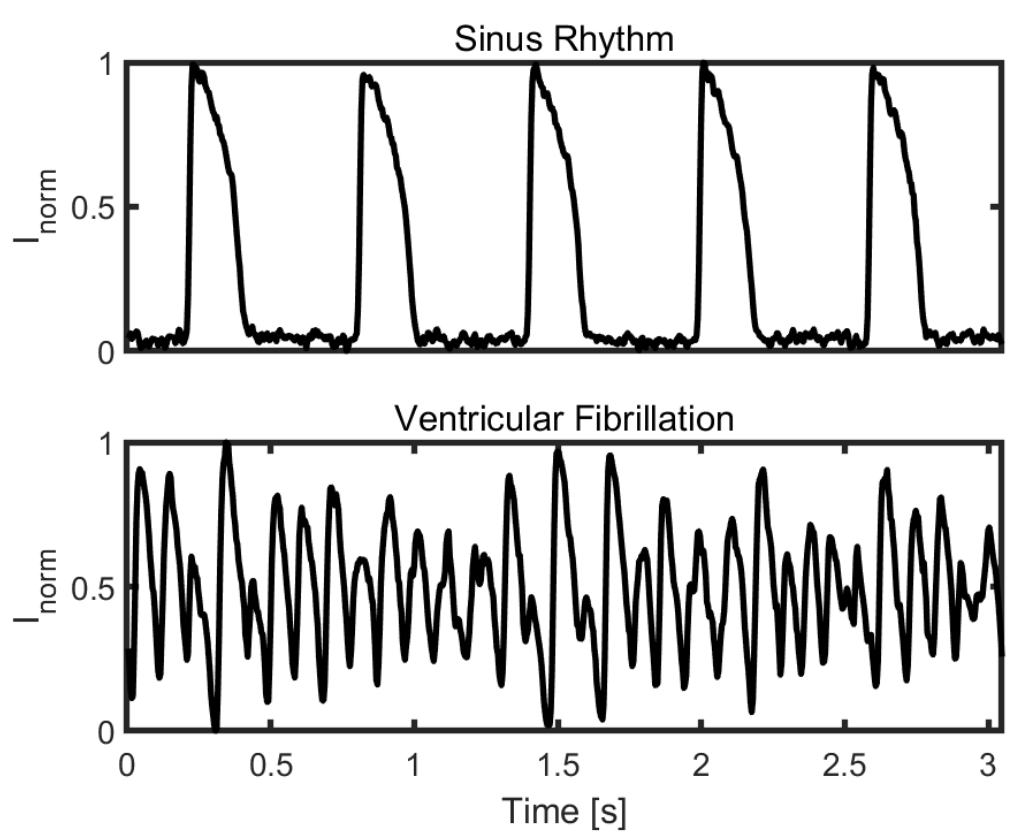

Fig. 2.2: Optical action potential time traces showing the variation of action potential modulated normalized fluorescent intensity $\left(I_{\text {norm }}\right)$ for normal (sinus rhythm) and abnormal (ventricular fibrillation) heart rhythms. The time traces were extracted from the left ventricular surface of a rabbit heart. The contraction of the heart was inhibited by Blebbistatin. The time traces were spatio-temporally filtered with a window size of $3 \times 3 \times 3$ (average of $3 \times 3$ pixels and 3 frames). Ventricular fibrillation is induced by high frequency electrical pulses with the help of a stimulating electrode placed on the surface of the heart.

\subsection{The Langendorff perfused hearts}

Langendorff perfused hearts, introduced in 1895 by Oscar Langendorff are isolated perfused mammalian hearts used widely in cardiac research to keep the hearts alive in a specific physiological conditions outside the body [BMY11]. Langendorff perfused hearts have been used to study myocardial physiology and contractility among a wide range of species including mice, rat, rabbit, dog, pig and human. All experimental studies in this thesis used Langendorff perfused rabbit hearts.

In a Langendorff perfusion setup as shown in Figure 2.3, the excised animal heart is perfused in a retrograde fashion via the aorta using Tyrode's solution. The solution provides the necessary nutrients and oxygen to the heart to keep it alive for many hours (usually 4-8 hours) in a physiological condition similar to blood. The Tyrode's solution is usually bubbled with 
carbogen containing 95\% oxygen and 5\% carbon dioxide to ensure enough oxygen and a physiological $\mathrm{PH}$ value $(\approx 7.4)$ to the heart. This solution is preheated and pumped into the bubble trap through a heat exchanger. The heart is connected directly below the bubble trap by the cannulation of aorta, which closes the aortic valve upon perfusion and hence, the perfusate passes through the coronary vasculature and nourishes the heart. The perfused heart is allowed to hang in a bath containing a heating facility, to maintain the required physiological temperature $\left(37^{\circ} \mathrm{C}\right)$. The Langendorff perfusion setup is comparatively simple and can be used to add drugs and fluorescent dyes to the heart without many complications. Also, the electrophysiological parameters such as electrocardiogram (ECG) and optical action potential etc. can be measured with ease in a Langendorff perfusion setup.

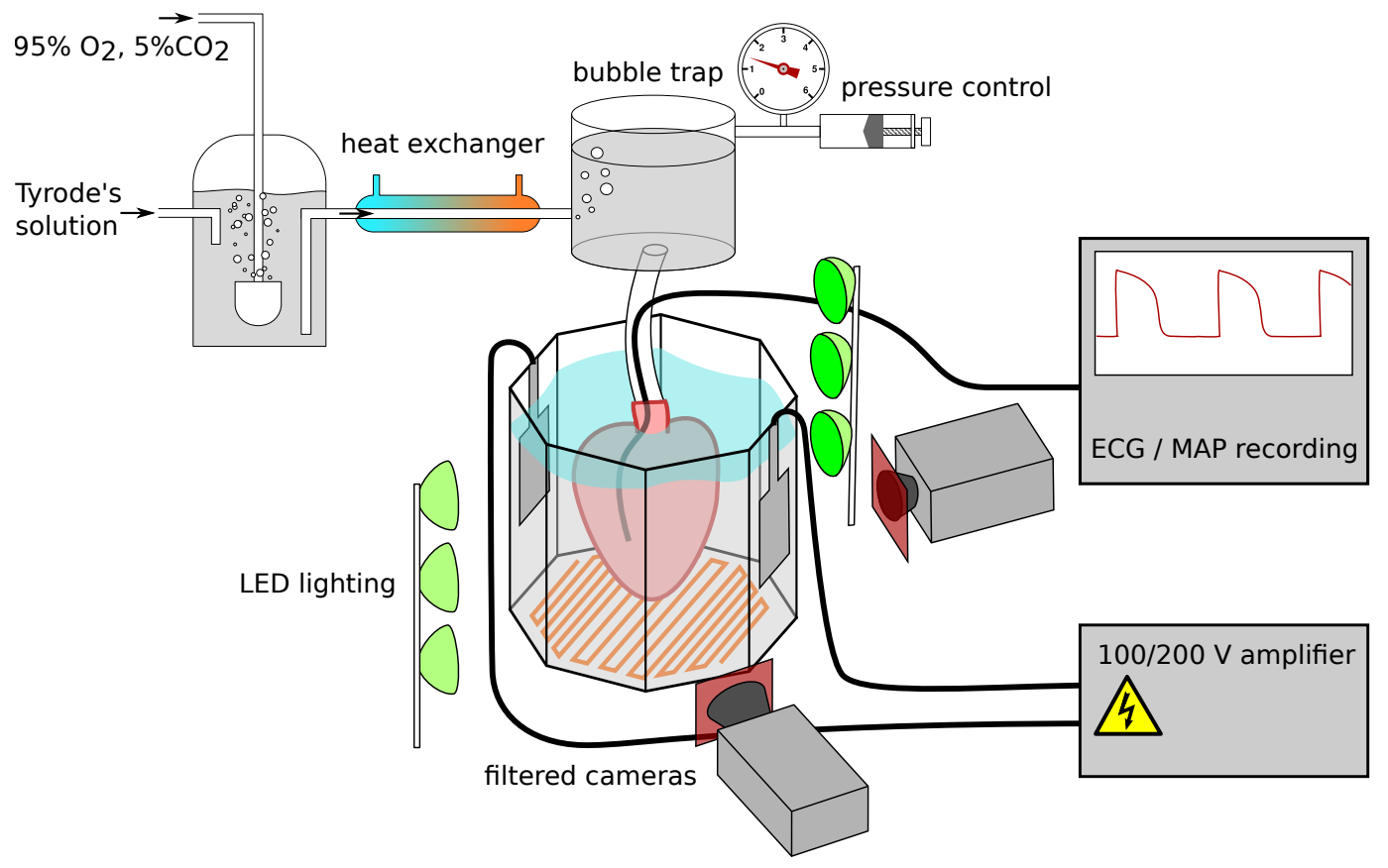

Fig. 2.3: Schematic of Langendorff perfused heart. The extracted heart from the animal is kept submerged in a perfusion bath containing Tyrode's solution. Voltage sensitive or calcium sensitive fluorescent dyes can be provided to the heart tissue through the bubble trap in a retrograde fashion. LEDs and camera are used for optical mapping and ECG signals are captured with a combination of ECG electrodes and an amplifier. Two pedal electrodes connected to a 100/200 $\mathrm{V}$ amplifier are used to provide electrical shocks to terminate arrhythmias (defibrillation). Sketch from [Hor13]. 


\subsection{Study of electromechanical interactions}

The interactions between electrical and mechanical activities of cardiac muscle cells are bidirectional phenomena (an overview can be found in [PT14]) in which electrical excitation of the cardiomyocyte triggers its mechanical contraction through excitation-contraction coupling (ECC) and mechanical perturbation produces electrical depolarization through a process called mechano-electric feedback (MEF). Hence, simultaneous measurement of electrical and mechanical activities of the heart can provide useful information regarding these complex electromechanical interactions. However these electromechanical properties often vary spatially (across the heart) and temporally. For example, ventricular fibrillation dynamics as mentioned before shows spatio-temporal chaotic activities. Despite active research, electromechanical interactions of a beating heart are still not fully understood mainly due to the technical difficulties to precisely measure both electrical and mechanical properties of the beating heart simultaneously from multiple locations of the heart tissue. Though optical mapping provides a smart way of measuring electrical activity from multiple locations of the cardiac tissue, often mechanical contraction is uncoupled from electrical excitation by using pharmacological agents to reduce artifacts originating from the contractile motion. However, recent studies [Bou+11; Zha+16; CSSL17; CL18] used numerical post processing algorithms to track the contractile motion of the cardiac tissue and showed that optical mapping can be performed in beating and moving hearts.

\subsubsection{Excitation-contraction coupling}

Excitation-contraction coupling (ECC) is the process by which electrical excitation of the cardiac muscle cell causes its mechanical contraction through the release of calcium $\left(\mathrm{Ca}^{2+}\right)$ stored in the sarcoplasmic reticulum (situated within the cells). The release of $\mathrm{Ca}^{2+}$ from the sarcoplasmic reticulum is triggered by the intake of $\mathrm{Ca}^{2+}$ into the cell as a result of an action potential depolarization. This process is called calcium induced calcium release (CICR) [Cal92] and it is essential for the contraction of cardiomyocytes. $\mathrm{Ca}^{2+}$ released from the sarcoplasmic reticulum binds to a protein called troponin, which is bound to the actin myofilament. Upon binding of $\mathrm{Ca}^{2+}$, troponin un- 


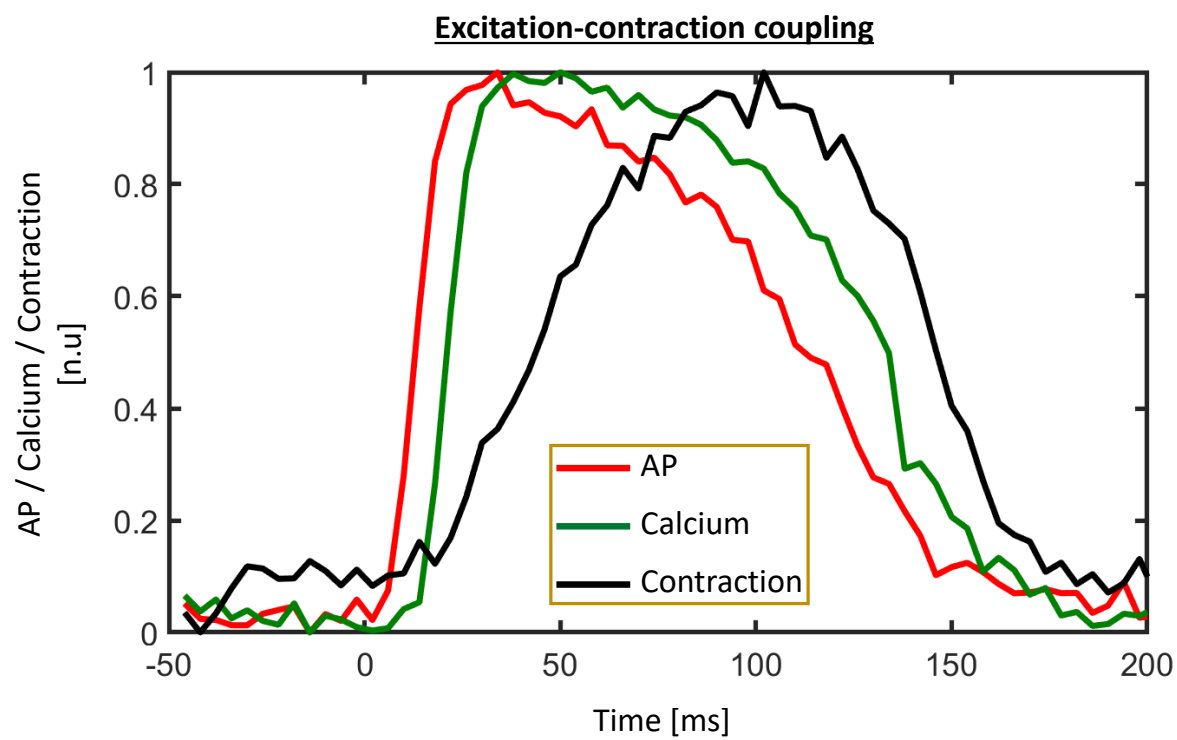

Fig. 2.4: Example of excitation-contraction coupling. Cardiac action potential (AP; red) triggers the increase in intracellular calcium concentration (calcium; green), which finally triggers the mechanical contraction (contraction; black) of the heart muscle. Time series obtained from the left ventricular surface of a rabbit heart during sinus rhythm in an optical mapping experiment. The time traces were spatially averaged from $3 \times 3$ pixels. The heart was loaded with Di-4-ANBDQPQ (voltage sensitive dye) and Rhod-2 AM (calcium sensitive dye) to measure action potential and intracellular calcium concentration simultaneously.

dergoes a conformational change which allows another myofilament, myosin to attach to the actin and causes cell contraction. The mechanism of excitation contraction coupling can be visualized by using optical mapping experiments (see section 2.5) involving beating hearts with the help of voltage and calcium indicators to record action potential, intracellular calcium concentration (increases as a result of CICR) from contracting hearts. An example of excitation-contraction coupling is shown in Figure 2.4. Time traces show cardiac action potential (AP; red), intracellular calcium concentration (calcium; green) and amplitude of contractile motion (contraction; black) obtained with optical mapping (see section 5.4 for motion amplitude calculation details). Action potential causes an increase in intracellular calcium concentration (due to the release of calcium from sarcoplasmic reticulum) and the increase in calcium triggers the mechanical contraction of the muscle by activating the myofilaments. 


\subsubsection{Mechano electric feedback}

Studies in animal hearts show that mechanical perturbations such as stretch of the myocardial tissue can cause electrical depolarization of the tissue. This mechanism is known as mechano electric feedback (MEF) or contraction excitation coupling [Fra96; Sir96]. For example, blows to the chest can alter myocardial stretch or pressure loading prior to or during electrical activation. The MEF together with ECC make the coupling between electrical excitation and mechanical contraction bidirectional. MEF is shown to promote cardiac arrhythmias [Fra96; Sir96]. A rare disease Commotio cordis was observed to cause ventricular fibrillation and sudden cardiac arrest. It is initiated by a localized precordial impact that is not sufficiently strong to cause mechanical damage to the heart or surrounding organs [PT14].

\subsection{Optical mapping of cardiac electrophysiology}

Optical mapping is a fluorescence imaging technique in which voltage sensitive and calcium sensitive fluorescent dyes are used to map action potential and calcium concentration optically from the cardiac tissue. Upon excitation with proper wavelengths, these fluorescent dyes emit fluorescent light which is modulated by the change in membrane potential or by the change in calcium concentration. Since these cardiac activities occur on a millisecond time scale and the change in fluorescent intensity due to them is very small (usually $2-10 \%$ of fractional change in intensity for a change in cell membrane potential of $100 \mathrm{mV}$ ), optical mapping techniques use cameras with high speed and high quantum efficiency.

Potentiometric or voltage sensitive dyes bind to the cardiac cell membrane and shift the wavelengths of absorption or emission with cardiac action potential (see section 3.1.1 for spectral properties of a voltage sensitive dye). The calcium sensitive fluorescent dyes in general show an acute increase or decrease in their fluorescence intensity upon binding of $\mathrm{Ca}^{2+}$. When $\mathrm{Ca}^{2+}$ binds to a ratiometric calcium sensitive dye, it changes the optimum excitation or emission wavelength of the dye [Boo+13]. 


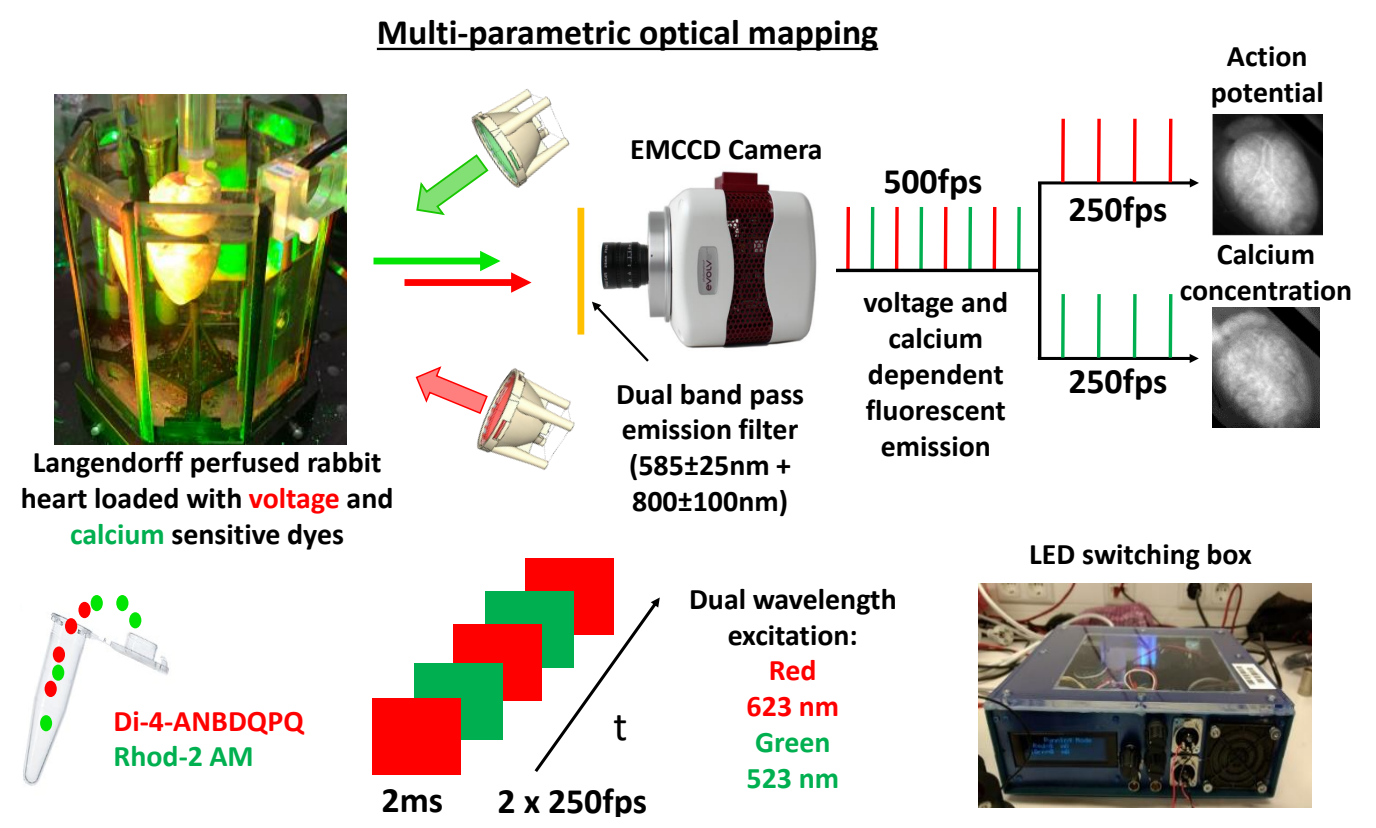

Fig. 2.5: Multi-parametric optical mapping setup illustrating simultaneous imaging of action potential and calcium concentration from a rabbit heart. The rabbit heart kept in a Langendorff perfusion is loaded with voltage sensitive (Di-4-ANBDQPQ) and calcium sensitive (Rhod-2 AM) fluorescent dyes. The fluorescent emission from these dyes shows modulation in intensity due to cardiac action potential and intracellular calcium concentration. The action potential and calcium dependent fluorescent emission are captured by an EMCCD camera equipped with a dual band pass emission filter. The excitation light is switched with each camera frame such that the fluorescent emission captured on the camera can be separated into optical action potential and calcium concentration by using odd-even camera frames.

Figure 2.5 shows an optical mapping setup used to measure cardiac action potential and calcium concentration simultaneously from a Langendorff perfused rabbit heart. The heart is stained with fluorescent dyes which are sensitive to voltage and calcium. Voltage sensitive dye Di-4-ANBDQPQ (red excitation $(620-660 \mathrm{~nm})$ and red emission $(\approx 700 \mathrm{~nm}))$ is used for action potential recordings, whereas calcium sensitive dye Rhod-2 AM (green excitation $(552 \mathrm{~nm}$ ) and yellow emission $(581 \mathrm{~nm}$ ) is used for the recording of calcium concentration. The fluorescent emission containing the information of action potential and calcium concentration are recorded with an EMCCD camera having a dual band pass emission filter $(580 \pm 25 \mathrm{~nm}+800 \pm 100 \mathrm{~nm})$ attached with it. The excitation light is synchronously switched with each camera frame so that odd frames of the camera capture fluorescent intensity 
changes with one excitation wavelength and the even frames record fluorescent changes with the second excitation wavelength. That is, odd frames record fluorescent intensity modulation with action potential/calcium from the cardiac tissue and even frames capture fluorescent intensity modulation with calcium/action potential. The example shown in Figure 2.4 used voltage sensitive dye Di-4-ANBDQPQ for action potential measurement and calcium sensitive dye Rhod-2-AM for calcium concentration measurement.

It is to be noted that, depending on the optics used, optical mapping can obtain high spatial and temporal resolution action potential and calcium concentration signals from isolated hearts kept at physiological conditions. However, this thesis is designed to focus on optical mapping techniques specific to action potential recordings and the measurement of important electrophysiological parameters from Langendorff perfused contracting rabbit hearts.

\subsubsection{Motion artifacts}

The mechanical contraction of the heart is very important for efficient pumping of blood to other organs of the body. Although the above pumping mechanism is absent in Langendorff perfused hearts, the hearts can still contract and cause mechanical deformation and displacement of the tissue. This mechanical contraction can lead to distortions of measured action potential or calcium concentration signals (also in wave propagation) in optical mapping experiments. These distortions due to mechanical motion are generally referred to as motion artifacts.

As illustrated in Figure 2.6 A, the motion artifacts mainly arise due to three reasons:

1. Relative motion between the cardiac tissue and the detector: The contractile motion of the cardiac tissue with respect to the camera results in loss of correspondence between the camera pixel and a particular part of the cardiac tissue. In this case, the electrophysiological signals (optical action potentials) captured by the camera pixel contain the information from more than one location, which causes distortions in the recorded signals.

2. Inhomogeneous illumination: Motion of the cardiac tissue through an inhomogeneous light field contributes to distortion in optical action potential signals. Since the light illumination on the cardiac tissue is not homogeneous, 
the relative motion of the cardiac tissue with respect to the light sources is also an important factor which enhances motion artifacts.

3. Inhomogeneous dye concentration: Loading of voltage/calcium sensitive dyes is not always homogeneous. When the heart contracts, these variations in dye concentration also contribute to the signal distortion due to motion artifacts.

Hence, the overall motion artifact is the combination of all these three factors

A

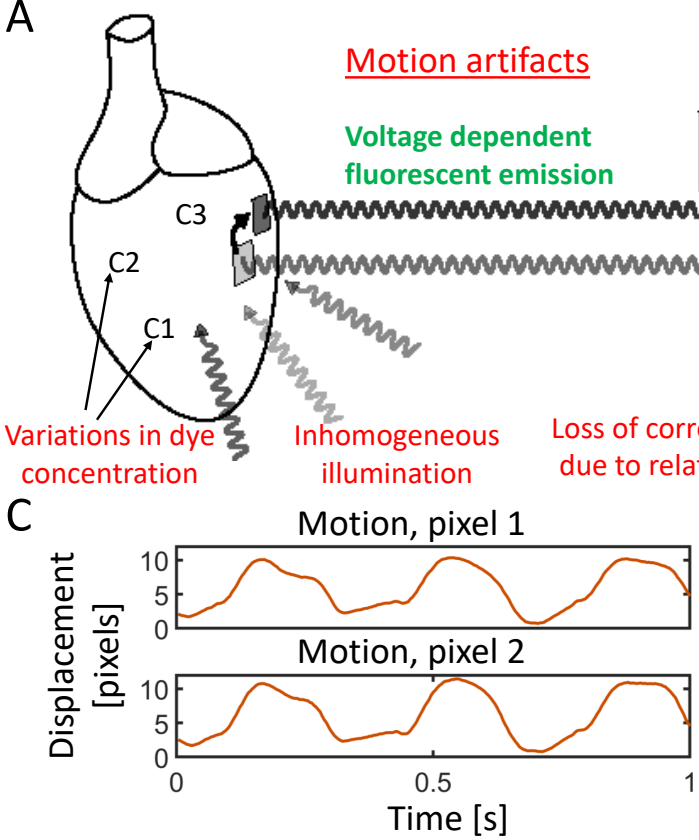

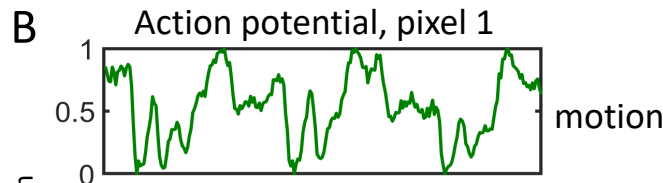

동

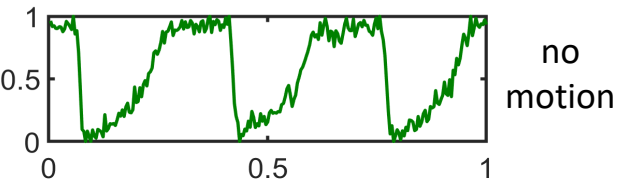

Action potential, pixel 2

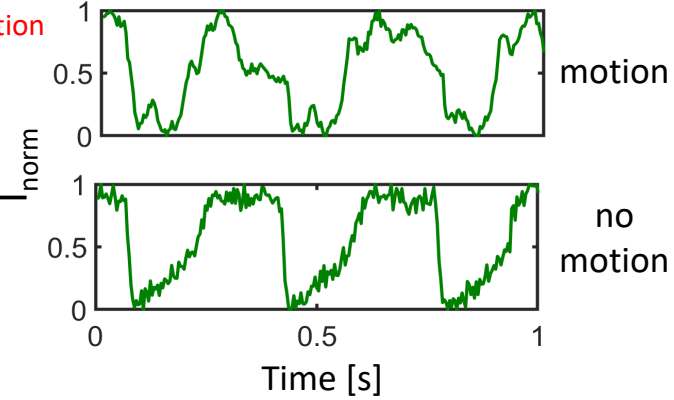

Fig. 2.6: Mechanical contraction of the cardiac tissue causes motion artifacts in optical mapping experiments. A) Sources producing motion artifacts: Loss of correspondence between camera pixel and the tissue due to the relative motion between them, inhomogeneous illumination and spatial (also temporal) dye concentration differences. B) Comparison of action potential time series with and without motion for two different pixels (7 $\mathrm{mm}$ apart) from the left ventricular surface of a rabbit heart. Electromechanical uncoupler Blebbistatin $(5.6 \mu \mathrm{M})$ is used to suppress the motion. The time series with motion are highly distorted due to motion artifacts. C) Motion amplitude (see section 5.4 for computation details) from same pixels used in panel B. Though motion amplitudes are identical in both the pixels (difference in motion amplitude is roughly 1 pixel), the total motion artifacts are different in these pixels as indicated by different signal distortions in panel B (action potential with motion).

and the electrophysiological signals obtained from a contracting heart are usually distorted with motion artifacts arising from all these sources. 
Figure 2.6 B shows the comparison of action potential time traces for two different pixels (pixel 1 and pixel 2 which are $7 \mathrm{~mm}$ apart) from the left ventricular surface of a rabbit heart with (upper row, motion) and without (lower row, no motion) contraction. The upper rows of both pixels show distortions of action potential signals due to motion artifacts resulting from the contractile motion of the heart. The time series in the lower rows are the action potential signals for the same pixels after inhibiting the contraction using Blebbistatin. Figure 2.6 C shows the amplitude of contractile motion, calculated as the displacement of the cardiac tissue (detailed description of the calculation is in section 4.1) for the pixels used in panel B. As it is clear from panel $\mathrm{C}$, the locations of cardiac tissue corresponding to both these pixels displace by similar amounts upon contraction, which results in an identical motion amplitude (the difference in maximum amplitude of motion is around 1 pixel).

Though the pixels displace by virtually identical amounts in panel C, the motion artifacts based signal distortions shown in panel B (upper rows of pixel 1 and 2) are significantly different. This indicates the presence of other factors/sources contributing to the signal distortions such as inhomogeneous lighting and heterogeneity in dye concentration across the heart as indicated in panel A.

\subsubsection{Excitation-contraction uncoupling}

Motion artifact-free electrophysiological signals are of high demand while using optical mapping technique. Motion artifacts emerging from contractile motion have been suppressed pharmacologically using excitation-contraction uncouplers in most of the optical mapping experiments. These uncouplers uncouple the electrical excitation and mechanical contraction by suppressing the mechanical activity of the cardiac tissue whereas, but they preserve the electrical activity of the tissue. Hence these uncouplers enhance the optical mapping signals by significantly reducing motion artifacts. Various uncouplers such as diacetyl monoxime (DAM) [Liu+92], 2,3-butane-dione monoxime (BDM) [Ket+04a; LLE12], Cytochalasin-D [Ket+04a], etc. were used in optical mapping studies to minimize motion artifacts while imaging action potential and calcium concentration from the cardiac tissue. Though all these uncouplers are very effective in reducing the motion artifacts, they 
all have significant limitations. Among these, the most reliable and recent uncoupler is Blebbistatin, which is considered as the electromechanical uncoupler of the choice in most of the optical mapping experiments involving Langendorff perfused hearts, isolated ventricles and atria and ventricular myocytes after its first application in cardiac research [Fed+07]. Blebbistatin inhibits the myofilament myosin and hence, suppresses the mechanical contraction of the hearts. Mechanical constraints were also used in some optical mapping studies to diminish motion related artifacts [Man+98; Lau+98; Che+00]. 


\section{Experimental methods}

This chapter describes the optical mapping experimental techniques used to image action potential wave activity of Langendorff perfused hearts. The voltage sensitive fluorescent dye Di-4-ANEPPS is used to record optical measurements of action potential wave propagation on the surface of isolated rabbit hearts kept at physiological conditions. The chapter starts with an introduction to ratiometric imaging used to enhance action potential signal quality with optical methods by minimizing the adverse effects of several quantities which are independent of action potential. Detailed analysis of excitation and emission spectra of Di-4-ANEPPS and different ratiometric imaging techniques are also described. Finally, the experimental setup including all the important components used for action potential measurements is introduced and a short overview of optical mapping data processing is given.

\subsection{Ratiometry}

A drawback of the conventional optical mapping method (Figure 2.5) is that the fluorescence signal captured by the detector from a particular location contains not only the information of electrophysiological parameters of interest but also the local effective dye concentration and local excitation light intensity. The effective dye concentration and excitation light intensity are likely to vary spatially across the cardiac tissue. Additionally, there are temporal variations of dye concentration due to photobleaching, washout and compartmentalization $[\mathrm{Bac}+11]$. These inhomogeneities in dye concentration and excitation light intensity produce different fractional changes in fluorescence $(\Delta \mathrm{F})$ with respect to the electrophysiological parameter under study, in different regions of the heart. Hence, the nonuniformity in dye concentration and light intensity not only make the direct comparison of electrophysiological parameters within the heart difficult, but also contributes to 
total motion artifacts as discussed in section 2.5.1 when optical mapping is performed with contracting hearts.

The ratiometric optical mapping technique minimizes the effects of inhomogeneous dye loading and variation in light intensities by using common mode rejection technique [Kni+00; Bac+11]. Ratiometry uses two bands of wavelengths for excitation or emission unlike conventional optical mapping, where single excitation and emission bands are used. As the name indicates, ratiometry is a measurement involving the ratio of two fluorescence signals, which have different fractional changes of fluorescence $(\Delta \mathrm{F})$ with respect to the electrophysiological parameters of interest, but have common artifacts from all other sources (dye loading and lighting inhomogeneities). Hence taking the ratio between these two fluorescence signals cancels out the artifacts common to both the signals and improves the signal quality.

Two commonly used ratiometric techniques are emission and excitation ratiometry. Emission ratiometry uses single excitation wavelength and two emission wavelengths are recorded with two detectors, which are aligned to image the same location of the cardiac tissue (corresponding pixels of both detectors image the same tissue). Emission ratiometry was used to reduce motion artifacts in rabbit hearts with two photomultiplier tubes [Kni+00] to collect the fluorescent emission. Though emission ratiometry is a useful tool in obtaining high resolution electrophysiological signals with minimal motion artifacts (artifacts due to illumination and dye concentration), the complexity in aligning the detectors and the cost of the detectors are the main drawbacks of this technique.

Excitation ratiometry uses two excitation wavelengths and a single emission band to record the fluorescence signals. The excitation wavelengths are usually switched with high frequency and the fluorescence signals acquired with a single emission band are used for ratiometric measurement. Excitation ratiometry is simpler than emission ratiometry since there is no need to align two detectors carefully to perform ratiometric measurements on electrophysiological signals. But the excitation light needs to be switched with high frequency to record the fast variations in signals with both excitation wavelengths. Nowadays, this is achieved through high power LEDs, which can be switched at higher speeds compared to the traditional light sources. Excitation ratiometry was used to measure cardiac action potentials with reduced motion artifacts $[\mathrm{Bac}+11]$. It was also combined with marker based 
motion tracking to record action potential signals from contracting hearts [Zha+16].

To minimize the dye loading and the illumination (partially) artifacts from the optical action potential signals, excitation ratiometry is preferred over emission ratiometry in this thesis because of two main reasons. First, as mentioned before, due to the complexity in the alignment of two cameras to image same location of the cardiac tissue. Second due to the ease of availability of components for excitation ratiometry. For example, an LED switching box (same as shown in Figure 2.5) and optical components (optical filters, LEDs and lenses) for one excitation wavelength were already available in the lab from previous experiments (multi-parametric imaging).

\subsubsection{Excitation ratiometry}

Excitation ratiometry utilizes the ratiometric properties of the voltage sensitive fluorescent dye Di-4-ANEPPS. This dye undergoes voltage dependent spectral shifts in the absorption and emission spectra as shown in Figure 3.1 during electrical excitation of the cardiac tissue with the action potential. As shown in Figure 3.1 A, the absorption spectrum of Di-4-ANEPPS show spectral shifts towards lower wavelengths for all the excitation wavelengths within the range $360 \mathrm{~nm}-575 \mathrm{~nm}$. As a result, the excitation wavelengths above the excitation isosbestic point (wavelength at which no change in absorption between resting and depolarization $\approx 480 \mathrm{~nm}$ ) show opposite polarities in voltage dependent spectral change compared to the wavelengths below the isosbestic point. These voltage dependent excitation shifts produce voltage dependent shifts in emission spectra as shown in Figure 3.1 B. The black circles show the emission isosbestic points, where the resting and depolarized cardiac tissue produce no change in fluorescence emission. The emission spectra for blue and cyan excitation undergo action potential dependent fractional change in fluorescence $\Delta \mathrm{F}$ in opposite directions. $\Delta \mathrm{F}$ is positive for blue excitation whereas, $\Delta \mathrm{F}$ is negative with cyan excitation wavelength.

Since the cyan and green excitation light has comparable wavelengths and the fluorescent emission for green excitation shows negative value of $\Delta \mathrm{F}$ similar to cyan excitation wavelength, it is reasonable to perform ratiometric measurements with green excitation light instead of cyan provided there 


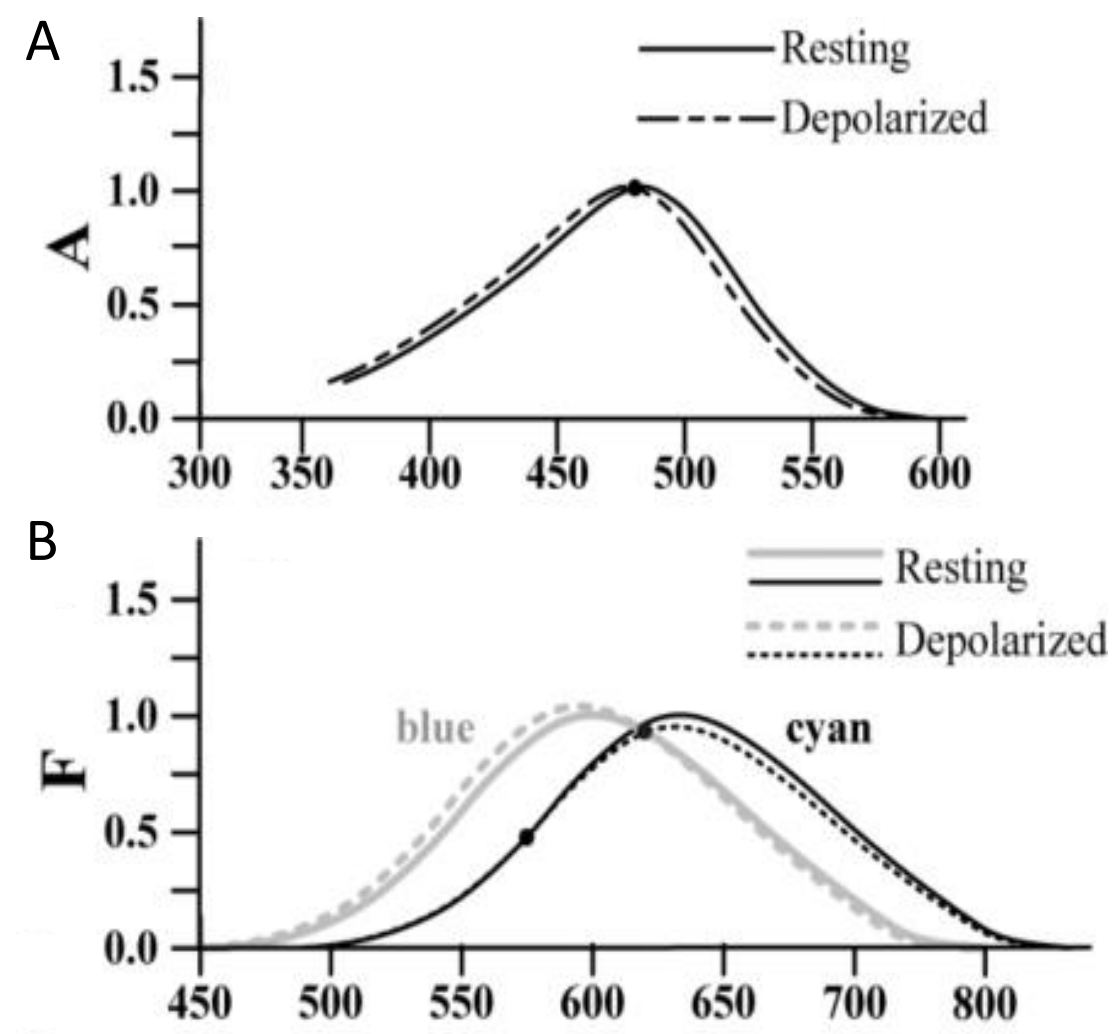

Fig. 3.1: Action potential dependent absorption and emission spectra of Di-4ANEPPS. A) Voltage dependent shift in excitation spectra from resting state (solid) to depolarized state (dashed) of the cardiac tissue with an action potential upstroke. Black circle represents the isosbestic wavelength at which no change in excitation between resting and depolarized states. B) Voltage dependent shift in emission spectra for blue $(450 \mathrm{~nm})$ and cyan $(505 \mathrm{~nm}$ ) excitation wavelengths. Blue and cyan excitation result in two resting emission spectra (solid gray and black lines, respectively). The emission spectra undergo a voltage dependent shift and amplitude modulation resulting from the action potential-dependent excitation shift (dashed lines). The black circles show the emission isosbestic points at which there is no change in fluorescent emission between resting and depolarized states. Note that, blue and cyan excitation wavelengths produce fractional change in fluorescence with opposite polarities. Figure reused with permission from $[\mathrm{Bac}+11]$. 
exists no signal cross talk. Hence, the excitation ratiometric measurements in this thesis were performed by utilizing blue and green excitation wavelengths. The action potential modulated fluorescent emissions were recorded on a single EMCCD camera with the help of a bandpass emission filter (590 $\mathrm{nm}$ $\pm 55 \mathrm{~nm}$ ).

Though blue and green elicited action potentials contain $\Delta \mathrm{F}$ of different magnitudes and polarities, the effective dye concentration on the cardiac tissue is the same for both wavelengths. Also, it is possible to make illumination intensity nearly homogeneous by adjusting blue and green excitation light during the experiment. Hence, the ratio of blue and green elicited action potential signals is less susceptible to variation in dye concentration and illumination intensity across the heart tissue. Additionally, the opposing nature of $\Delta \mathrm{F}$ polarity amplifies the action potential sensitivity while taking the ratio between these two signals.

\subsection{Experimental setup and description}

Figure 3.2 shows the schematic of the ratiometric optical mapping (excitation ratiometry) experiment used to record action potential signals from rabbit hearts kept at physiological conditions. The excitation ratiometric imaging system uses two wavelengths of excitation (blue and green) and collects the fluorescent emission on a single detector. Blue and green excitation light were provided by two sets of high-power light-emitting diodes (3 blue LEDs: $460 \mathrm{~nm}$ center wavelength, model LZ4-40B208; 3 green LEDs: 523nm center wavelength, model LZ4-40G108, both by LED Engin Inc., USA). The blue and green excitation light was further filtered by two sets of narrow bandpass filters mounted in front of the light-emitting diodes (blue: $460 \mathrm{~nm} \pm 5 \mathrm{~nm}$, Thorlabs, USA; green: $540 \mathrm{~nm} \pm 12.5 \mathrm{~nm}$, Chroma Technology Corp., USA). The excitation light was also collimated with plano convex lenses (LA1951-A, Thorlabs, USA), which were placed between the LEDs and the excitation filters. The action potential modulated fluorescent light from the cardiac tissue for blue and green excitation are captured with an EMCCD camera (Evolve 128, Photometrics 128x128 pixels, 16-bit dynamic range) equipped with a lens $(0.95 / 25 \mathrm{~mm}$, Navitar) and a bandpass emission filter (590 $\mathrm{nm}$ $\pm 55 \mathrm{~nm}$, Omega Optical). An LED switching box (from I. Uzelac and F. Fenton, Georgia Tech) switches the light between blue and green with a 
A

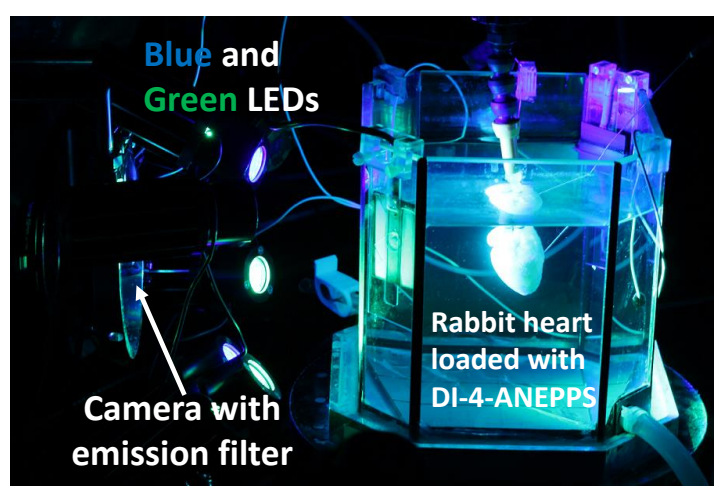

C

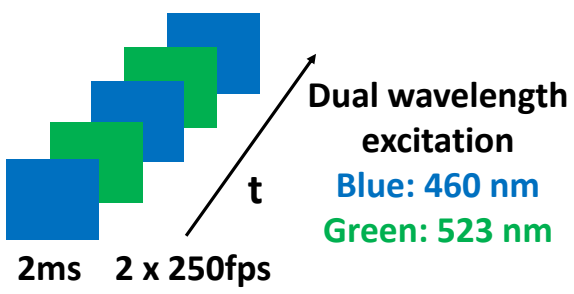

B
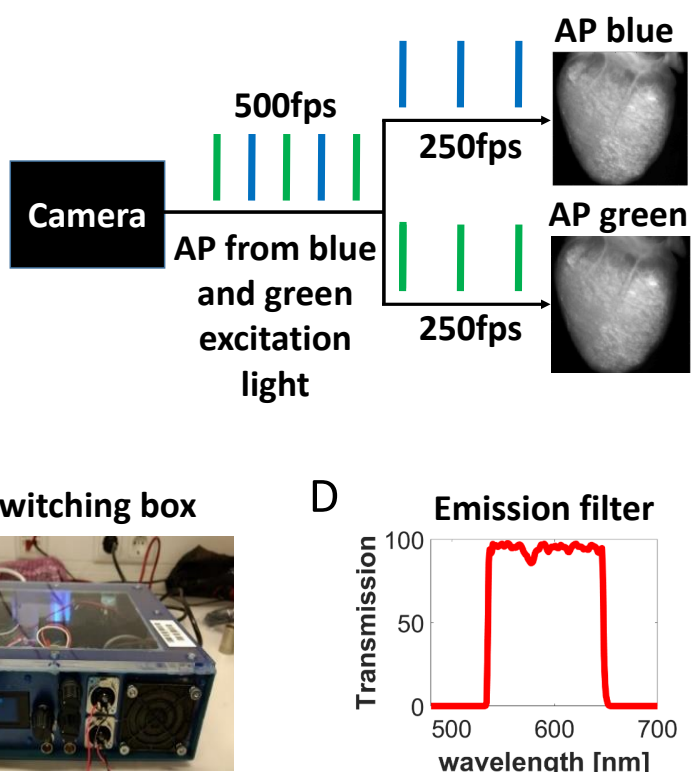

Fig. 3.2: Experimental setup showing ratiometric optical mapping. A) Langendorff perfused contracting rabbit heart is loaded with voltage sensitive dye Di4-ANEPPS. Emitted fluorescent light from the cardiac tissue, modulated by the action potential for blue and green excitation is captured by an EMCCD camera equipped with a bandpass emission filter. B) Separating the camera frames (500 fps) into odd and even frames yields the action potential for blue and green excitation (250 fps in each channel). C) Dual wavelength excitation used for ratiometric imaging. A light switching led driver box is used to switch the excitation light between blue and green(light pulses are of $1.85 \mathrm{~ms}$ duration) with each camera frame. D) A bandpass emission filter $(590 \mathrm{~nm} \pm 55 \mathrm{~nm}$ ) is used to capture action potential corresponding to blue and green excitation with a single camera. 
high frequency $(500 \mathrm{~Hz}$ ) and this switching is synchronized with the camera (records at 500 frames per second) using a camera trigger output from the LED switching box.

\subsection{Optical mapping data processing}

Multiple pre-processing steps have been performed on optical mapping data to precisely calculate electrophysiological parameters of interest from contracting and Blebbistatin-uncoupled hearts. The optical mapping setup shown in Figure 3.2 records action potential signals corresponding to blue and green excitation wavelengths interlaced in time. The first step of data preprocessing is the separation of these interlaced time traces into action potentials corresponding to blue and green excitation light. The camera frames containing action potential modulated fluorescent intensities $(F)$ for blue and green excitation light are separated into odd and even video frames such that all odd frames represent action potential due to blue excitation, whereas all even frames correspond to action potential due to green excitation light. The Figure 3.3 illustrates the splitting of time interlaced optical action potentials (contain action potentials due to both blue and green excitation light; 2000 frames) into action potentials corresponding to blue and green excitation light (1000 frames each). The time traces represent optical action potentials from a single pixel corresponding to a portion of left ventricular tissue. Panel A shows the time interlaced action potentials from a beating heart (raw signal) of 4 seconds duration with 500 fps. Panel B illustrates the separation of time traces shown in panel A into action potentials corresponding to blue and green excitation light (each of 4 seconds duration with 250 fps). Panel C shows the time interlaced action potentials in Blebbistatin-uncoupled condition. Panel D illustrates the separation of time interlaced action potentials shown in panel $\mathrm{C}$ into action potentials of blue and green excitation light.

Action potential modulated fluorescent video sequences obtained with blue and green excitation light are stored as normalized videos by normalizing the fluorescent intensity values of the video sequences using the minimum and maximum intensity values of the entire fluorescent video $f v$ as

$$
I_{n o r m}(x, y, t)=(f v(x, y, t)-\min (f v)) /(\max (f v)-\min (f v))
$$


A
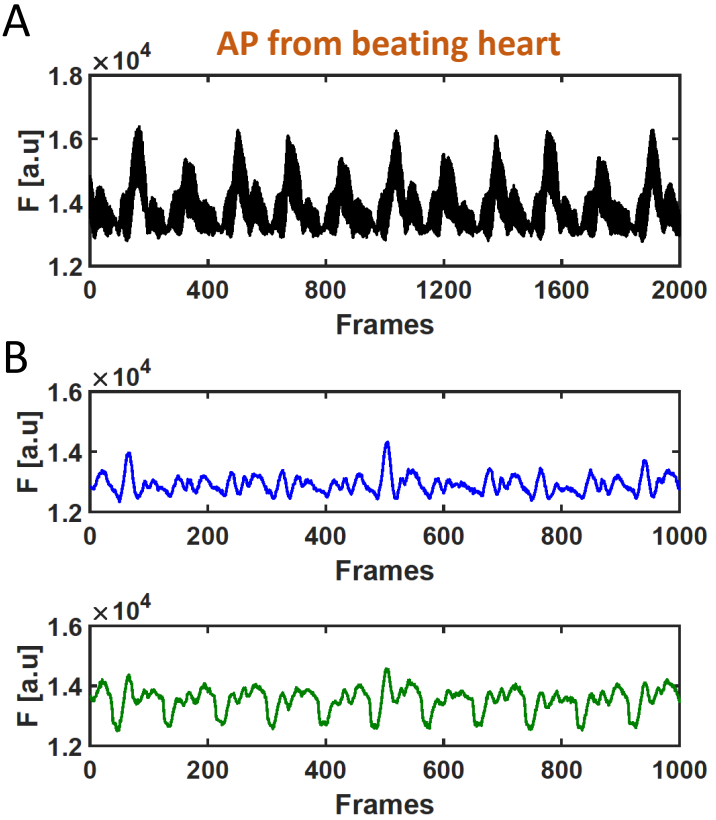

C
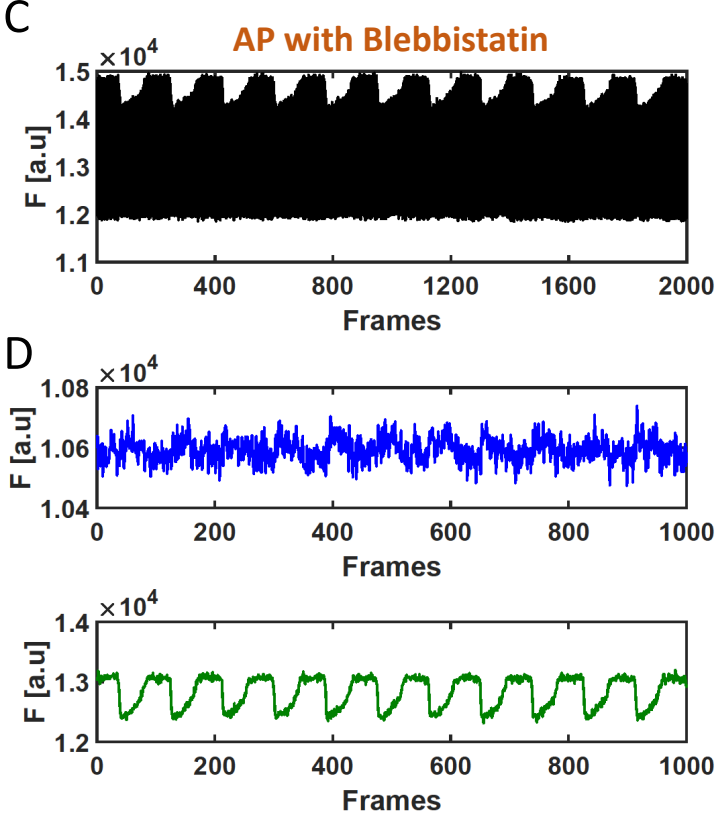

Fig. 3.3: Exemplary time traces (relative fluorescent intensity $F$ as a function of time or frames) from single pixel (from left ventricle) illustrating optical mapping data pre-processing. A) Time interlaced optical action potentials due to blue and green excitation light in contracting condition. B) Separation of time traces shown in panel A into action potentials corresponding to blue and green excitation. C) Time interlaced optical action potentials in Blebbistatin-uncoupled condition. D) Separation of time series shown in panel C into action potentials corresponding to blue and green excitation light.

The normalized fluorescent videos are used for further data processing. Numerical motion tracking techniques (see section 4.1) are used to track the motion of the heart from normalized fluorescent videos of blue and green excitation light, separately. The ratio between motion tracked fluorescent videos of blue and green excitation light are computed and stored for ratiometric measurements. Similarly, ratio video sequences are computed and stored from action potentials corresponding to blue and green excitation in Blebbistatin-uncoupled condition as well.

\subsubsection{Spatio-temporal smoothing}

For the precise measurement of electrophysiological parameters such as action potential duration, ratio video images are smoothed spatio-temporally. It means the intensity value of each pixel is averaged spatially using surround- 
ing pixels and temporally using nearby frames according to size of the kernel. The data processing mentioned in this thesis used spatio-tempral filtering with a kernel size of $3 \times 3 \times 3$. In short it means $3 \times 3$ pixels and 3 frames.

A

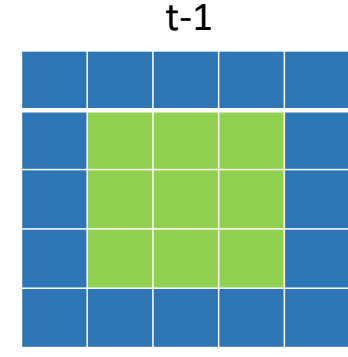

B

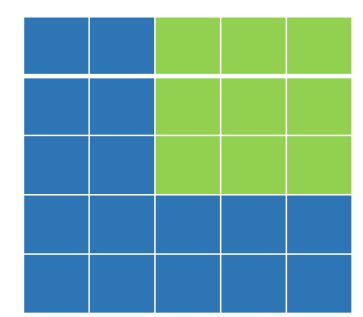

$\mathrm{t}$
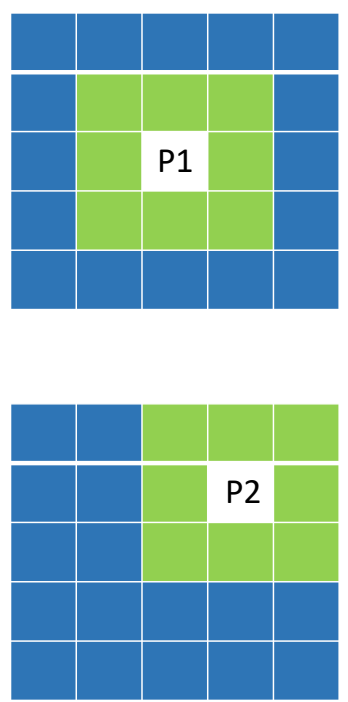

$t+1$
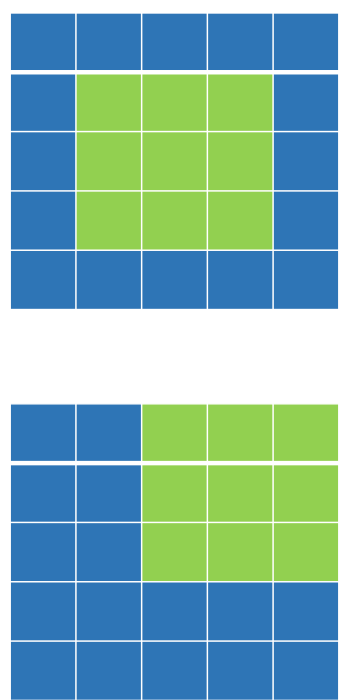

Fig. 3.4: Concept of spatio-temporal smoothing with a kernel size of $3 \times 3 \times 3$ for two different pixels $\mathrm{P}_{1}$ and $\mathrm{P}_{2}$. The intensity value of $\mathrm{P}_{1}$ (or $\mathrm{P}_{2}$ ) in the smoothed video is calculated as the mean value of fluorescent intensities from the neighbouring $3 \times 3$ pixels (green) including $\mathrm{P}_{1}$ (or $\mathrm{P}_{2}$ ) from frames $t, t-1$ and $t+1$.

Figure 3.4 illustrates the idea of spatio-temporal smoothing for two different pixels $\mathrm{P}_{1}$ and $\mathrm{P}_{2}$. To compute the smoothed intensity value corresponding to the pixel $\mathrm{P}_{1}$ at frame $t$, the intensity values of $3 \times 3$ neighbouring pixels with $\mathrm{P}_{1}$ as the center is constructed. Similarly corresponding $3 \times 3$ pixels are identified in frames $t-1$ and $t+1$. The intensity value of $P_{1}$ in the smoothed video is calculated as the mean value of intensities from all these 27 pixels. The complete smoothed video is produced by repeating the method for all pixels and for all the video frames. For measurement of electrophysiological parameters such as action potential duration and frequency of ventricular fibrillation, spatio-temporal smoothing with a kernel size of $3 \times 3 \times 3$ is used consistently in this thesis. This filtering was used to measure the desired quantities from all pixels in the video images (signal to noise ratio gets worse at some pixels). However, for visualization of the quantities spatial averaging of $3 \times 3$ pixels is often used. 


\subsubsection{Sliding window normalization}

The automatic calculation of action potential durations (see section 5.1.1) is much easier when all the action potentials of a particular pixel (after smoothing) are at same height for all the frames. To do this, action potentials

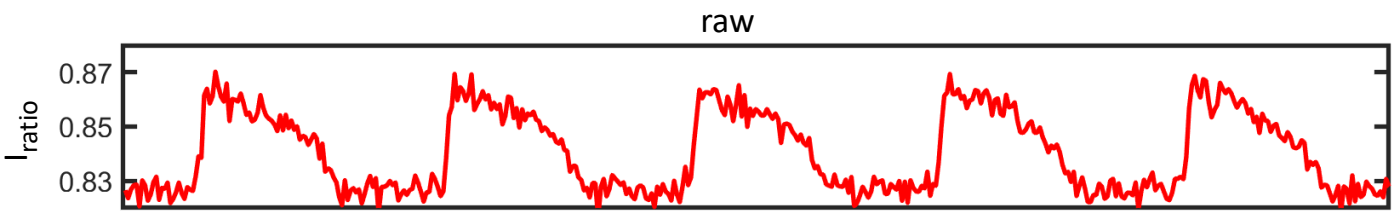

smoothed

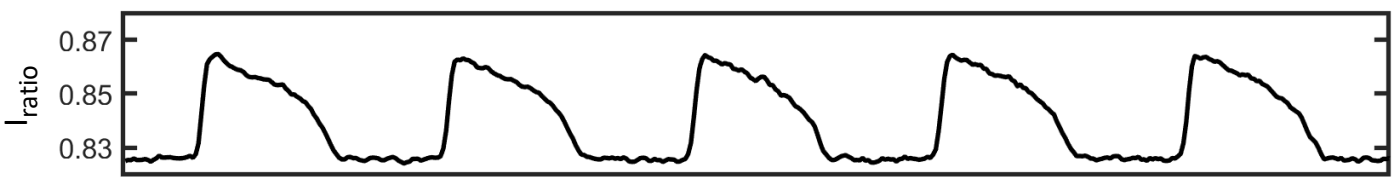

window normalization

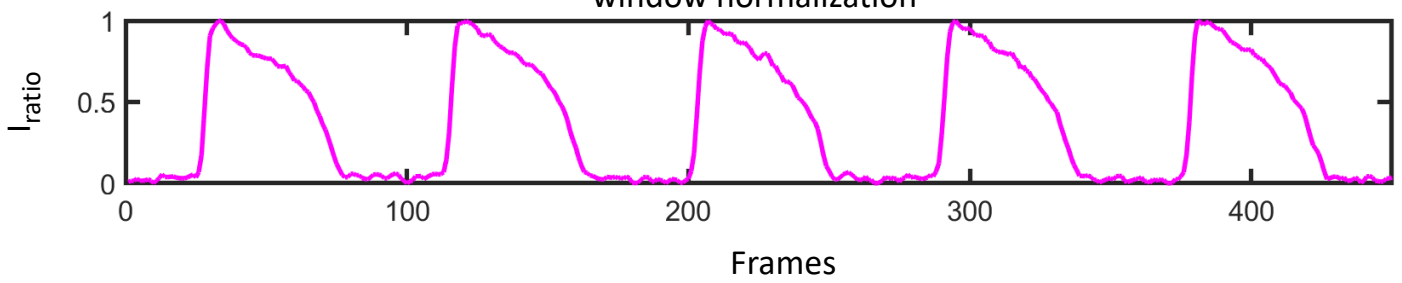

Fig. 3.5: Exemplary time traces illustrating the effect of smoothing and normalization of optical action potentials. The smoothed optical action potential time traces (smoothed; black) are obtained by spatio-temporal averaging $(3 \times 3 \times 3)$ of single pixel time traces (raw; red). A sliding window normalization is used to normalize each action potential (window normalization; magenta) in the interval [0 1]. The time traces are obtained from the left ventricular surface of a rabbit heart during electrical pacing with $C L=350$ $m s$. The contraction of the heart was inhibited by Blebbistatin.

are extracted for each pixel and all the action potentials are normalized in the range [0 1] using a sliding window normalization. A window of proper size is moved along the time axis and the minimum and maximum values of action potentials in every time window are calculated. The action potentials are normalized within that window using the observed minimum and maximum values. Each action potential is normalized by choosing a window size equal to the number of frames corresponding to a single action potential. The Matlab commands movmin and movmax are used to calculate minimum and maximum values of action potential within each time window. 
Figure 3.5 shows the effects of spatio-temporal smoothing and sliding window normalization on single pixel optical action potential time traces. The time traces are calculated from ratio video images in Blebbistain-uncoupled condition. The spatio-temporal smoothing of single pixel time traces (raw; red) resulted in smoothed action potentials (smoothed; black) with less noise in it. The smoothed action potential traces are normalized with a sliding window normalization (window normalization; magenta) with a proper window size ( $\approx$ number of frames corresponding to one action potential, $w=100$ here). 



\section{Motion artifacts reduction techniques}

As discussed in section 2.5.1, the fluorescence signal distortion due to motion artifacts is a limitation in optical mapping experiments. These motion artifacts of electrophysiological signals are usually removed as described in section 2.5.2 either by using excitation-contraction uncouplers or by mechanically restricting the heart. The electromechanical uncouplers are effectively reducing motion artifacts in action potential or calcium concentration signals. However, some of these uncouplers were shown to have effect on cardiac electrophysiology. Additionally, uncoupling of electrical activity from cardiac mechanics is not physiological since there is no mechanical contraction involved. On the other hand, numerical motion tracking was performed to record action potential and mechanical contraction in Langendorff perfused contracting hearts by attaching polyethylene markers on the left ventricular epicardium [Bou+11; Zha+16]. Though these studies were able to image action potential wave propagation from beating hearts without using pharmacological uncoupling, the presence of markers reduces the spatial resolution of obtained fluorescence signals. And, recently, marker-free motion tracking was used to numerically track and compensate the motion of contracting hearts to measure action potential wave phenomena propagating across the contracting heart surface [CL18]. This chapter describes the marker-free numerical motion tracking technique used to measure action potential signals from optical mapping studies involving beating hearts.

\subsection{Marker-free motion tracking}

The basic concept behind motion tracking is to find geometric transformations that align (or register) multiple images with a reference image. In order to obtain action potential signals from a contracting heart (with minimum 
motion artifacts), the contractile motion of the heart has to be tracked for all camera frames with respect to a reference frame. This section describes the motion tracking algorithm used to track and register video sequences of a contracting heart. The motion tracking algorithm is based on the work by Periaswamy et al. [PF03; KP03].

Let $f(x, y, t)$ and $f(\hat{x}, \hat{y}, t-1)$ represent test and reference images respectively. By assuming brightness constancy (difference in brightness between these images is only due to motion and not due to any brightness variations) and a local affine model for transformation between the images results in

$$
f(x, y, t)=f\left(a_{1} x+a_{2} y+a_{5}, a_{3} x+a_{4} y+a_{6}, t-1\right)
$$

where $a_{1}, a_{2}, a_{3}, a_{4}$ form the affine matrix $A$ and $a_{5}, a_{6}$ form the displacement vector $\vec{d}$ :

$$
A=\left[\begin{array}{ll}
a_{1} & a_{2} \\
a_{3} & a_{4}
\end{array}\right]
$$

and

$$
\vec{d}=\left[\begin{array}{l}
a_{5} \\
a_{6}
\end{array}\right]
$$

Now, the goal is to compute the six parameters,

$$
\vec{a}=\left(\begin{array}{llllll}
a_{1} & a_{2} & a_{3} & a_{4} & a_{5} & a_{6}
\end{array}\right)^{T}
$$

corresponding to the geometrical transformation between test and reference images. These parameters are calculated by minimizing the following quadratic error function,

$$
E(\vec{a})=\sum_{x, y \in \Omega}\left[f(x, y, t)-f\left(a_{1} x+a_{2} y+a_{5}, a_{3} x+a_{4} y+a_{6}, t-1\right)\right]^{2}
$$

such that test image aligns with the reference image when the error is at minimum value. $\Omega$ in (4.3) represents a small spatial neighborhood.

Since the functional form of $f$ on the parameters $\left(a_{i}\right)$ are unknown, the error function in (4.3) cannot be minimized analytically. Hence, the minimization is simplified by approximating the error function using a first-order truncated Taylor series expansion: 


$$
\begin{array}{r}
E(\vec{a}) \approx \sum_{x, y \in \Omega}\left(f(x, y, t)-\left[f(x, y, t)+\left(a_{1} x+a_{2} y+a_{5}-x\right) f_{x}(x, y, t)+\right.\right. \\
\left.\left.\left(a_{3} x+a_{4} y+a_{6}-y\right) f_{y}(x, y, t)-f_{t}(x, y, t)\right]\right)^{2}
\end{array}
$$

where $f_{x}(),. f_{y}($.$) are spatial derivatives of f($.$) and f_{t}($.$) is the temporal$ derivative of $f($.$) . The error function in (4.4) further reduces to$

$$
\begin{array}{r}
E(\vec{a})=\sum_{x, y \in \Omega}\left[f_{t}(x, y, t)-\left(a_{1} x+a_{2} y+a_{5}-x\right) f_{x}(x, y, t)-\right. \\
\left.\left(a_{3} x+a_{4} y+a_{6}-y\right) f_{y}(x, y, t)\right]^{2}
\end{array}
$$

The error function is expressed more compactly in vector form as:

$$
E(\vec{a})=\sum_{x, y \in \Omega}\left(k-\vec{c}^{T} \vec{a}\right)^{2}
$$

where the vector $\vec{c}$ and scalar $k$ are defined as:

$$
\begin{gathered}
k=f_{t}+x f_{x}+y f_{y} \\
\vec{c}=\left(\begin{array}{llllll}
x f_{x} & y f_{x} & x f_{y} & y f_{y} & f_{x} & f_{y}
\end{array}\right)^{T} ;
\end{gathered}
$$

Now, the error function in (4.6) can be minimized analytically by differentiating with respect to $\vec{a}$ :

$$
\frac{d E}{d \vec{a}}=\sum_{x, y \in \Omega}-2 \vec{c}\left(k-\vec{c}^{T} \vec{a}\right)
$$

setting equal to zero and solving for $\vec{a}$

$$
\vec{a}=\left(\sum_{x, y \in \Omega} \vec{c} \vec{c}^{T}\right)^{-1}\left(\sum_{x, y \in \Omega} \vec{c} k\right)
$$


This solution assumes that the $6 \times 6$ matrix $\left(\sum_{x, y \in \Omega} \vec{c} \vec{c}^{T}\right)$ is invertible. This can usually be guaranteed by integrating over a large enough spatial region $\Omega$ with sufficient image content.

The algorithm discussed above uses brightness constancy assumption. However this assumption fails when the fluorescent intensity recorded on the camera changes due cardiac action potentials. For example, an action potential upstroke results in 4\% fractional change in fluorescent intensity (with Di-4-ANEPPS) within 8-12 ms (2-3 camera frames) duration.

Hence, to account for intensity variations, an explicit change of local contrast and brightness is incorporated into (4.11) as

$$
a_{7} f(x, y, t)+a_{8}=f\left(a_{1} x+a_{2} y+a_{5}, a_{3} x+a_{4} y+a_{6}, t-1\right)
$$

where $a_{7}$ and $a_{8}$ are spatially varying parameters that incorporate changes in contrast and brightness, respectively. The error function in this case is approximated using Taylor series expansion as before to produce:

$$
E_{i}\left(\overrightarrow{a_{i}}\right)=\sum_{x, y \in \Omega}\left(k_{i}-\vec{c}_{i}^{T} \vec{a}_{i}\right)^{2}
$$

Where

$$
\begin{gathered}
k_{i}=f_{t}-f+x f_{x}+y f_{y} \\
\overrightarrow{c_{i}}=\left(\begin{array}{llllllll}
x f_{x} & y f_{x} & x f_{y} & y f_{y} & f_{x} & f_{y} & -f & -1
\end{array}\right)^{T}
\end{gathered}
$$

Minimization of (4.12) yields:

$$
\overrightarrow{a_{i}}=\left(\sum_{x, y \in \Omega} \overrightarrow{c_{i}}{\overrightarrow{c_{i}}}^{T}\right)^{-1}\left(\sum_{x, y \in \Omega} \overrightarrow{c_{i}} k_{i}\right)
$$

(4.15) is used to compute local affine and contrast/brightness parameters within a small spatial neighbourhood $\Omega$. The corresponding model parameters $\left(\overrightarrow{a_{i}}\right)$ are assumed to be constant in this spatial neighborhood. Choosing the size of $\Omega$ is tricky due to to the fact that selecting larger area makes it more likely that $\sum_{x, y \in \Omega} \overrightarrow{c_{i}}{\overrightarrow{c_{i}}}^{T}$ will be invertible. On the other hand, a smaller spatial neighborhood makes it more likely that brightness constancy assumption will hold. The balancing of these two issues is important for accurate calculation of model parameters. Hence, to avoid the difficulty in 
balancing these issues, the constancy assumption of model parameters are replaced by smoothness constraint assumption in which the local affine and contrast/brightness parameters vary smoothly across space.

The smoothness constraint is incorporated into the algorithm by augmenting the error function shown in equation (4.12) with an error function corresponding to smoothness constraint $E_{s}\left(\overrightarrow{a_{i}}\right)$ as

$$
E_{b s}\left(\overrightarrow{a_{i}}\right)=E_{b}\left(\overrightarrow{a_{i}}\right)+E_{s}\left(\overrightarrow{a_{i}}\right)
$$

where $E_{b}\left(\overrightarrow{a_{i}}\right)$ is same as equation (4.12) without summation.

$$
E_{b}\left(\overrightarrow{a_{i}}\right)=\left[k_{i}-{\overrightarrow{c_{i}}}^{T}{\overrightarrow{a_{i}}}^{2}\right.
$$

and

$$
E_{s}\left(\overrightarrow{a_{i}}\right)=\sum_{i=1}^{8} \lambda_{i}\left[\left(\frac{\partial a_{i}}{\partial x}\right)^{2}+\left(\frac{\partial a_{i}}{\partial y}\right)^{2}\right]
$$

where $\lambda_{i}$ is a positive constant that controls the relative weight given to the smoothness constraint on parameter $a_{i}$. As before, the error function is minimized by differentiating with respect to $\overrightarrow{a_{i}}$. Differentiation yields:

$$
\frac{d E_{b}}{d \overrightarrow{a_{i}}}=-2 \vec{c}\left(k_{i}-{\overrightarrow{c_{i}}}^{T}{\overrightarrow{a_{i}}}\right)
$$

and

$$
\frac{d E_{s}}{d \overrightarrow{a_{i}}}=2 L\left(a_{m}-\overrightarrow{a_{i}}\right)
$$

Where $\mathrm{L}$ is an $8 \times 8$ diagonal matrix with diagonal elements $\lambda_{i}$ and zero off diagonal elements. $a_{m}$ is the component-wise average of $\overrightarrow{a_{i}}$ over a spatial neighborhood.

An iterative relation is obtained for the calculation of model parameters by setting the sum of above two differentials to zero. The parameters $a_{i}$ are calculated iteratively as given below:

$$
a_{i}{ }^{(\mathrm{j}+1)}=\left(\overrightarrow{c_{i}}{\overrightarrow{c_{i}}}^{T}+L\right)^{-1}\left(\overrightarrow{c_{i}} k_{i}+L a_{m}^{\mathrm{j}}\right)
$$

$a_{m}(\mathrm{j})$ on each iteration $j$ is calculated from the current $a_{i}(\mathrm{j})$.

On each iteration, the geometric transformation estimated from equation (4.21) is applied to the test image and a new transformation is estimated between the reference image and the newly warped test image. Additionally, 
to deal with larger motions, a course to fine scheme is included in the algorithm. Initially, the geometric transformation is estimated at the coarsest level using a Gaussian pyramid for both test and reference images. The geometric transformation estimated in the coarsest level is used to warp the test image in the next level of the pyramid. A new estimate of the transformation is computed at this level, and the process is repeated at each level of the pyramid. The transformations at each level of the pyramid are accumulated yielding a single final geometric transformation between the test and reference images.

Figure 4.1 shows an example of motion tracking on a fluorescent video image of a contracting heart obtained with optical mapping experiment. Panel A shows the variation of the amplitude of displacements across the pixels. Panel B illustrates the direction of contractile motion from two areas

A

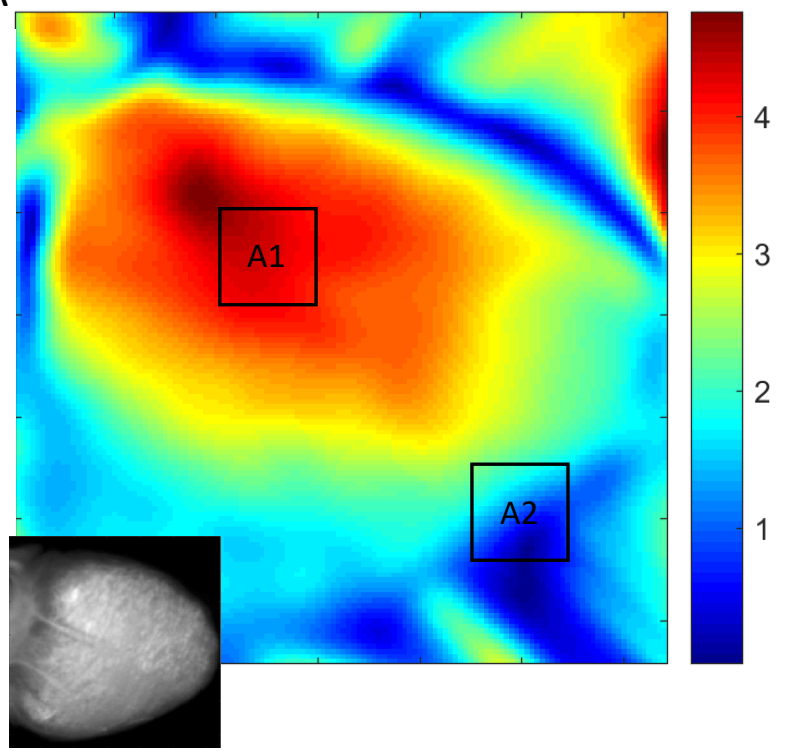

B

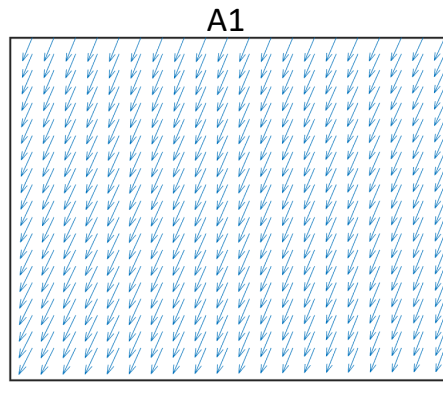

$\mathrm{A} 2$

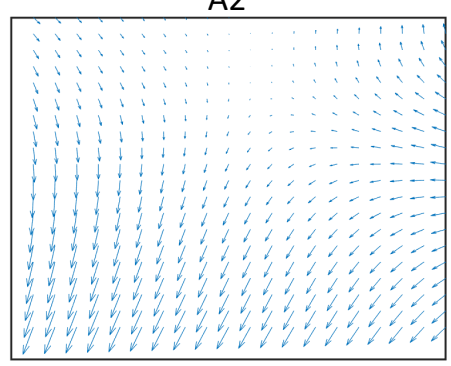

Fig. 4.1: Example of motion tracking showing amplitude and direction of tissue displacement calculated from the action potential video images obtained with optical mapping. The displacement vectors were obtained between two video images which were $300 \mathrm{~ms}$ apart in time. A) Magnitude of tissue displacement in pixels. Bottom left image represents raw test image $(128 \times 128$ pixels, physical size of $30 \mathrm{~mm} \times 20 \mathrm{~mm})$. B) Displacement vectors illustrating the direction of tissue movement upon contraction from two different areas $A 1$ and $A 2$ of the heart. Area $A 1$ shows uniform motion of the cardiac tissue whereas, the area including the boundary of the heart $A 2$ shows non-uniform distribution of the displacement vectors, indicating a limitation of the motion tracking algorithm at the boundary. 
(A1 and A2) on the left ventricular surface. The displacement vectors from area $A 1$ illustrate uniform motion of the cardiac tissue. But the displacement vectors from area $A 2$ show non-uniform motion of the heart tissue due to the limitation of motion tracking algorithm in calculating the model parameters at the boundaries of the heart.

\subsubsection{Motion compensation}

Motion compensated videos were obtained from the original raw videos (moving) by using the displacement vectors calculated with the motion tracking algorithm. Each video image of the original video sequence was

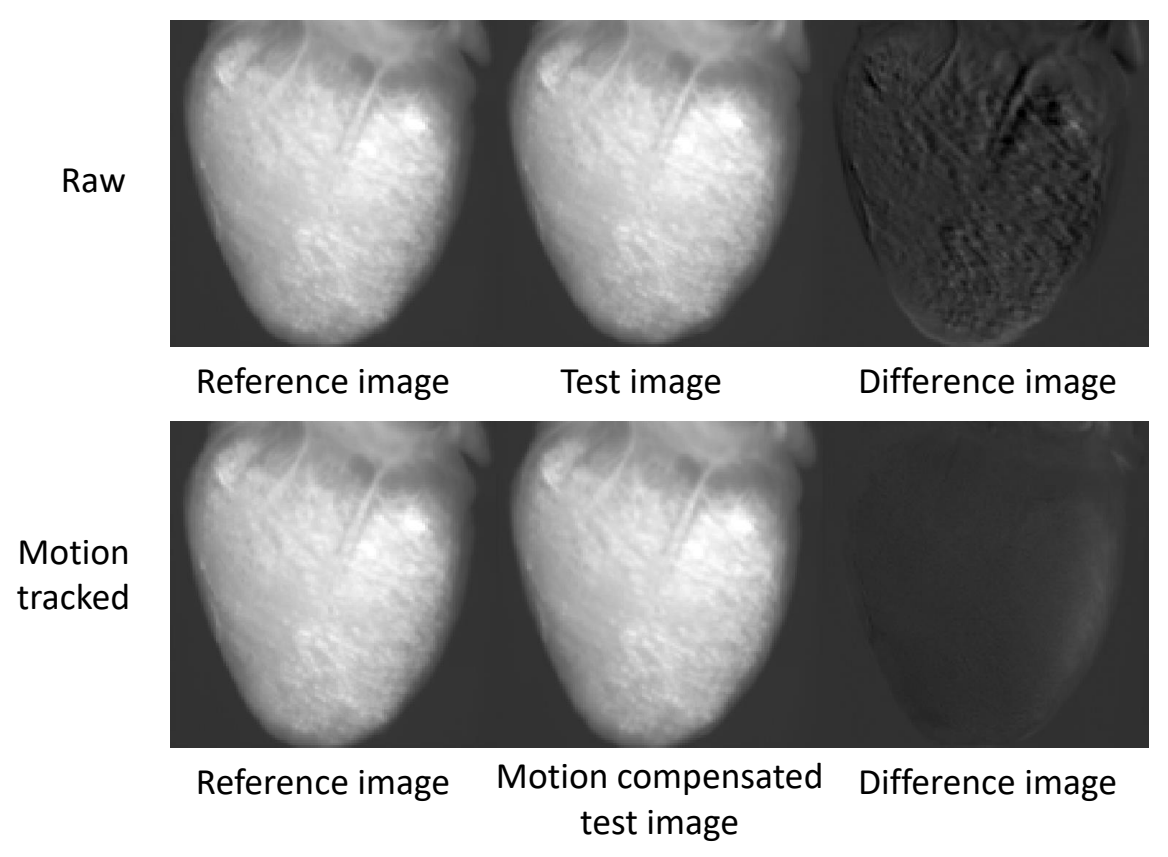

Fig. 4.2: Comparison of fluorescent images of a beating rabbit heart from an optical mapping experiment without motion tracking (upper row) and with motion tracking and compensation (lower row). The difference image on the upper row shows substantial pixel intensity differences between the test (at $300 \mathrm{~ms}$ ) and reference image (at $0 \mathrm{~ms}$ ) due to the relative motion of the contracting heart with respect to the camera. The difference image on the lower row shows comparatively negligible pixel intensity difference when the motion of the test image is tracked and compensated with respect to the reference image. The mean square error (mse) between test and reference image before tracking was 0.0015 and after motion tracking the error reduced to 0.0006 .

geometrically transformed using the tracked displacements to match the 
image in the reference frame. All registered video images were stacked to produce a final motion compensated video and it was stored separately for further analysis. An example of numerical motion tracking and motion compensation of a rabbit heart video image is shown in Figure 4.2. The upper row shows the difference between a test and reference image of a contracting heart before applying numerical motion tracking and motion compensation. The temporal difference between test and reference images is $300 \mathrm{~ms}$ (75 frames). The difference image (raw) is characterized by substantial pixel intensity variation due to the displacement of the test image upon contraction with respect to the reference image. On the other hand, motion compensated difference image on the lower row shows less variation in pixel intensity between the motion compensated test image and the reference image, indicating proper registration between the images with the application of motion tracking and motion compensation.

\subsection{Action potential wave propagation in motion tracked hearts}

Optical mapping experiments were conducted on contracting rabbit hearts with dual excitation wavelengths as shown in Figure 3.2 in order to do ratiometric action potential measurements. The heart was stained with voltage sensitive ratiometric dye Di-4-ANEPPS and the fluorescence signals corresponding to blue and green excitation light were collected with a single bandpass emission filter as shown in Figure 3.1. The optical mapping data captured with blue and green excitations were separated and stored as normalized videos with intensity values $I \in\left[\begin{array}{ll}0 & 1\end{array}\right]$ using equation (3.1). Motion tracking was applied on both blue and green elicited fluorescence video images separately with the first frame of each video sequence serving as the reference. Motion tracked blue and green action potential videos were compensated as mentioned in section 4.1.1.

An example of action potential wave propagation from a contracting heart before and after motion tracking for blue and green excitation wavelengths is shown in Figure 4.3. The action potential dependent fractional change in fluorescent signal $\Delta \mathrm{F}$ for blue excitation is less in magnitude (with positive sign), which makes it difficult to visualize the intensity variation in video 
A
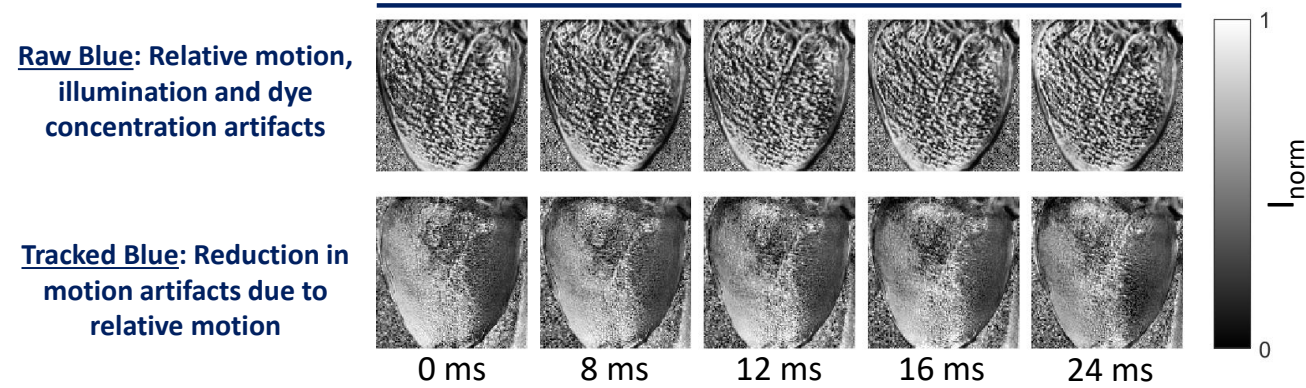

B

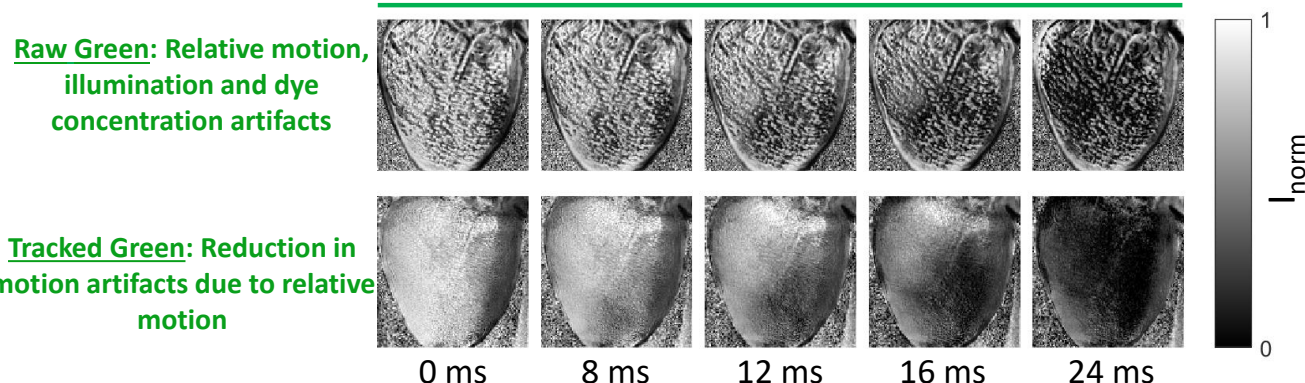

Fig. 4.3: Motion artifacts affecting action potential wave propagation. Comparison of wave propagation in contracting heart before (raw) and after (tracked) motion tracking for blue and green excitation wavelengths. A) Action potential wave propagation on a ventricular surface with blue excitation. Action potential before motion tracking (raw blue) shows significant motion artifacts $(\approx 2$ pixels representing $0.4 \mathrm{~mm}$ of cardiac tissue) as compared to motion tracked case (tracked blue). B) Action potential wave propagation with green excitation light. Dark areas represent depolarized tissue and bright areas correspond to repolarized tissue. All optical mapping images show normalized pixel intensities over time.

images. The $\Delta \mathrm{F}$ for green excitation is negative and hence the action potential wave propagation is characterized by decreasing intensity in the images from $t=0 \mathrm{~ms}$ to $t=24 \mathrm{~ms}$. The action potential wave propagation before motion tracking (raw blue and raw green) for blue and green excitation shows substantial distortions in action potential wave propagation due to the superposition of contractile motion with the action potential. The action potential wave propagation are affected by motion artifacts due to relative motion between the cardiac tissue and the detector, inhomogeneous dye concentration and illumination inhomogeneities. The action potential wave propagation with motion tracking (tracked blue and tracked green) show comparatively less motion artifacts due to the removal of relative motion artifacts present in the raw video images. However, dye concentration and illumination artifacts are still present in the video images as indicated by the 
non-uniform intensity variation across the heart in motion tracked images with an action potential depolarization (see Figure $4.3 \mathrm{~B}$ at $24 \mathrm{~ms}$ ).

\subsection{Marker-free motion tracking and excitation ratiometry}

As the motion tracking alone is not enough to produce artifacts-free action potentials, a combination of excitation ratiometry with marker-free motion tracking is employed to enhance action potential signal quality by removing the residual artifacts present in the signal. As mentioned in the previous section, motion tracked videos were obtained by tracking the motion of the cardiac tissue for blue and green excitation light separately. The motion tracked videos corresponding to action potentials for blue and green excitation are used to produce ratiometric video images. The ratio between these action potentials is calculated by dividing the action potentials for blue excitation light (positive $\Delta \mathrm{F}$ ) with action potentials for green excitation (negative $\Delta \mathrm{F}$ ). This convention is used in all ratio measurements throughout the thesis in order to produce an upward action potential depolarization (see Figure 4.4 A, ratio).

Figure 4.4 shows the effect of combined motion tracking and ratiometric measurements on action potential time series obtained during electrical pacing at a cycle length $C L=350 \mathrm{~ms}$. Figure $4.4 \mathrm{~A}$ shows the variation of action potential time traces in contracting (raw) and motion tracked conditions. The video images were normalized with the maximum and minimum intensity values from the entire video sequence as given in equation (3.1) before tracking the motion. The colors blue, green and black represent action potential time traces with blue excitation, green excitation and ratio (blue/green), respectively. Figure $4.4 \mathrm{~B}$ shows all the time traces shown in Figure $4.4 \mathrm{~A}$ by normalizing them in the interval [0 1] with a sliding window (by using the maximum and minimum value of action potential inside the window). The size of the sliding window is taken as the cycle length to make sure that there exists at least one action potential in each window. Green excitation results in downward action potential depolarization due to the reduction in fluorescent intensity with action potential depolarization (negative $\Delta \mathrm{F}$ ) as shown in Figure 3.1. Conversely, blue excitation produces upward action 
A
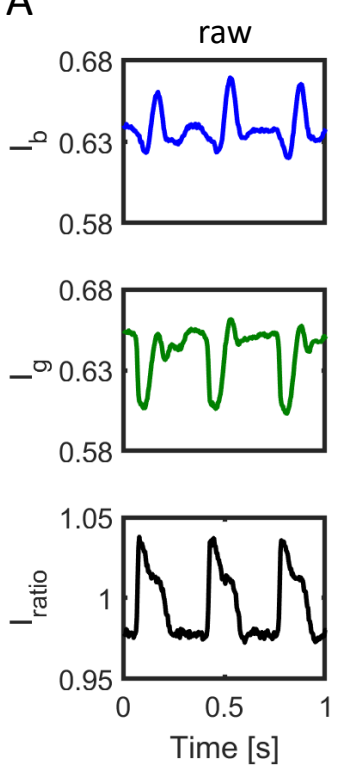
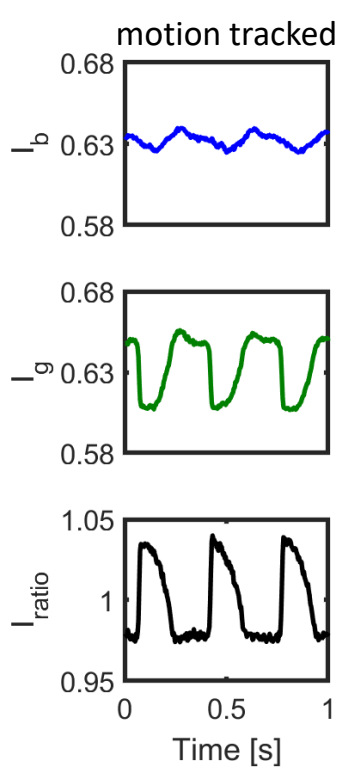

B
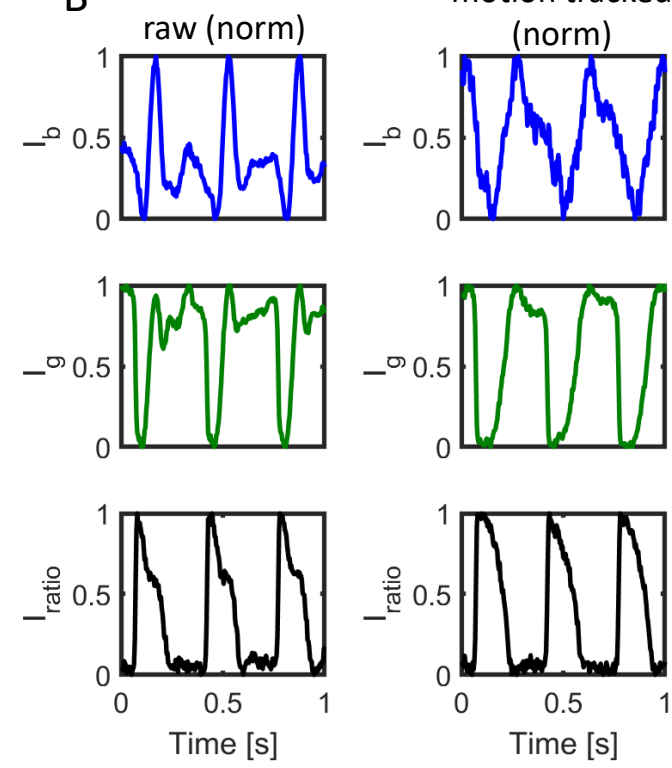

Fig. 4.4: Effects of motion tracking and ratiometry on optical action potential traces from a contracting heart at a pacing cycle length of $C L=350 \mathrm{~ms}$. Comparison of optical action potential time traces from a rabbit heart before and after motion tracking A) Action potential from a contracting heart before (raw) and after motion tracking (motion tracked). $I_{b}$ and $I_{g}$ represent the fluorescent intensity corresponding to blue and green excitation and $I_{\text {ratio }}$ is the ratio between optical action potentials obtained with blue and green excitation light. The optical action potential signal corresponds to blue excitation light produces a very small fractional change $(\Delta \mathrm{F} / \mathrm{F}<1 \%)$ of fluorescence with cardiac depolarization as compared to green light excitation $(\Delta \mathrm{F} / \mathrm{F} \approx 3 \%)$. Neither ratiometry nor motion tracking alone can remove all the motion artifacts but the combination of both these techniques reduces motion artifacts to a great extend. B) Action potential time traces obtained with sliding window normalization of signals shown in panel A.

potential depolarization due to the increase in fluorescent intensity with action potential depolarization. However, there is only a small fraction of action potential signal $(\Delta \mathrm{F} / \mathrm{F}<1 \%)$ with blue excitation light. The motion tracked data corresponding to blue excitation shows mostly residual artifacts due to the dye concentration and illumination changes which exist even after motion tracking and compensation. On the other hand, green elicited action potential shows comparatively higher fluorescent change $(\Delta \mathrm{F} / \mathrm{F} \approx 3 \%)$, which amplifies the action potential in the ratio signal and produces higher fractional change in fluorescence $(\Delta \mathrm{F} / \mathrm{F} \approx 4 \%)$. The motion tracked action 
potential with green excitation light $\left(I_{g}\right.$, motion tracked) shows significant improvement in signal quality compared to the raw signal $\left(\mathrm{I}_{\mathrm{g}}\right.$, raw) as visible by the change in shape of the action potential during repolarization. However, the baseline of the action potential at the diastolic interval is slightly distorted due to motion artifacts originating from nonuniform dye concentration and illumination changes. Motion artifacts arising from nonuniform dye concentration across the heart tissue are common in action potentials corresponding to blue and green excitation light.
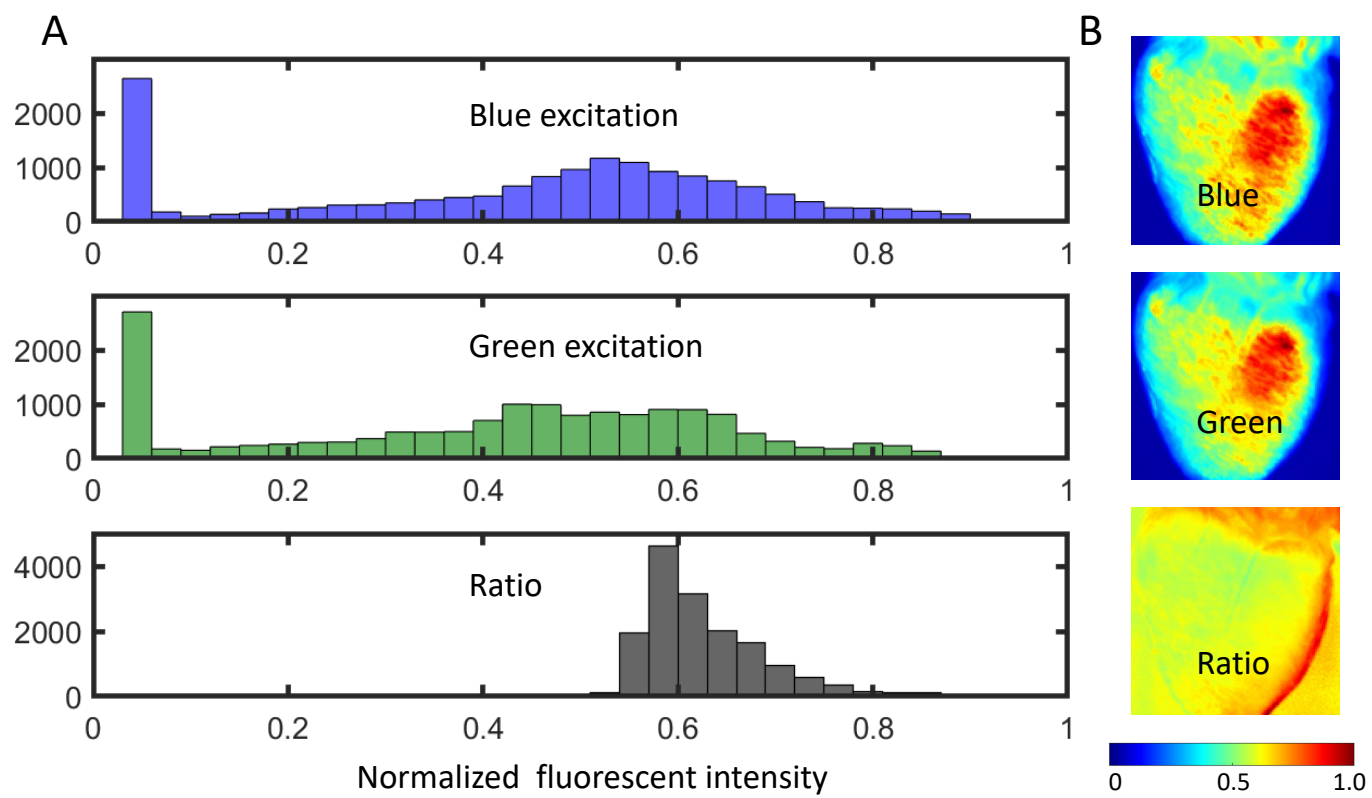

Fig. 4.5: Variation of fluorescent intensity across the heart tissue with blue excitation, green excitation and ratio. A) Normalized fluorescent intensity (pixel intensities were divided by the maximum intensity) calculated from an exemplary video images (snapshots) corresponding to blue excitation (upper row), green excitation (middle row) and ratio (lower row) as a histogram. B) The snapshots of the heart used to compute the histogram. The images from top to bottom represent normalized fluorescent intensity across the heart tissue for blue excitation, green excitation and ratio. The ratio image shows homogeneous intensity across the heart tissue.

However, motion artifacts originating from inhomogeneous illumination of the tissue are not common in these action potentials since the light intensity variations across the heart tissue with blue and green excitation light are not same. Hence, special care was taken during the experiment to arrange these light sources such that the ratio image shows nearly homogeneous intensity across the tissue. This was done by adjusting the blue and green light sources 
by looking at the ratio image. Figure 4.5 illustrates fluorescent intensity variations across heart tissue with blue excitation, green excitation and ratio. The ratio image shows homogeneous intensity distribution across the cardiac tissue as compared to fluorescent video images of blue and green excitation. Now, the illumination related artifacts and dye concentration artifacts are common in action potential signals produced with blue and green excitation light. Hence, taking the ratio between the action potentials corresponding to

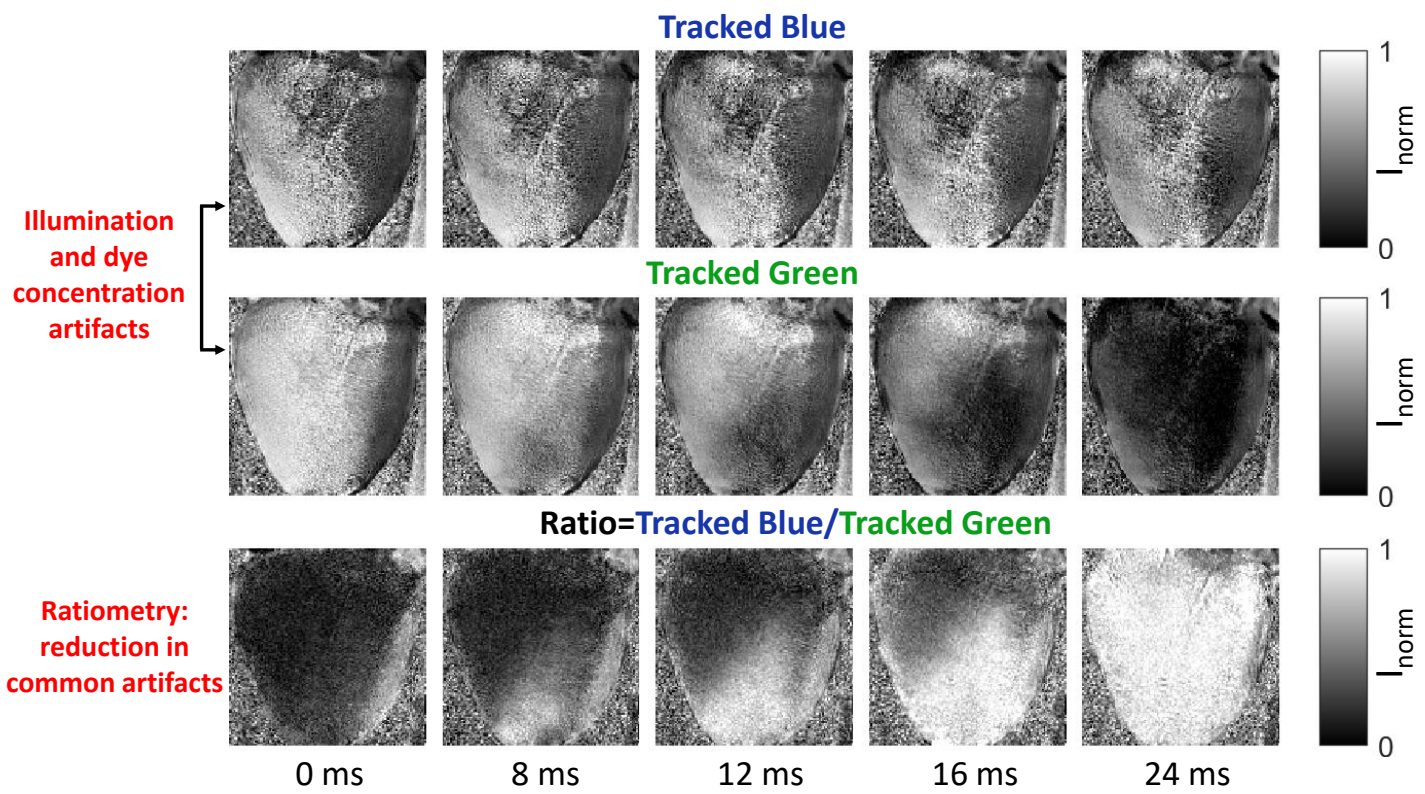

Fig. 4.6: Effect of combined motion tracking and excitation ratiometry on action potential wave propagation in contracting rabbit hearts. Motion tracked action potentials corresponding to blue (tracked blue) and green (tracked green) action potentials showing illumination and dye concentration artifacts. Ratiometric measurements illustrating reduced motion artifacts compared to motion tracked action potentials of blue and green excitation light. All optical mapping images show normalized pixel intensities over time. Bright areas corresponding to depolarized tissue in ratio whereas, dark areas corresponding to depolarized tissue in tracked green.

blue and green excitation in motion tracked condition ( $\mathrm{I}_{\text {ratio }}$, motion tracked) results in action potential time traces with significant reduction in motion artifacts. It is interesting to note that even without motion tracking, the ratio between raw action potentials produce time traces, which at least have similar morphology of motion tracked action potential. Nevertheless, motion tracking or ratiometry alone is not sufficient to remove all the motion related artifacts. 
The effect of combined motion tracking and ratiometry on action potential wave propagation (produced during electrical stimulation, $C L=350 \mathrm{~ms}$ ) is shown in Figure 4.6. Motion tracked action potentials with blue (tracked blue) and green (tracked green) excitation light contain illumination and dye concentration artifacts. Ratiometry removes these artifacts significantly (but illumination artifacts are only partially removed). The wave propagation across the heart becomes more homogeneous and consists of less motion artifacts compared to motion tracked action potentials of blue and green excitation light. As discussed in section 4.3, blue excitation produces smaller $\Delta \mathrm{F} / \mathrm{F}$ value.

\subsection{Comparison of action potential time traces between contracting and Blebbistatin- uncoupled hearts}

The action potential time traces obtained with combined motion tracking and excitation ratiometry are compared with action potentials obtained from Blebbistatin-uncoupled hearts, where the mechanical contraction is uncoupled pharmacologically from electrical activity. Figure 4.7 shows the comparison of action potentials from a contracting heart with motion tracking (motion tracked) and with Blebbistatin administration (Blebbistatin).

Colors blue, green and black represent action potentials with blue excitation, green excitation and ratio (blue/green), respectively. Figure $4.7 \mathrm{~A}$ shows this comparison in which action potential with motion tracking and ratiometry ( $\mathrm{I}_{\text {ratio }}$, motion tracked) shows similarity in morphology with that of Blebbistatin administrated heart ( $\mathrm{I}_{\text {ratio }}$, Blebbistatin). Figure $4.7 \mathrm{~B}$ shows the same time traces of Figure 4.7 A with sliding window normalization. The motion compensated action potentials (action potentials obtained with motion tracking and ratiometry) show significant reduction in motion related artifacts, which encourage the use of motion tracking and ratiometric techniques to measure electrophysiological parameters in a precise manner. And these techniques allow the simultaneous measurement of action potential and contractility of the hearts without using any pharmacological suppression. 
A
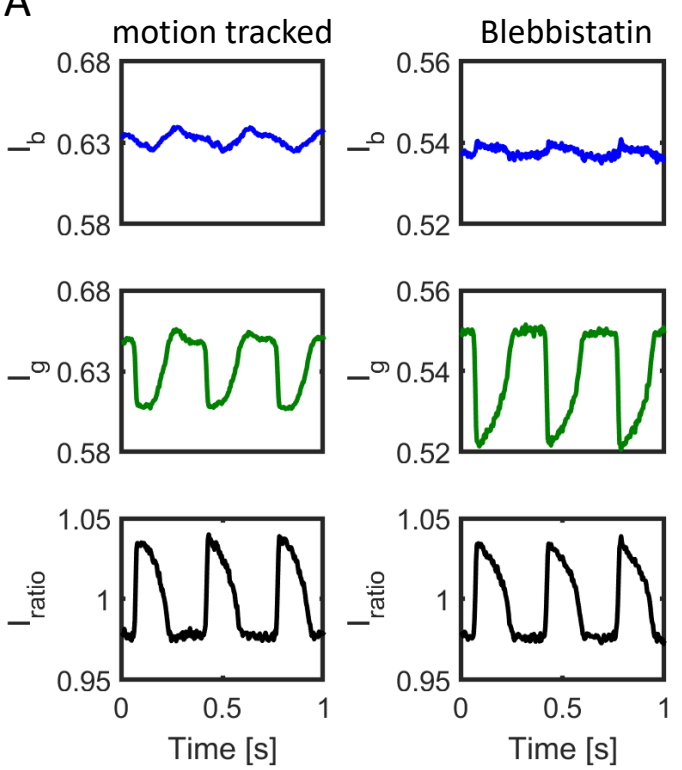
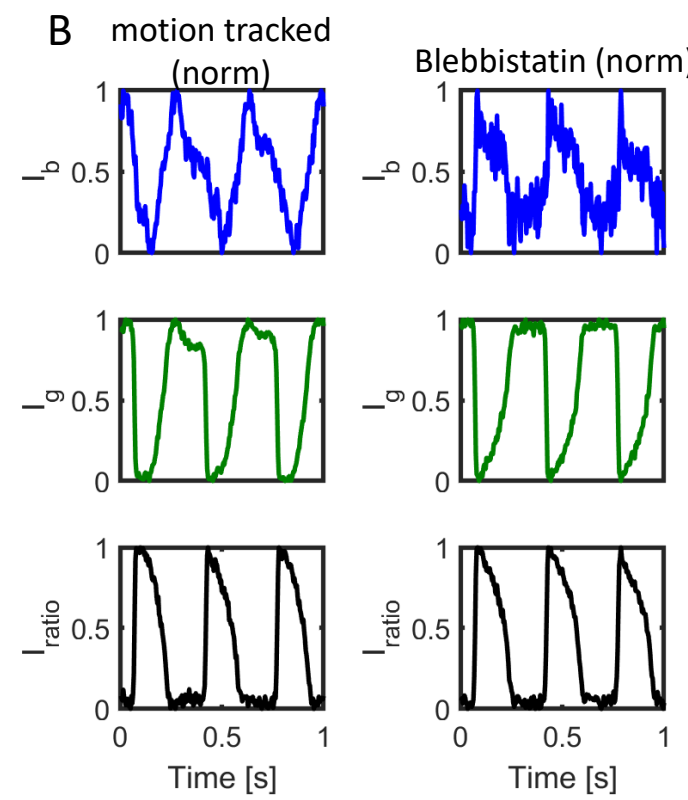

Fig. 4.7: Comparison of optical action potentials in motion tracked and Blebbistatinuncoupled rabbit hearts at a pacing cycle length of $C L=350 \mathrm{~ms}$. A) Action potential time traces with blue excitation (blue), green excitation (green) and ratio (black). The time traces obtained from the left ventricular surface by spatially averaging $3 \times 3$ pixels. The blue and green video images were normalized before motion tracking by using the maximum and minimum intensity value found in the entire video sequence. B) Time traces of panel A with sliding window normalization. Combined motion tracking and ratiometry produces action potential time series with negligible residual artifacts, which has similar morphology of that of action potential with Blebbistatin. 



\section{Comparison of APD restitution in}

contracting and Blebbistatin-uncoupled hearts

Restitution properties of the heart in a contracting condition are not well studied and high resolution optical mapping studies of cardiac restitution involving contraction without using markers are still missing due to the motion artifacts created by the mechanical contraction of the heart. This chapter mainly focuses on the precise measurement of action potential duration (APD) and cardiac restitution properties of a contracting heart by using combined marker-free motion tracking and ratiometric imaging techniques. Also, a systematic comparison of APDs and restitution properties of the heart in contracting and non-contracting (contraction inhibited by Blebbistatin) conditions is described in detail.

\subsection{APD and electrical restitution}

Electrical restitution is the dependence of heart's electrophysiological properties such as action potential duration (APD) or conduction velocity (CV) on pacing cycle length (CL). Though restitution properties of both APD and CV contain important information of the dynamics of the heart, this thesis is only focusing on APD restitution, which is the change in APD with CL (or change in APD with diastolic interval (DI)). APD is the duration of action potential (AP), usually represented with a number as a subscript indicating the percentage of repolarization of the cardiac tissue. For example, $\mathrm{APD}_{50}$ 
is the duration of $\mathrm{AP}$ at $50 \%$ repolarization, $\mathrm{APD}_{70}$ is the duration of $\mathrm{AP}$ corresponding to $70 \%$ repolarization and so on. CL is the time period of electrical stimulus used to pace (stimulate) the heart. Lowering the values of CL increases the pacing frequency $\left(f_{p}\right)$ since they follow a reciprocal relation. Diastolic interval (DI) is the relaxation time of the heart during diastole. The relationship between APD, DI and CL is shown in Figure 5.1.

While pacing the heart with electrical pulses of appropriate frequency (or pacing cycle length), the heart tries to capture the frequency and as a result if capture occurs, the heart beats at that particular frequency. This causes APD and DI to vary with CL by satisfying the following equation

$$
A P D+D I=C L
$$
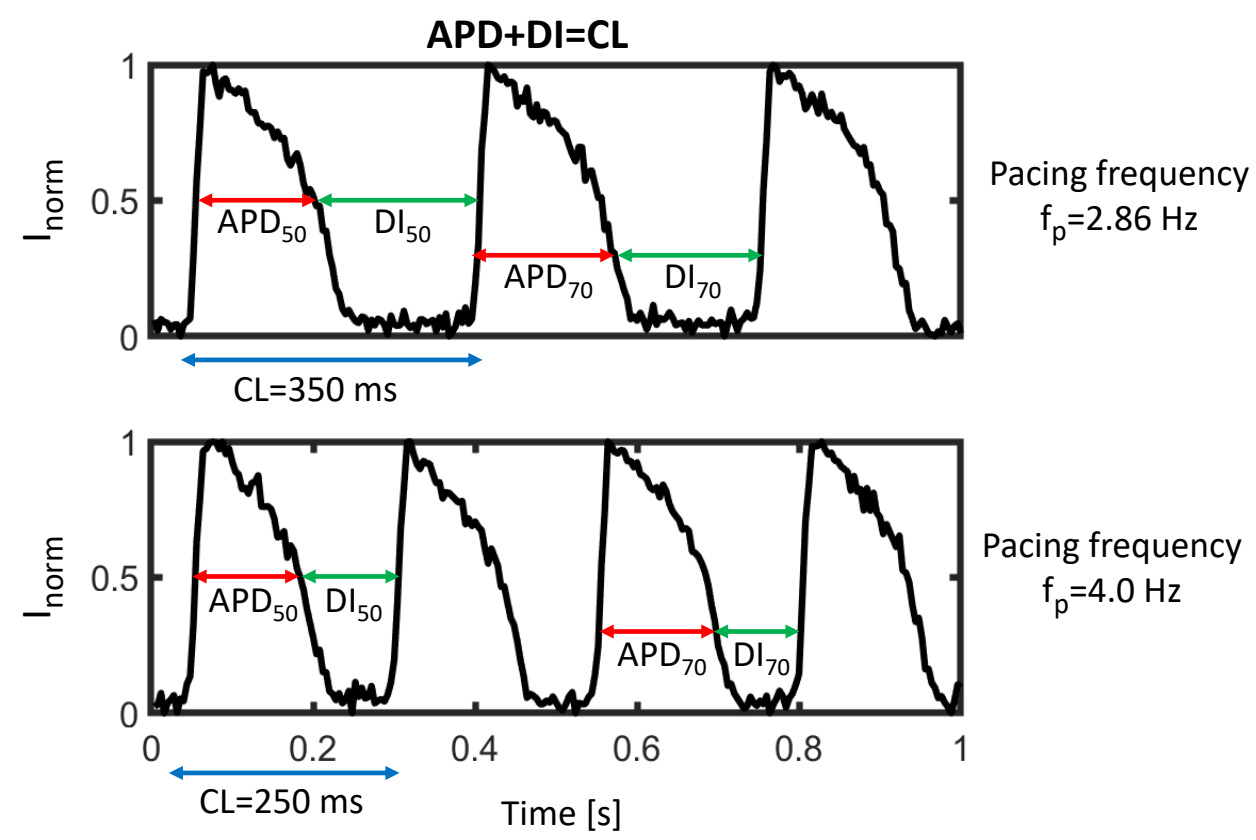

Fig. 5.1: Variation of action potential duration (APD) and diastolic interval (DI) with pacing cycle length (CL). Representative time series for two pacing cycle lengths $(C L=350 \mathrm{~ms}$ and $C L=250 \mathrm{~ms})$ showing APD and DI at $50 \%\left(\mathrm{APD}_{50}\right.$ and $\left.\mathrm{DI}_{50}\right)$ and $70 \%\left(\mathrm{APD}_{70}\right.$ and $\left.\mathrm{DI}_{70}\right)$ repolarization. Time series obtained from the left ventricular surface of a rabbit heart after the administration of Blebbistatin.

Figure 5.1 shows that both APD and DI shorten as we decrease the pacing cycle length from $C L=350 \mathrm{~ms}$ to $C L=250 \mathrm{~ms}$ (or increase the pacing frequency from $f_{p}=2.86 \mathrm{~Hz}$ to $f_{p}=4 \mathrm{~Hz}$ ). As we increase the frequency further, if the 
heart captures, the APD and DI go on decreasing until ventricular arrhythmia is reached. It can also happen that cardiac tissue is unable to capture the pacing when the frequency is very high (for rabbit hearts $f_{p}>6 \mathrm{~Hz}$ ). Hence to make sure that the heart captures for all the pacing frequencies to be investigated, the amplitude of the pacing pulses were kept at the maximum value in all the experiments.

There exist two standard protocols to measure the electrical restitution properties of the hearts, namely standard restitution and dynamic restitution [Kol+98; Osa12]. In standard restitution (S1-S2 restitution) protocol, the heart is paced with a train (10 or 20) of regular pulses (S1) at a constant pacing cycle length (usually, the shortest cycle length at which there are no beat to beat variations in APDs). Once steady state in APD and DI is reached, an extrastimulus (S2) is delivered to the tissue and the response of APD for S2 stimulus is recorded. This protocol is repeated with a progressively reduced S1-S2 coupling interval and the pacing is stopped once effective refractory period with no ventricular capture is reached [Raw16]. The APD for each S2 values is calculated and the restitution curve is plotted as either APD over S1-S2 or APD over preceding DI.

In dynamic restitution protocol, the cardiac tissue is paced with regular S1 pulses (usually 30 or 50 ) of constant cycle length. The cycle length (the S1-S1 interval) is progressively shortened until the heart fails to capture the pacing. The APD for each cycle length is calculated and the restitution curve is plotted as either APD over CL or APD over preceding DI.

Effects of these restitution protocols were well studied in different animal species using standard electrode measurement of monophasic action potentials. Since the objective of this thesis is not to compare different restitution protocols, the remaining part of the thesis will only focus on dynamic restitution.

\subsubsection{Dynamic restitution in optical mapping}

In order to assess the dynamic restitution during an optical mapping experiment in our lab, the heart was paced with a train of 50 biphasic electrical pulses of constant cycle length using a local pacing electrode (FHC Inc., USA) placed usually on the right ventricle. The pacing was started with a cycle length of $C L=500 \mathrm{~ms}$ and the cycle length was progressively shortened 
until $C L=100 \mathrm{~ms}$. The cycle length was decremented in steps of $50 \mathrm{~ms}$ until $C L=350 \mathrm{~ms}$. After that, the cycle length was decreased in the interval of 20 $m s$ from $C L=320 \mathrm{~ms}$ to $C L=200 \mathrm{~ms}$. The cycle length was further reduced in $5 \mathrm{~ms}$ intervals until $C L=100 \mathrm{~ms}$. The pacing was interrupted if the heart failed to capture the stimulus or if ventricular fibrillation was induced. After the restitution protocol was finished, APDs were calculated using custom developed MATLAB programs.

\section{Measurement of action potential duration}

In order to measure APD, the optical mapping data was first spatio-temporally filtered as explained in section 3.3.1 with a window size of $3 \times 3 \times 3$. The filtering was necessary to improve the accuracy of the APD calculation algorithm. After that, the action potential time traces were extracted across the cardiac tissue. For each cycle length, times series of each pixel (pixel which was filtered, not exactly single pixel) contain 50 elicited action potentials as a result of 50 electrical pacing stimuli. Out of these 50 action potentials, the last 30 action potentials were used for analysis. In order to calculate $\mathrm{APD}_{50}$, the upstroke and downstroke of each action potential at $50 \%$ height ( $50 \%$ repolarization) was calculated. $\mathrm{APD}_{50}$ was estimated as the difference in time corresponding to action potential upstroke and downstroke at $50 \%$ height. Similarly, $\mathrm{APD}_{70}$ was calculated from the upstroke and down stroke of the action potential at 30\% height (70\% repolarization) (see Figure 5.1 for the definition of $\mathrm{APD}_{50}$ and $\left.\mathrm{APD}_{70}\right)$.

\section{Electrical restitution of action potential duration}

An example of a electrical restitution curve after administrating the heart with Blebbistatin is shown in Figure 5.2 A. The $\mathrm{APD}_{50}$ is calculated for cycle lengths between $C L=500 \mathrm{~ms}$ to $C L=135 \mathrm{~ms}$. For cycle lengths below $C L=135 \mathrm{~ms}$, ventricular fibrillation was induced during the pacing protocol. Hence these cycle lengths were not used in the restitution analysis since the calculation of APD is difficult during irregular ventricular fibrillation. APD for each cycle length is calculated as the mean value of APDs from multiple pixels $(\approx 5400)$ on the left ventricular surface. Error bars represent the standard deviation of APDs at these pixels, indicating the heterogeneity in $\mathrm{APD}_{50}$ on the left ventricular surface (also minor measurement errors are also included). For 
each pixel, APD was calculated from 30 consecutive action potentials. These 30 action potentials were separated into odd and even action potentials in order to detect APD alternans. Panel B (zoomed version of panel A, for lower cycle lengths) shows APD alternans between $C L=160 \mathrm{~ms}$ to $C L=135 \mathrm{~ms}$, which are considered as the potential trigger of cardiac arrhythmias.
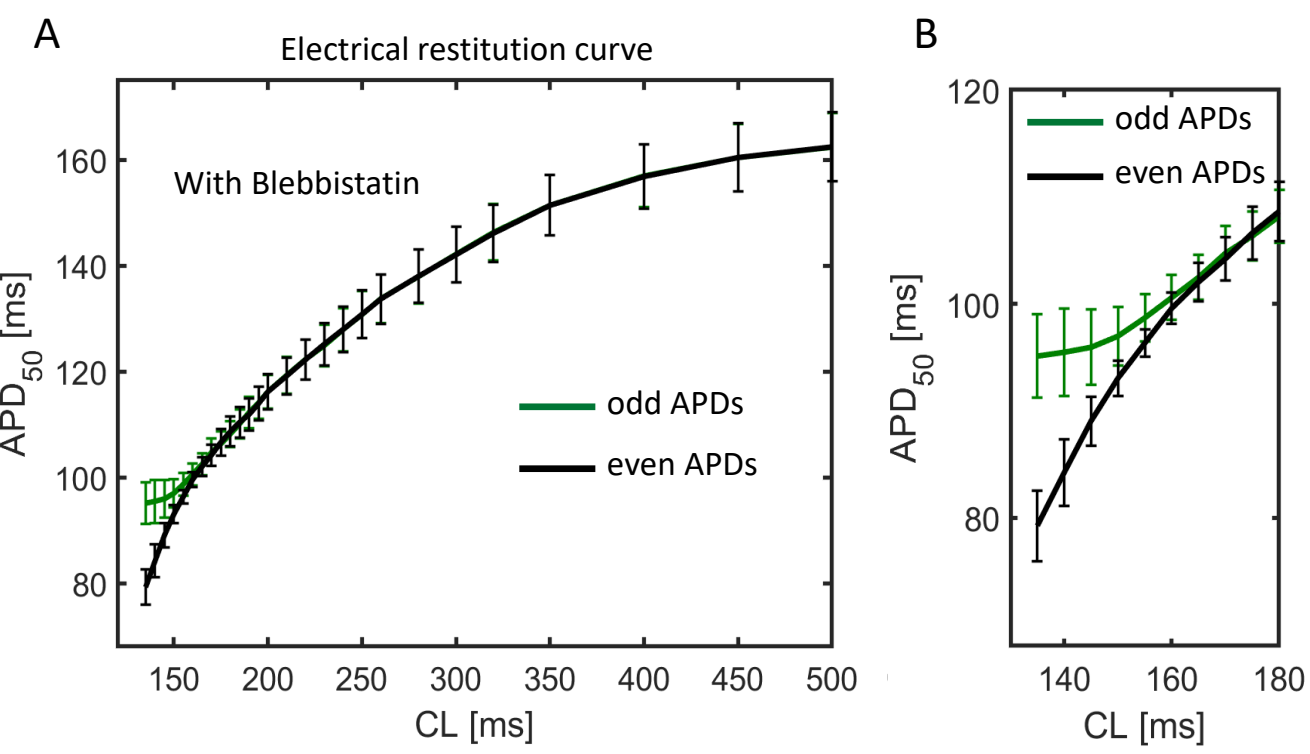

Fig. 5.2: APD restitution curve from a rabbit heart. The contraction of the heart was suppressed by the administration of Blebbistatin $(5.6 \mu \mathrm{M})$. A) APDs at $50 \%$ repolarization $\left(\mathrm{APD}_{50}\right)$ are plotted as a function of pacing cycle lengths within the interval $C L=500 \mathrm{~ms}$ to $C L=135 \mathrm{~ms}$. For each cycle length, the $\mathrm{APD}_{50}$ is calculated as the mean value of APDs of $\approx 5400$ pixels on the left ventricular surface. Error bars represent the standard deviation of APDs from these pixels. For each pixel, APD is calculated as the mean value of 30 consecutive action potentials (15 odd and 15 even). B) Zoomed version of panel A, for lower cycle lengths, indicating the presence of alternans in $\mathrm{APD}_{50}$.

\subsection{Comparison of APDs in contracting and Blebbistatin-uncoupled hearts during electrical pacing}

The robustness of combined motion stabilization and ratiometric techniques allows the precise measurement of electrophysiological quantities such as 
action potential duration (APD) from contracting and deforming hearts. Hence, this methodology is used to compare the action potential signals

A

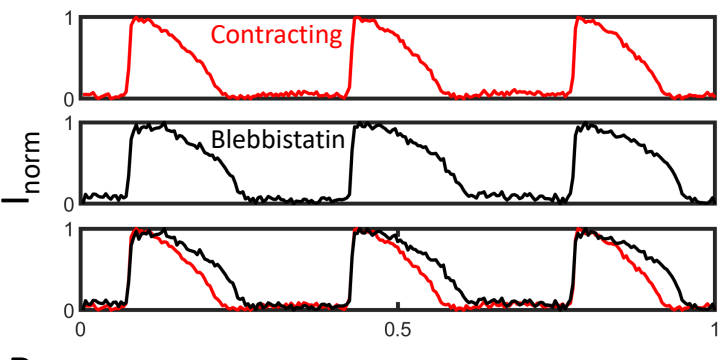

B

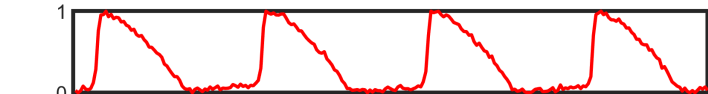

हํํำ

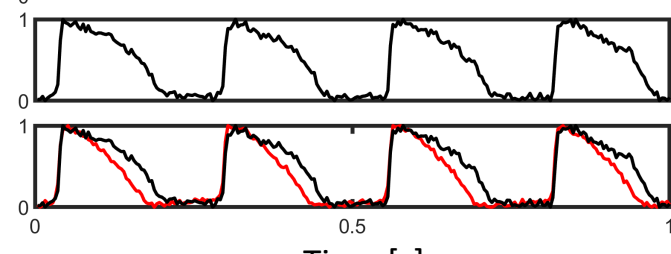

C

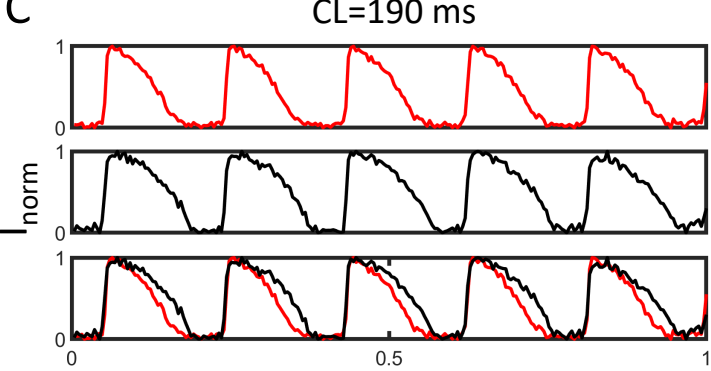

D

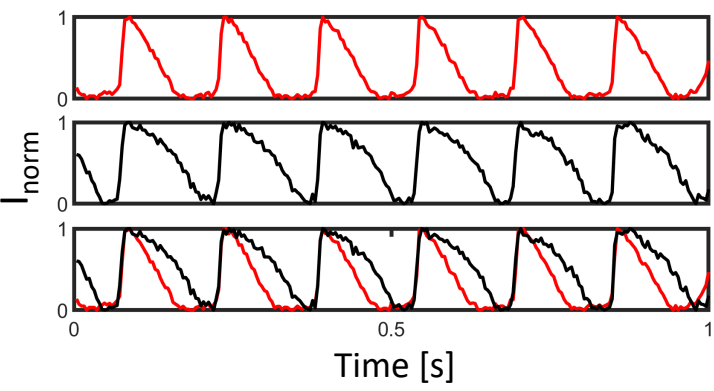

Fig. 5.3: Comparison of action potentials obtained through ratiometric optical mapping from the surface of a Langendorff perfused rabbit heart in contracting Blebbistatin-uncoupled conditions. The time series are obtained from the ratio between the action potentials corresponding to blue and green excitation wavelengths and are spatially averaged from $3 \times 3$ pixels A) Representative action potential time series during electrical pacing at a cycle length of $C L=350 \mathrm{~ms}$ (pacing frequency $f_{p}=2.86 \mathrm{~Hz}$ ). B) Time series of action potential at $C L=260 \mathrm{~ms}$ (pacing frequency $f_{p}=3.85 \mathrm{~Hz}$ ). C) Obtained action potential time series at a cycle length $C L=190 \mathrm{~ms}$ (pacing frequency $f_{p}=5.26 \mathrm{~Hz}$ ). D) Action potential time series at a cycle length $C L=155 \mathrm{~ms}$ (pacing frequency $f_{p}=6.45 \mathrm{~Hz}$ ). For all the cycle lengths, the APDs of the heart in Blebbistatin condition show significant prolongation compared to the APDs of the contracting heart. Also at higher frequencies, action potential time traces of the contracting heart become triangular and the difference between APDs of Blebbistatin and contracting heart increases.

in contracting and post Blebbistatin conditions. Figure 5.3 shows the comparison of action potential time traces obtained from the left ventricular surface of a rabbit heart in contracting (after applying motion stabilization and ratiometry) and non-contracting (electromechanically uncoupled with Blebbistatin) conditions. Panel A, B, C and D of Figure 5.3 show the action potential time series comparison in which the heart is electrically paced with 
the help of a local pacing electrode, with cycle lengths $C L=350 \mathrm{~ms}, C L=260$ $\mathrm{ms}, C L=190 \mathrm{~ms}$ and $C L=155 \mathrm{~ms}$, respectively. Action potential time series with Blebbistatin shows significant prolongation in action potential duration (APD) for all four cycle lengths. However, action potential shape becomes triangular at $C L=155 \mathrm{~ms}$ (or pacing frequency $f_{p}=6.45 \mathrm{~Hz}$ ). This is already a high frequency stimulation as compared to the normal rhythm frequency $(\approx 2 \mathrm{~Hz})$.

\subsection{Comparison of restitution curves between contracting and Blebbistatin-uncoupled}

\section{hearts}

The dependency of action potential duration over a range of pacing frequencies was calculated by electrically pacing the heart with progressively shortened cycle lengths. The dynamic restitution protocol as described in section 5.1.1 was used to electrically stimulate the heart, first in contracting and then in Blebbistatin-uncoupled conditions. The restitution curve obtained from a contracting heart is called electomechanical restitution to reflect the presence of mechanical contraction in this restitution measurements. Once again, marker-free motion tracking and ratiometric measurements were employed on contracting hearts. The action potential durations for $50 \%\left(\mathrm{APD}_{50}\right)$ and $70 \%\left(\mathrm{APD}_{70}\right)$ repolarization were calculated as described in section 5.1.1 from contracting and Blebbistatin-uncoupled hearts. Figure 5.4 shows the comparison of restitution curves of a single heart $(N=1)$ in contracting and Blebbistatin-uncoupled conditions (only $\mathrm{APD}_{50}$ is shown). The restitution curve shows shortening of $\mathrm{APD}_{50}$ in contracting hearts for all cycle lengths in the range $C L=500 \mathrm{~ms}$ to $C L=135 \mathrm{~ms}$. In other words, there is a prolongation in $\mathrm{APD}_{50}$ with the administration of Blebbistatin. Due to the induction of ventricular fibrillation during pacing, the $\mathrm{APD}_{50}$ measurement was not possible for cycle lengths $C L<135 \mathrm{~ms}$ in Blebbistatin-uncoupled condition. The restitution curve in contracting condition shows larger error bars in APD measurement in the interval $C L=320 \mathrm{~ms}$ to $C L=260 \mathrm{~ms}$ owing to the higher motion amplitude of the heart at these pacing cycle lengths (see Figure 5.5). 


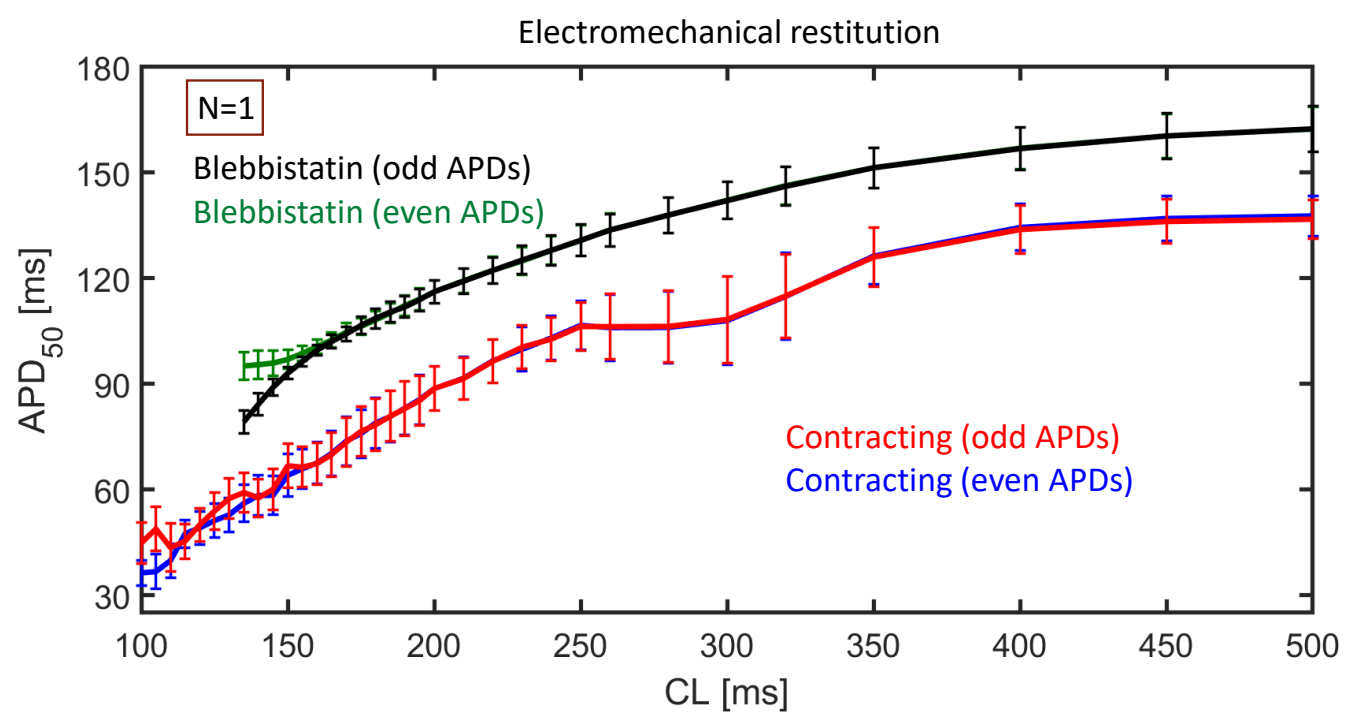

Fig. 5.4: APD restitution curve for a single rabbit heart in contracting (red and blue) and Blebbistatin-uncoupled (5.6 $\mu \mathrm{M}$; black and green) conditions. $\mathrm{APDs}$ at $50 \%$ repolarization $\left(\mathrm{APD}_{50}\right)$ are plotted as a function of pacing cycle lengths within the interval $C L=500 \mathrm{~ms}$ to $C L=100 \mathrm{~ms}$. Calculation of $\left(\mathrm{APD}_{50}\right)$ was not possible for $C L<135 \mathrm{~ms}$ in Blebbistatin administrated condition due to the induction of ventricular fibrillation during pacing at these cycle lengths. For each cycle length, the $\mathrm{APD}_{50}$ is calculated as the mean value of APDs of $\approx 5400$ pixels on the left ventricular surface. Error bars represent the standard deviation of APDs from these pixels. For each pixel, APD is calculated as the mean value of 30 consecutive action potentials (15 odd and 15 even). The APDs are divided into odd and even in order to detect APD alternans, which is visible at cycle lengths $C L<160$ $m s$ in post Blebbistatin condition, whereas in contracting (pre Blebbistatin) condition, no clear APD alternans is visible until $C L=110 \mathrm{~ms}$.

Alternans in $\mathrm{APD}_{50}$ can be seen in restitution curves corresponding to Blebbistatin conditions for cycle lengths in the pacing interval $C L=160 \mathrm{~ms}$ to $C L=135 \mathrm{~ms}$ as indicated by the deviation of odd (black) and even (green) APDs at these cycle lengths. Whereas, in contracting condition, no clear APD alternas is noticeable though the restitution curves slightly splits after $C L=110 \mathrm{~ms}$. Additionally, there was no induction of ventricular fibrillation during the restitution protocol with contracting heart. Hence, these restitution measurements show a link of APD alternans and cardiac arrhythmia susceptibility as shown in previous studies [Kar93; Kar94; Tse+16]. 
Though the heart used in Figure 5.4 is a typical example in terms of APD restitution, the APD alternans were not observed in all the hearts which were used to investigate restitution dynamics (observed in 2 out of 5 hearts). However, this example was used to illustrate the possibility of the generation of APD alternans and arrhythmias at high frequency electrical pacing.

\subsection{Variation of action potential duration and motion amplitude during electrical pacing}

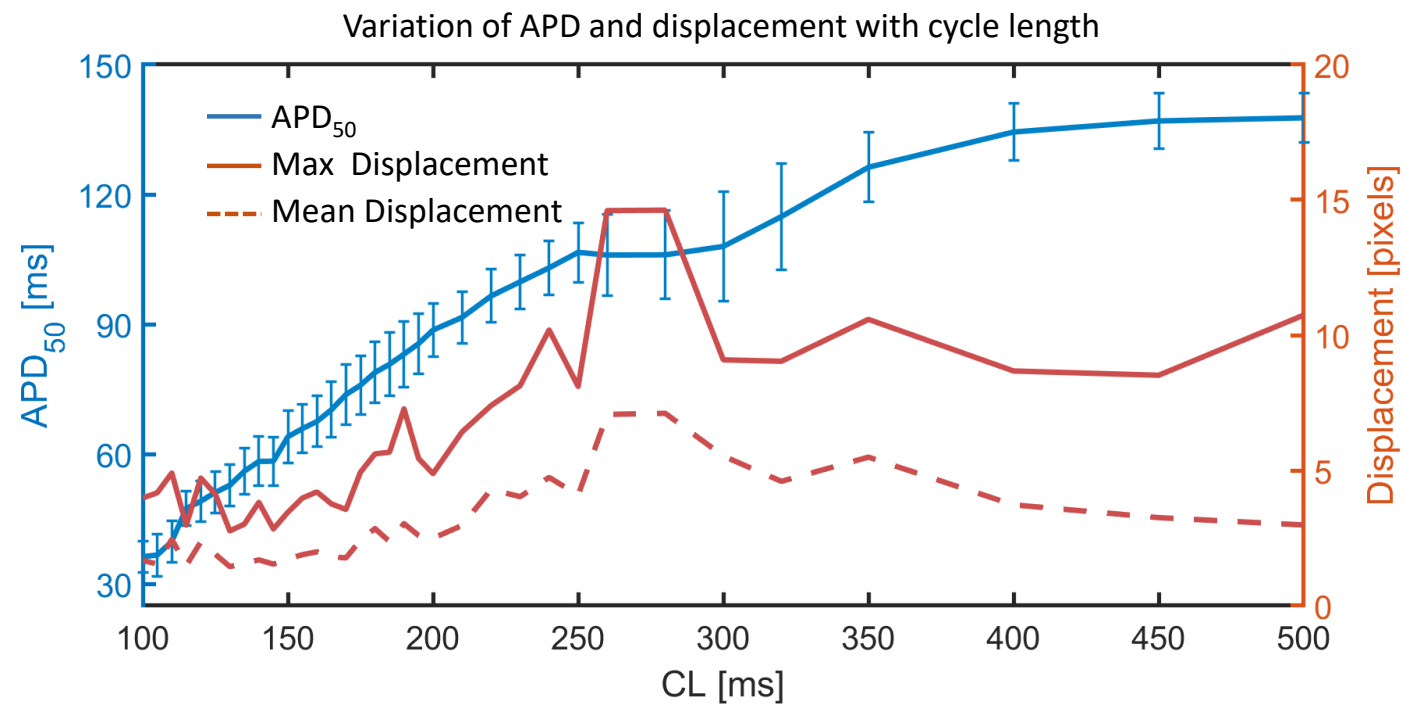

Fig. 5.5: APD restitution curve (blue) for a single rabbit heart (same as in Figure 5.4) in contracting condition and the displacement of the heart with pacing cycle length (reddish brown). APD for each cycle length is calculated from 15 consecutive odd action potentials. Error bars represent the standard deviation of APDs along the ventricular surface. The cycle lengths with larger error bars in the restitution curve coincide with displacement maxima indicating larger displacement and deformation of the heart resulting in less precise APD measurements.

Motion tracking produced displacements (in $x$ and $y$ directions) of the cardiac tissue for each pixel and for each frame with respect to the reference frame. The magnitudes of displacements in $x$ and $y$ directions were used to calculate the resultant displacement vector $\vec{d}(x, y, t)$. The magnitude of 
the resultant displacement vector (in pixels) representing the amplitude of motion (motion amplitude) is calculated for all the pacing cycle lengths in the range $C L=500 \mathrm{~ms}$ to $C L=100 \mathrm{~ms}$ (pacing frequency in the range $f_{p}=2 \mathrm{~Hz}$ to $10 \mathrm{~Hz}$ ). For each cycle length, the time varying motion amplitude (see Figure 5.11) is averaged across the cardiac tissue (mean value is calculated from all the pixels) and the effective motion amplitude is calculated either as the maximum value of displacement (Max Displacement, reddish brown, solid) or mean value of displacement (Mean Displacement, reddish brown, dashed), along the frames. Figure 5.5 shows the variation of motion amplitude (displacement in pixels) and action potential duration $\left(\mathrm{APD}_{50}\right.$, blue) with pacing frequencies for the same heart, which is used in Figure 5.4. The $\mathrm{APD}_{50}$ values are same as mentioned there (contracting condition), but only odd APDs are shown here. The $\mathrm{APD}_{50}$ and motion amplitude in general decrease with decrease in pacing cycle length (or with an increase in pacing frequency). But there exists a region of pacing interval ( $C L=320 \mathrm{~ms}$ to $C L=260 \mathrm{~ms}$ ) characterized by a sharp drop in $\mathrm{APD}_{50}$ values with higher errorbars. The larger errorbars of the restitution curve in this region coincide with a peak in motion amplitude for nearly the same cycle lengths, confirming that, higher amplitudes of contractile motion at these pacing frquencies cause inaccuracy in APD measurements. These larger motion amplitudes at these frequencies $(\approx 3 \mathrm{~Hz}$ to $4 \mathrm{~Hz})$ are due to the mechanical resonance phenomenon associated with the contracting heart, while hanging in a perfusion system.

\subsection{Comparison of APD maps in contracting and Blebbistatin-uncoupled hearts}

The action potential duration $\left(\mathrm{APD}_{50}\right)$ was calculated for all pixels in the camera field of view representing the left ventricular surface in contracting and Blebbistatin-uncoupled conditions. Figure 5.6 shows a typical example of APD maps obtained with four different pacing cycle lengths at these conditions. The analysis was performed for $N=5$ hearts and the observation was consistent in all the hearts.

APD maps show the variation of $\mathrm{APD}_{50}$ across the left ventricular surface of a rabbit heart (different from that shown in Figure 5.5) represented by $\approx 2900$ pixels $\left(\approx 1.3 \mathrm{~cm}^{2}\right)$. APD for each pixel was calculated from the 

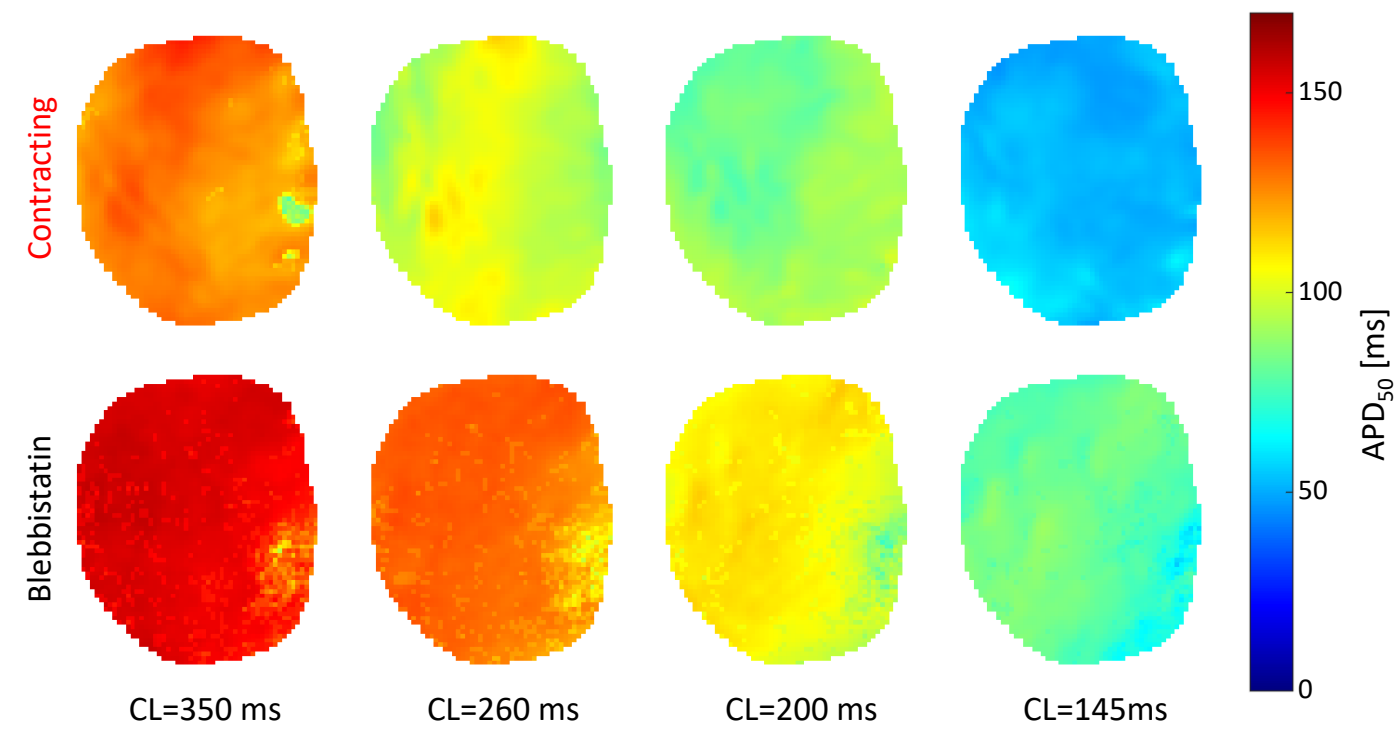

Fig. 5.6: Comparison of APD maps in contracting and Blebbistatin-uncoupled (5.6 $\mu \mathrm{M})$ conditions for different pacing cycle lengths. Blebbistatin prolongs action potential duration in all cycle lengths. Colorbar shows $\mathrm{APD}_{50}$ in $m s$. Each APD map shows APD values of $\approx 2900$ pixels, depicting the portion of left ventricular surface $\left(\approx 1.3 \mathrm{~cm}^{2}\right)$.

action potential time series of that particular pixel as described in section 5.1.1. The average values of $\mathrm{APD}_{50}$ (mean value calculated from APD maps) in contracting condition are $126 \pm 6.72 \mathrm{~ms}, 99 \pm 5.68 \mathrm{~ms}, 87 \pm 4.91 \mathrm{~ms}$ and $53 \pm 3.09 \mathrm{~ms}$ for cycle lengths $C L=350 \mathrm{~ms}, C L=260 \mathrm{~ms}, C L=200 \mathrm{~ms}$ and $C L=$ $145 \mathrm{~ms}$, respectively. Similarly, the average values of $\mathrm{APD}_{50}$ in Blebbistatinuncoupled condition are $152 \pm 6.76 \mathrm{~ms}, 130 \pm 6.16 \mathrm{~ms}, 106 \pm 6.0 \mathrm{~ms}$ and $80 \pm 5.29 \mathrm{~ms}$ for cycle lengths $C L=350 \mathrm{~ms}, C L=260 \mathrm{~ms}, C L=200 \mathrm{~ms}$ and $C L=145 \mathrm{~ms}$, respectively. The APD maps depict the prolongation of APDs in Blebbistatin-uncoupled condition as compared to contracting condition for all the pixels across the heart tissue and for all cycle lengths. On average, the APD maps show $22.75 \%$ shortening of $\mathrm{APD}_{50}$ in contracting condition as compared to Blebbistatin-uncoupled condition. Figure 5.7 shows the $\mathrm{APD}_{50}$ values of Figure 5.6 in contracting and Blebbistatin-uncoupled conditions as histograms. $\mathrm{APD}_{50}$ values in contracting condition are shorter than Blebbistatin-uncoupled condition for all cycle lengths used to pace the heart (but only four cycle lengths are shown here). Additionally, APDs in contracting condition, on average, show higher magnitudes of dispersion compared to Blebbistatin condition. 

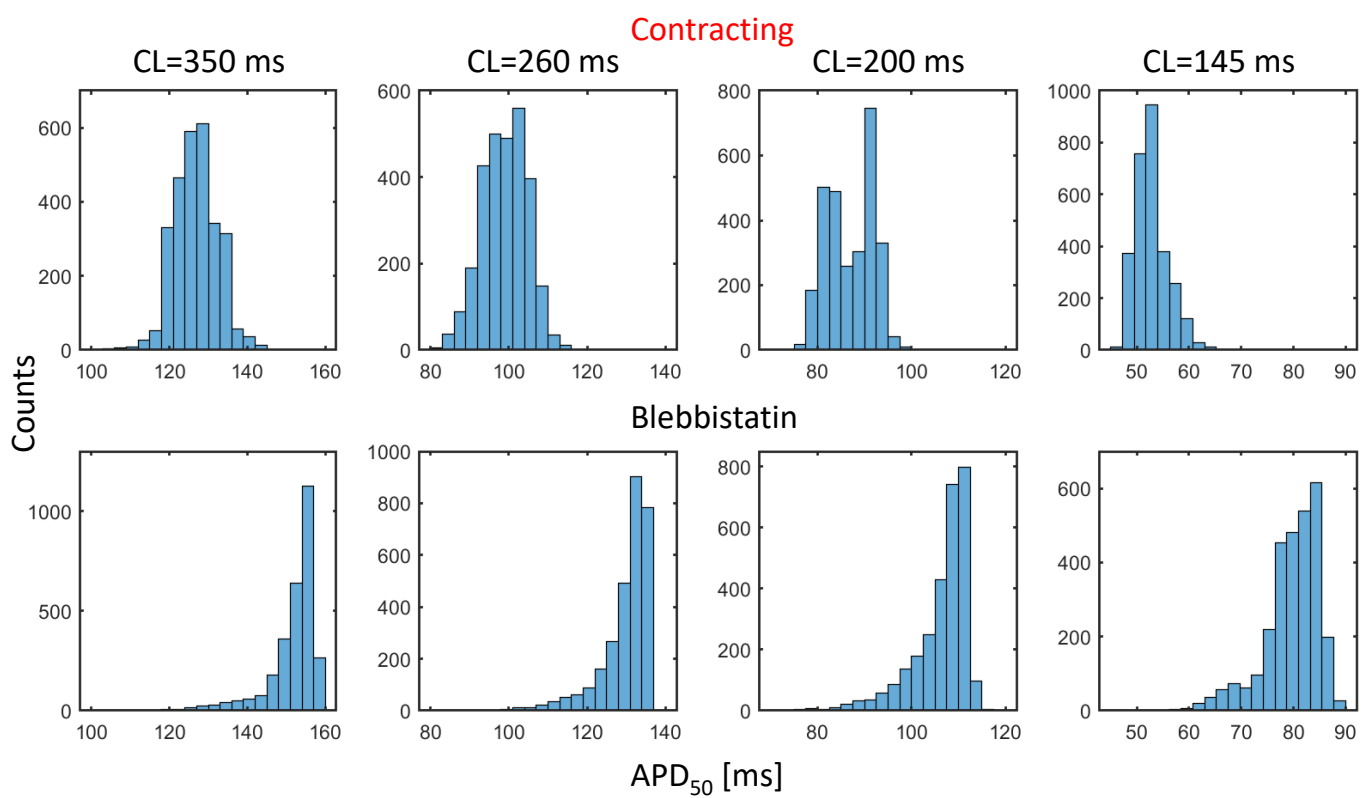

Fig. 5.7: Histogram of $\mathrm{APD}_{50}$ calculated from $\mathrm{APD}$ maps in contracting (top row) and Blebbistatin (bottom row) conditions for four pacing cycle lengths. $\mathrm{APD}_{50}$ values show prolongation in Blebbistatin condition for all cycle lengths.

\subsection{Electromechanical restitution curves from multiple hearts}

The restitution measurements in contracting and Blebbistatin-uncoupled conditions were repeated for multiple hearts $(N=5)$ and the comparison results are displayed in Figure 5.8. APD values are calculated from 30 consecutive action potentials out of 50 elicited action potentials by the application of 50 electrical stimuli as mentioned in section 5.1.1.

Panel A shows the average restitution measurements of $\mathrm{APD}_{50}$ from $N=5$ hearts in contracting (red) and Blebbistatin-uncoupled conditions (black). The APD values are pooled from all the pixels (pixels used to make APD maps) and all the hearts. Each data point is the mean of pooled $\mathrm{APD}_{50}$ corresponding to each cycle length and the error bars are standard deviations from these pooled APD values. Higher magnitudes of error bars are due to variations of APD values between different hearts. Even with the higher error bars, there exists a significant prolongation of APD values with the administration of Blebbistatin (or a shortening in contracting hearts as compared to Blebbistatin-uncoupled condition). 

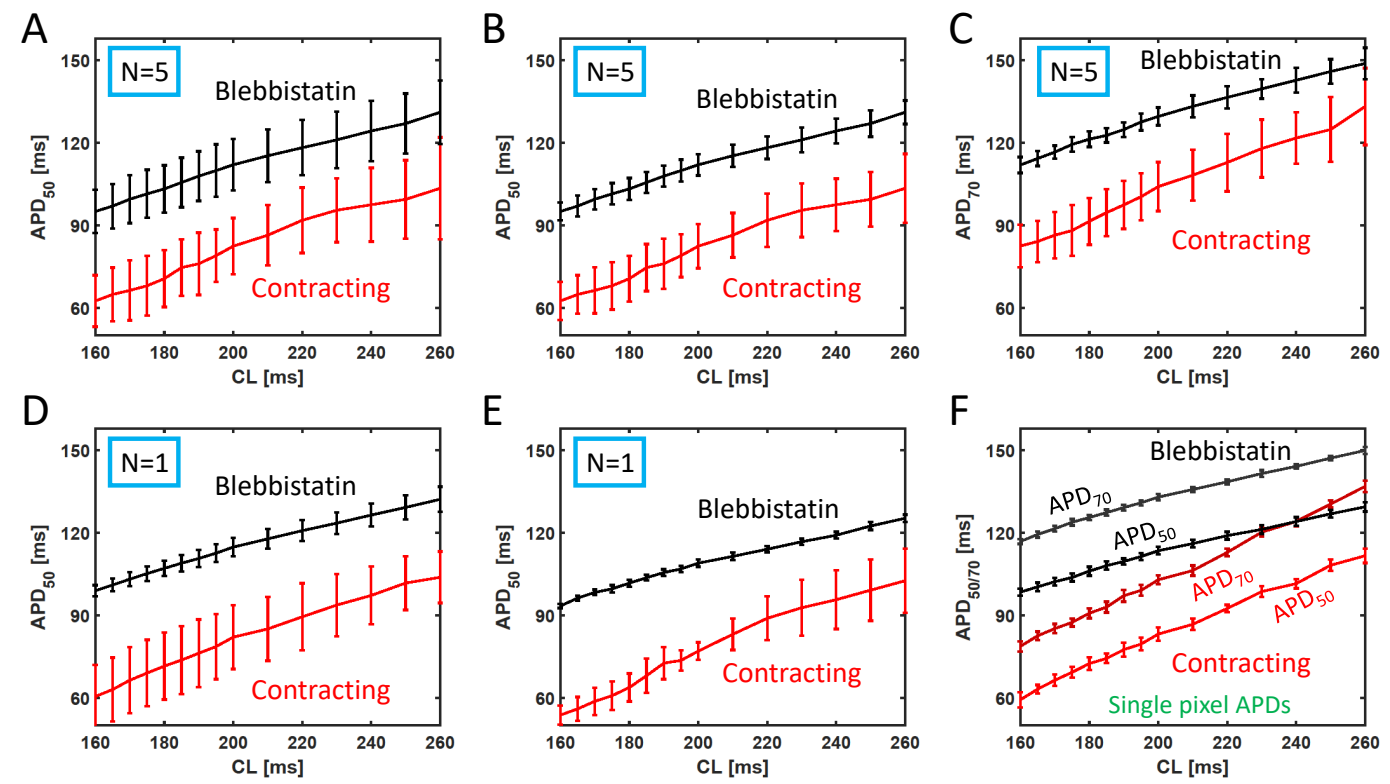

Fig. 5.8: Comparison of cardiac APD restitution curves for rabbit hearts in contracting (red) and Blebbistatin (black) treated conditions for progressively shortened pacing cycle lengths from $C L=260 \mathrm{~ms}$ to $C L=160 \mathrm{~ms}$ (pacing frequency $f_{p}=3.84 \mathrm{~Hz}$ to $f_{p}=6.25 \mathrm{~Hz}$ ). APDs calculated from 30 consecutive action potentials (obtained from optical mapping data) for each cycle length. A) Average $\mathrm{APD}_{50}$ restitution curve from $\mathrm{N}=5$ hearts. Error bars represent the standard deviation of all APDs pooled across all the pixels and all the hearts. Large standard deviations indicate the dispersion of APDs across multiple hearts. B) Average $\mathrm{APD}_{50}$ restitution curve for $\mathrm{N}=5$ hearts. Error bars represent the mean of standard deviations of $\mathrm{APD}_{50}$ from individual hearts. C) Average $\mathrm{APD}_{70}$ restitution curve for $\mathrm{N}=5$ hearts. Error bars represent the mean of standard deviations of $\mathrm{APD}_{70} \mathrm{~s}$ from individual hearts. D-E) Comparison of $\mathrm{APD}_{50}$ restitution curves for two different hearts. The error bars are calculated as the standard deviation of APDs along the pixels F) Comparison of single pixel $\mathrm{APD}_{50}$ and $\mathrm{APD}_{70}$ restitution curves.

Panel B represents the average restitution curves of $\mathrm{APD}_{50}$ from $N=5$ hearts (data points are same as in panel A) in contracting (red) and Blebbistatinuncoupled (black) condition, with errorbars calculated as the mean of standard deviations of APDs from these 5 hearts. The standard deviation for each heart is calculated from $\mathrm{APD}_{50}$ values of all the pixels. The comparatively larger error bars show the measurement uncertainty in contracting hearts due to residual motion artifacts. However, the restitution curves significantly differ between contracting and Blebbistatin conditions.

Panel C displays the average restitution curves of $\mathrm{APD}_{70}$ (contracting: red, 
Blebbistatin: black) for $N=5$ hearts, data points are averages of pooled $\mathrm{APD}_{70}$ from all the pixels (same pixels used for $\mathrm{APD}_{50}$ calculation) and all the hearts. Error bars represent mean of the standard deviations of all the $N=5$ hearts. The standard deviation for each heart is calculated from $\mathrm{APD}_{70}$ values of all the pixels.

Panel D and E represent the comparison of $\mathrm{APD}_{50}$ restitution curves in contracting and Blebbistatin-uncoupled conditions for two different hearts. Data points are the mean $\mathrm{APD}_{50}$ along the pixels and error bars represent the standard deviations of $\mathrm{APD}_{50}$ from all the pixels. Larger error bars in contracting condition show the measurement inaccuracy owing to the residual motion artifacts which persist in some pixels even after motion compensation.

Panel F shows the single pixel restitution curves of $\mathrm{APD}_{50}$ and $\mathrm{APD}_{70}$ in contracting and Blebbistatin-uncoupled conditions. APDs $\left(\mathrm{APD}_{50}\right.$ and $\left.\mathrm{APD}_{70}\right)$ show prolongation in Blebbistatin-uncoupled condition as compared to contracting condition. The mean errorbars computed with contracting hearts are $2.1 \pm 0.4$ and $1.7 \pm 0.2$ for $\mathrm{APD}_{50}$ and $\mathrm{APD}_{70}$, respectively. And the average error bars in Blebbistatin-uncoupled condition are $1.4 \pm 0.15$ and $1.0 \pm 0.13$ for $\mathrm{APD}_{50}$ and $\mathrm{APD}_{70}$, respectively. The smaller error bars (in contracting and Blebbistatin-uncoupled conditions) show the accuracy of single pixel APD calculation.

\subsection{Variation of action potential duration and restitution properties with Blebbistatin concentration}

To analyze the variation of action potential duration with the change in Blebbistatin concentrations, the Blebbistatin was administrated to the contracting hearts in multiple steps such that at each step $0.7 \mu \mathrm{M}$ of Blebbistatin was added to the perfusion bath until a final concentration of $2.8 \mu \mathrm{M}$ was reached, where the amplitude of contraction became small $(\approx 1$ pixel). There was a waiting time of about 20 minutes after adding each Blebbistatin concentration in order to give enough time for Blebbistatin's action. The hearts were progressively paced first in contracting condition and then with each Blebbistatin concentrations using the dynamic restitution protocol. The elec- 
tromechanical properties of the hearts were calculated after compensating the motion using motion tracking and ratiometry in contracting condition as well as in all the Blebbistatin concentrations. Figure 5.9 shows the variation of action potential duration, amplitude of contractile motion and restitution curve with the concentration of Blebbistatin (red: $0 \mu \mathrm{M}$ (contracting), light gray-black: Blebbistatin 0.7-2.8 $\mu \mathrm{M}$ )

Panel A shows the variation of a single action potential trace (obtained during electrical pacing with a cycle length $C L=180 \mathrm{~ms}$ in optical mapping experiments) with different Blebbistatin concentrations ( $0 \mu \mathrm{M}, 0.7 \mu \mathrm{M}, 1.4 \mu \mathrm{M}$, $2.1 \mu \mathrm{M}$ and $2.8 \mu \mathrm{M}) .0 \mu \mathrm{M}$ corresponds to fully-contracting condition of the heart in the absence of Blebbistatin. The time traces (optical action potential, $I_{\text {norm }}$ is the normalized fluorescent intensity modulated by action potential) were obtained after spatial averaging of $3 \times 3$ pixels from the left ventricular surface. The action potential duration $\left(\mathrm{APD}_{50}\right)$ in contracting condition is 68 $m s . \mathrm{APD}_{50}$ increases with each Blebbistatin concentration such that $\mathrm{APD}_{50}$ becomes $80 \mathrm{~ms}$ with $0.7 \mu \mathrm{M}, 96 \mathrm{~ms}$ with $1.4 \mu \mathrm{M}$ and $100 \mathrm{~ms}$ with both 2.1 $\mu \mathrm{M}$ and $2.8 \mu \mathrm{M}$, indicating a saturation in APD values for higher Blebbistatin concentration.

Panel B shows the variation of contractile motion amplitude of the heart with Blebbistatin concentration. The motion amplitude was calculated as mentioned in section 5.4. Upper row shows the maximum amplitude of motion, whereas lower row shows mean amplitude of motion for $C L=180$ $m s$. The maximum amplitude motion decreases from $\approx 5$ pixels to $\approx 1$ pixel and the average amplitude of motion decreases from $\approx 3$ pixels to $\approx 0.6$ pixel for the change in Blebbistatin concentration from $0 \mu \mathrm{M}$ to $2.8 \mu \mathrm{M}$.

Panel C represents the variation of $\mathrm{APD}_{50}$ restitution curve with Blebbistatin concentration. Restitution curves show prolongation of $\mathrm{APD}_{50}$ with Blebbistatin in a dose-dependent manner. Error bars become smaller with each concentration for all cycle lengths showing the reduction of residual motion artifacts as a consequence of reduction in motion amplitude as shown in panel B. 

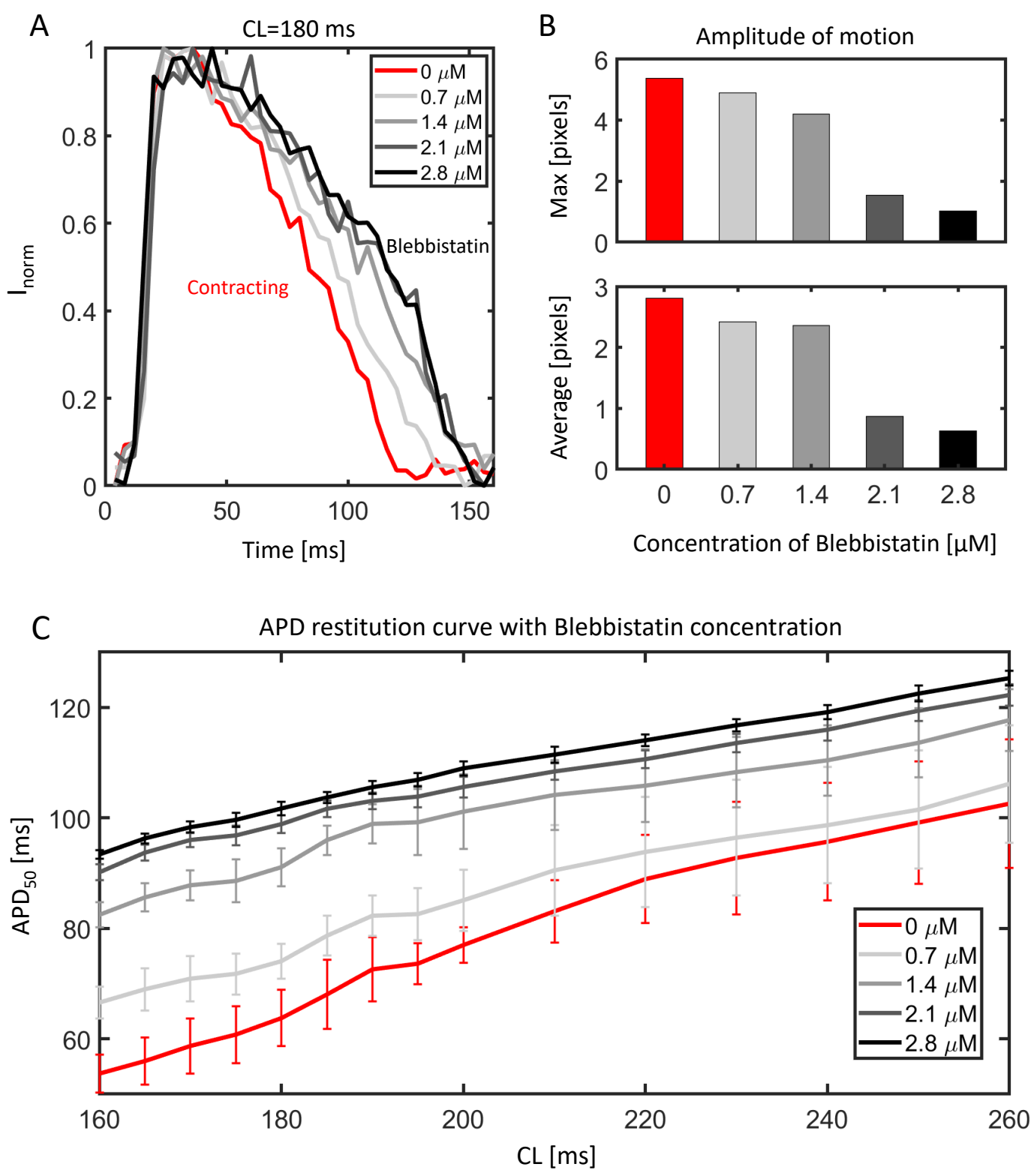

Fig. 5.9: Effect of Blebbistatin concentration $(0 \mu \mathrm{M}-2.8 \mu \mathrm{M})$ on the electromechanical properties of the cardiac tissue. A) Dependence of action potential duration $\left(\mathrm{APD}_{50}\right)$ on Blebbistatin concentration during electrical pacing with $C L=180 \mathrm{~ms}$. $I_{\text {norm }}$ represents the action potential modulated fluorescent intensity (normalized). Time traces were obtained after spatial averaging of $3 \times 3$ pixels from the left ventricular surface. B) Variation of the contractile motion amplitude (maximum and average) with the concentration of Blebbistatin. C) Variation of $\mathrm{APD}_{50}$ restitution curve with Blebbbistatin concentration showing the prolongation of $\mathrm{APD}_{50}$ for all cycle lengths. 


\subsection{Variation of action potential duration with Blebbistatin washout}

To examine the reversibility of electrophysiolgical properties of the hearts, Blebbistatin was washed out from $N=2$ hearts by perfusing the hearts with a fresh Tyrode solution for 60-100 minutes. The hearts started to beat during the washout process and the dynamic restitution protocol was used to electrically pace the hearts. Motion tracking and ratiometric measurements were applied on these moving hearts in order to measure the electrophysiological properties. The action potential signals produced during washout were compared with action potentials obtained with Blebbistatin.

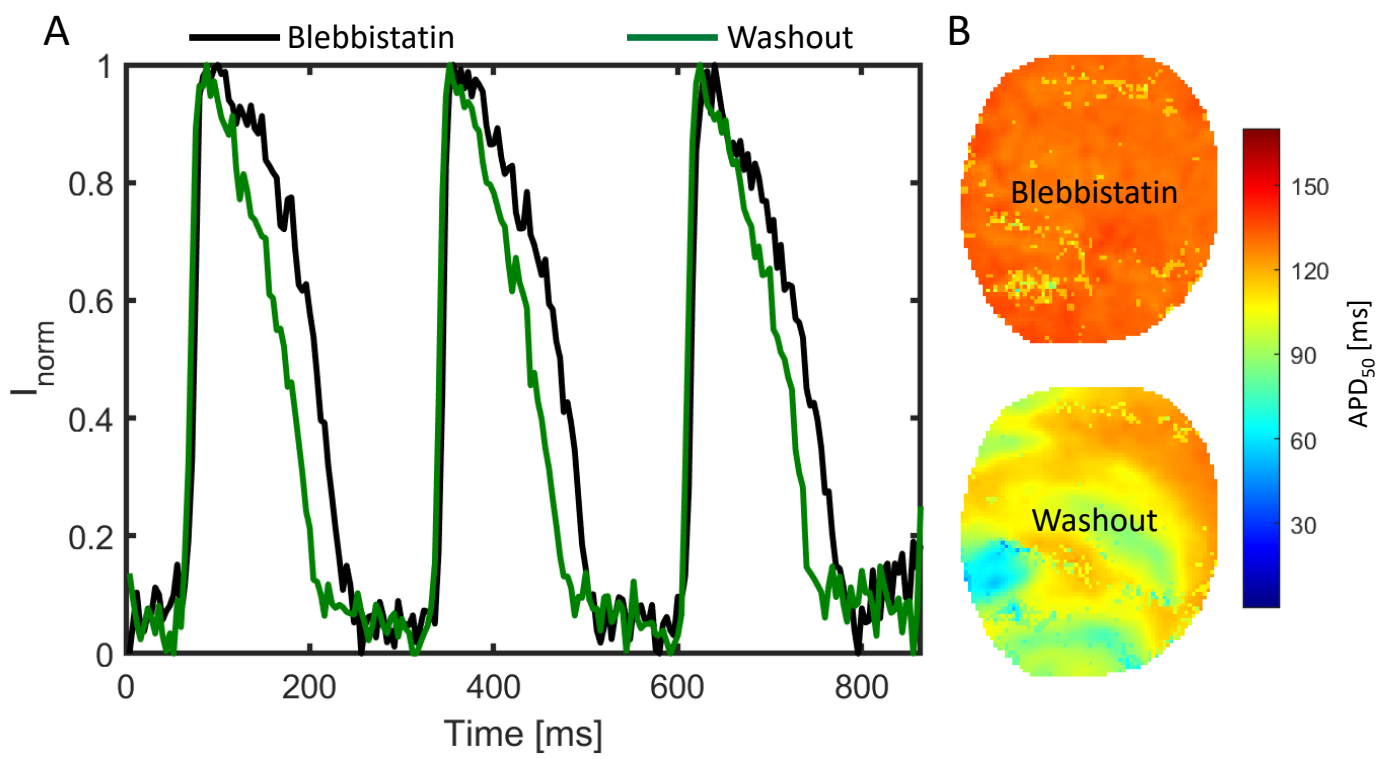

Fig. 5.10: Changes in action potential duration with washout of Blebbistatin. A) Comparison of action potential time traces obtained from $5 \times 5$ pixels from the left ventricular surface of a rabbit heart in Blebbistatin-uncoupled (black) and Blebbistatin-washout (green) conditions. Action potential durations shorten with the washout of Blebbistatin. B) APD maps showing spatial variation of action potential durations $\left(\mathrm{APD}_{50}\right)$ across the left ventricular surface in Blebbistatin-uncoupled (upper row) and Blebbistatinwashout (lower row) conditions. APD map with washout shows shortening of action potential durations for all pixels (representing the left ventricular surface) in comparison with Blebbistatin condition. 
Figure 5.10 shows the comparison of action potential durations in Blebbistatin-uncoupled and Blebbistatin-washout conditions. Panel A shows time traces (spatial averaged from $5 \times 5$ pixels) from the left ventricular surface of a rabbit heart in Blebbistatin-uncoupled (black; heart is not contracting) and Blebbistatin-washout (green; heart is contracting) conditions. The high spatial smoothing was used due to low signal to noise ratio of the optical action potential traces after the long waiting time (washout time $\approx 100$ minutes) during the experiment. The action potential duration decreases with Blebbistatin washout. Panel B shows the APD maps in Blebbistatin-uncoupled (upper row) and Blebbistatin-washout (lower row) conditions. The APD map in Blebbistatin-washout condition shows shortening of $\mathrm{APD}_{50}$ for all the pixels as compared to APD map of Blebbistatin-uncoupled conditions. This result is consistent with the findings of Figure 5.6, where action potential duration shows prolongation with Blebbistatin as compared to contracting condition. In short, administration of Blebbistatin to a contracting heart prolongs its action potential duration. APD comes back to shorter values upon washing out of the Blebbistatin. This implies that Blebbistatin-dependent APD changes are reversible.

\subsection{Impact of pacing frequency on electromechanical coupling}

To determine how the electrical activity affects the mechanical properties of the hearts, ratiometric optical mapping as described in section 3.2 was performed in contracting rabbit hearts during electrical pacing. The action potential time traces were extracted from the ratio video images of motion stabilized hearts (with motion tracking). The motion tracked data of either blue or green elicited action potentials were used to calculate motion amplitude as described in section 5.4. Figure 5.11 shows time traces of action potentials (green) and motion amplitudes (red) and the corresponding power spectra (calculated using Fourier transform of the time traces; green for action potential and red for motion amplitude) of both these traces for eight different pacing cycle lengths.

The action potentials and motion amplitudes were spatially averaged over all pixels for each cycle length. The panels A-H show the action potentials, 
A
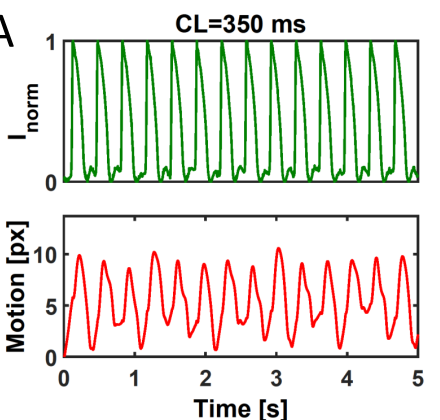

B
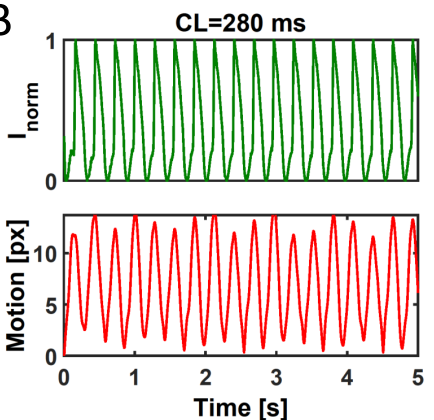

C
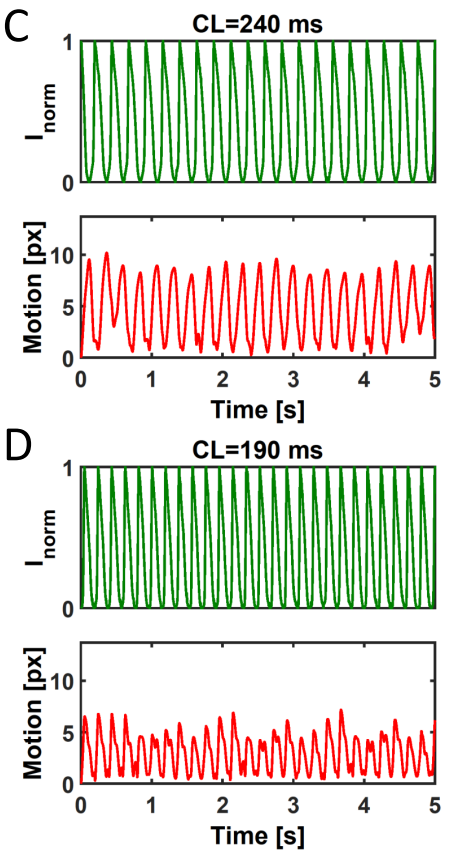

E
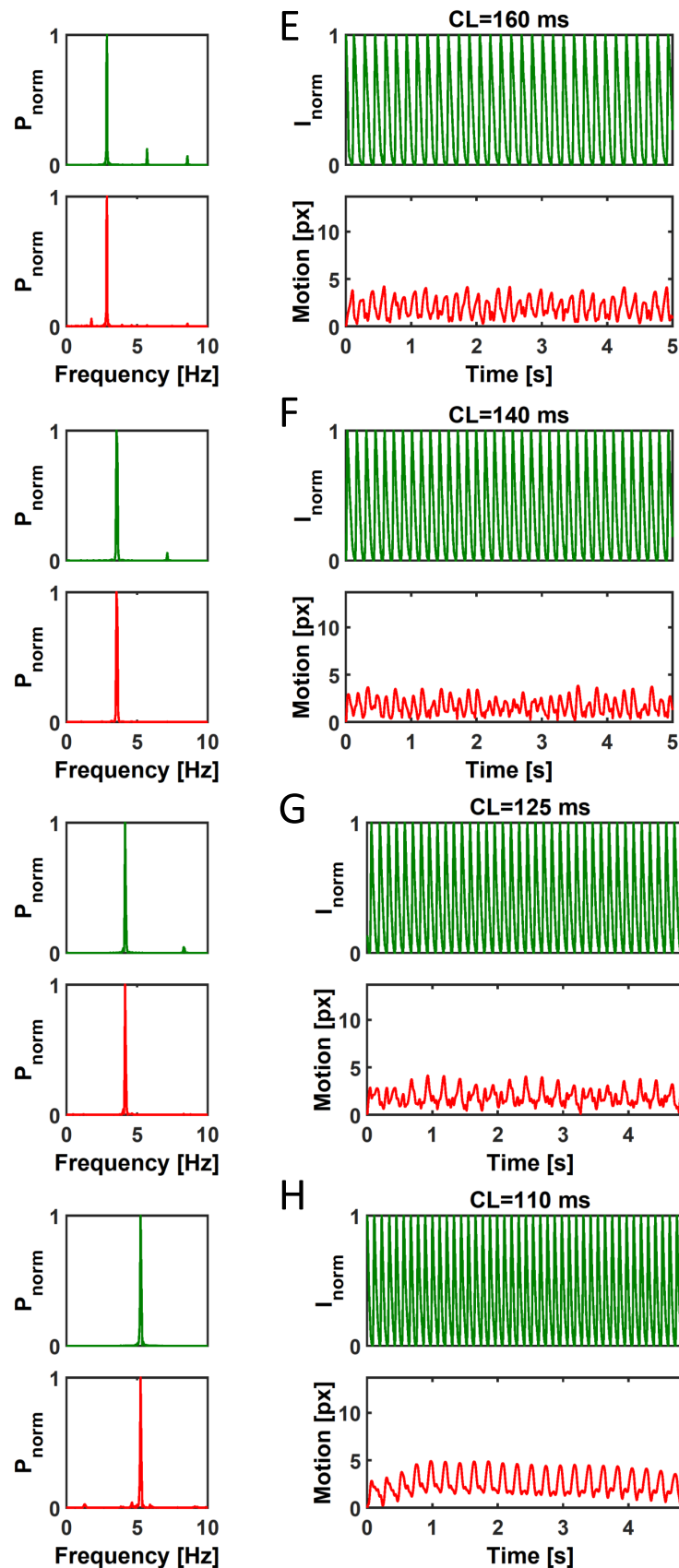

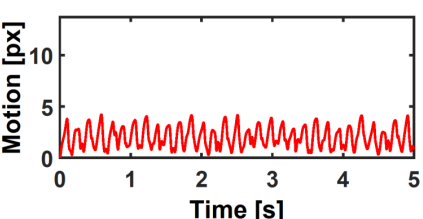

$\mathrm{F}$
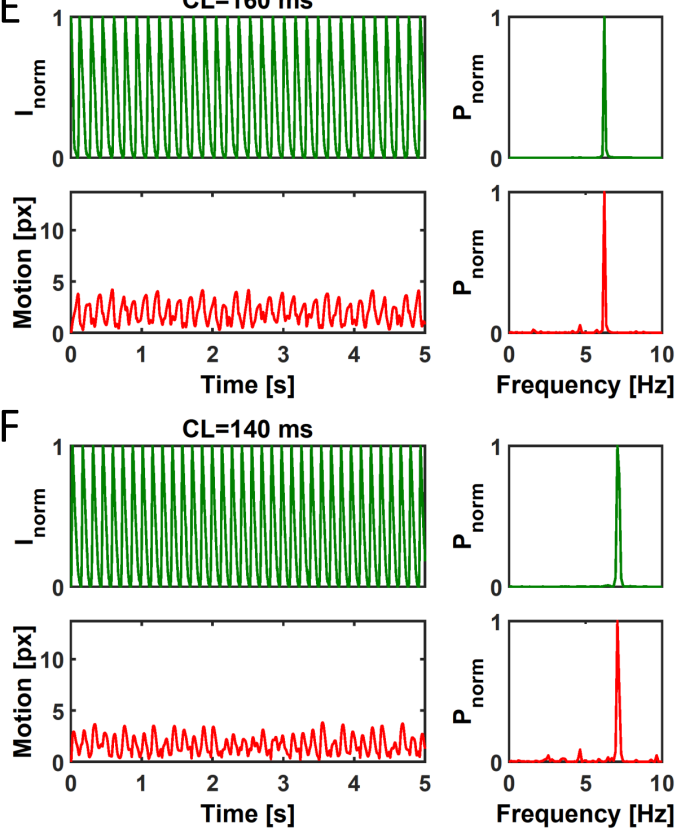

G
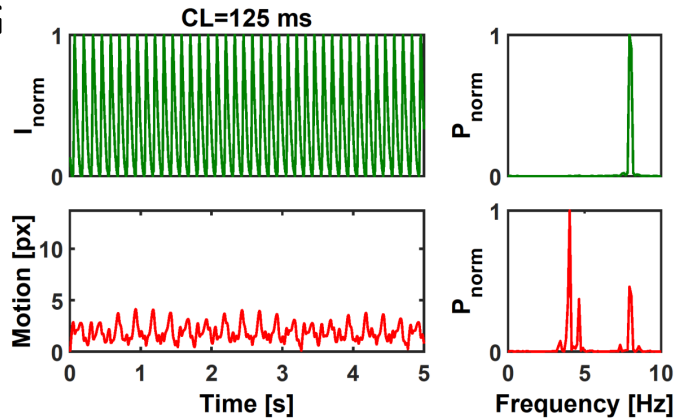

$\mathrm{H}$
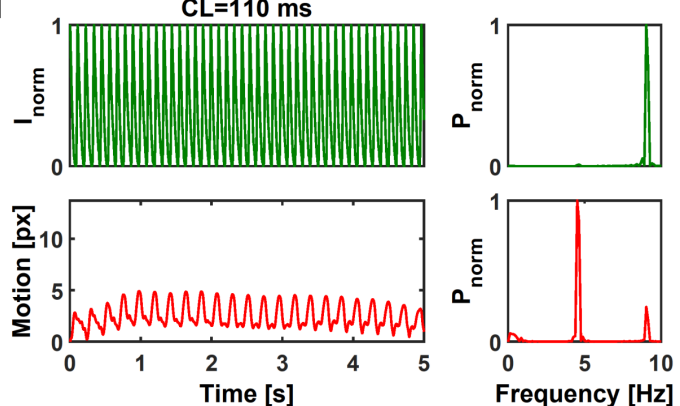

Fig. 5.11: Effect of pacing frequency on the electromechanical properties of the heart. Time series and corresponding power spectra of action potential (green) and motion amplitude (red) for different pacing frequencies (or pacing cycle length $C L$ ). Time series of action potential and motion amplitude were spatially averaged over all pixels representing the cardiac tissue. A) Time series and power spectra of action potential and motion amplitude for $C L=350 \mathrm{~ms}$ (pacing frequency $f_{p}=2.86 \mathrm{~Hz}$ ) B) Corresponds to $C L=280 \mathrm{~ms}$ (pacing frequency $f_{p}=3.57 \mathrm{~Hz}$ ) C) For $C L=240 \mathrm{~ms}$ (pacing frequency $f_{p}=4.17 \mathrm{~Hz}$ ) D) For $C L=190 \mathrm{~ms}$ (pacing frequency $f_{p}=5.26$ $\mathrm{Hz}$ ) E) For $C L=160 \mathrm{~ms}$ (pacing frequency $f_{p}=6.25 \mathrm{~Hz}$ ) F) For $C L=140$ $m s$ (pacing frequency $f_{p}=7.14 \mathrm{~Hz}$ ) G) For $C L=125 \mathrm{~ms}$ (pacing frequency $\left.f_{p}=8 \mathrm{~Hz}\right) \mathrm{H}$ ) For $C L=110 \mathrm{~ms}$ (pacing frequency $f_{p}=9.09 \mathrm{~Hz}$ ) 
motion amplitudes and their power spectra for cycle lengths $C L=350 \mathrm{~ms}$, $C L=280 \mathrm{~ms}, C L=240 \mathrm{~ms}, C L=190 \mathrm{~ms}, C L=160 \mathrm{~ms}, C L=140 \mathrm{~ms}, C L=125 \mathrm{~ms}$ and $C L=110 \mathrm{~ms}$, respectively. Action potential and motion amplitude are well coupled for all lower frequency stimulation until $C L=140 \mathrm{~ms}$ (pacing frequency $f_{p}=7.14 \mathrm{~Hz}$ ) as indicated by the identical frequencies in the power spectra of these electromechanical activities. For pacing frequencies higher than $7.14 \mathrm{~Hz}$, the action potential and motion amplitude time traces are not well coupled which results in the shift of the dominant frequency of the motion amplitude from that of the action potential for pacing frequencies values of $f_{p}=8 \mathrm{~Hz}(C L=125 \mathrm{~ms})$ and $f_{p}=9.09 \mathrm{~Hz}(C L=110 \mathrm{~ms})$. 


\section{Comparison of}

\section{ventricular fibrillation}

dynamics in

contracting and

Blebbistatin-uncoupled

\section{hearts}

An important method to characterize lethal ventricular fibrillation (VF) is by calculating the dominant frequency of electrical wave propagation using FFT (Fast Fourier Transform) based Fourier domain power spectra analysis. The dominant frequency of VF is usually defined as the largest peak in the FFT spectrum of the optical action potential signal [Val+02]. However, VF is chaotic and the FFT spectrum contains more frequency components with significant spectral powers in them, in addition to the dominant peak. Hence, selecting the dominant peak alone to calculate VF frequency is questionable. This chapter addresses the issue of dominant frequency calculation by using a weighted dominant frequency approach instead of dominant frequency itself. The weighted dominant frequencies were calculated for $N=10$ rabbit hearts in contracting and in non-contracting (with Blebbistatin) conditions and a systematic comparison of frequencies in both these conditions is also shown. Motion tracking and ratiometric measurements were used to extract the action potential time traces from contracting hearts during ventricular fibrillation. Ratiometric measurements were used in all Blebbistatin uncoupledconditions and the combined motion tracking and ratiometry were applied in hearts having slight movements even after Blebbistatin administration $(N=1)$. 


\subsection{Induction of ventricular fibrillation}

Ventricular fibrillation was often induced as a result of a dynamic pacing protocol with a local pacing electrode as described in section 5.1.1. This protocol was used for VF induction in $N=7$ hearts. For $N=3$ hearts, VF was induced by pacing the heart with the same local pacing electrode by applying high frequency (usually 30-50 $\mathrm{Hz}$ ) electrical pulses for $10-30$ seconds. Unsuccessful inductions were repeated either with the same frequency electrical pulses or with a slightly different value of frequency until fibrillation was induced.

\subsection{Dominant and weighted dominant frequen- cies of ventricular fibrillation}

To determine the dominant frequency of the hearts during ventricular fibrillation, action potential time traces were extracted from contracting and Blebbistatin-uncoupled hearts. The dominant frequencies were calculated for all the pixels (representing the left ventricle) as the frequencies corresponding to the largest peak in the action potential power spectra. Pixel wise dominant frequencies were used to construct dominant frequency maps.

Figure 6.1 shows dominant frequency maps and Fourier power spectra of optical action potential time traces during ventricular fibrillation across the left ventricular tissue in contracting and Blebbistatin-uncoupled conditions. The dominant frequency for each pixel was calculated as the frequency corresponding to the largest peak of the power spectrum. Panel A shows the dominant frequency map in contracting condition calculated from optical action potentials of 8 second duration. The dominant frequency map shows heterogeneity in dominant frequencies across the left ventricular tissue. Panel B shows optical action potential power spectrum in contracting condition for four different pixels (pixels represented by 1,2,3 and 4 in panel A) with dominant frequencies of $15.26 \mathrm{~Hz}, 13.43 \mathrm{~Hz}, 20.75 \mathrm{~Hz}$ and $9.88 \mathrm{~Hz}$, respectively.

Similarly, panel C shows the dominant frequency map and power spectra of the same heart after Blebbistatin administration. The dominant frequency map with Blebbistatin exhibits homogeneous distribution of dominant fre- 

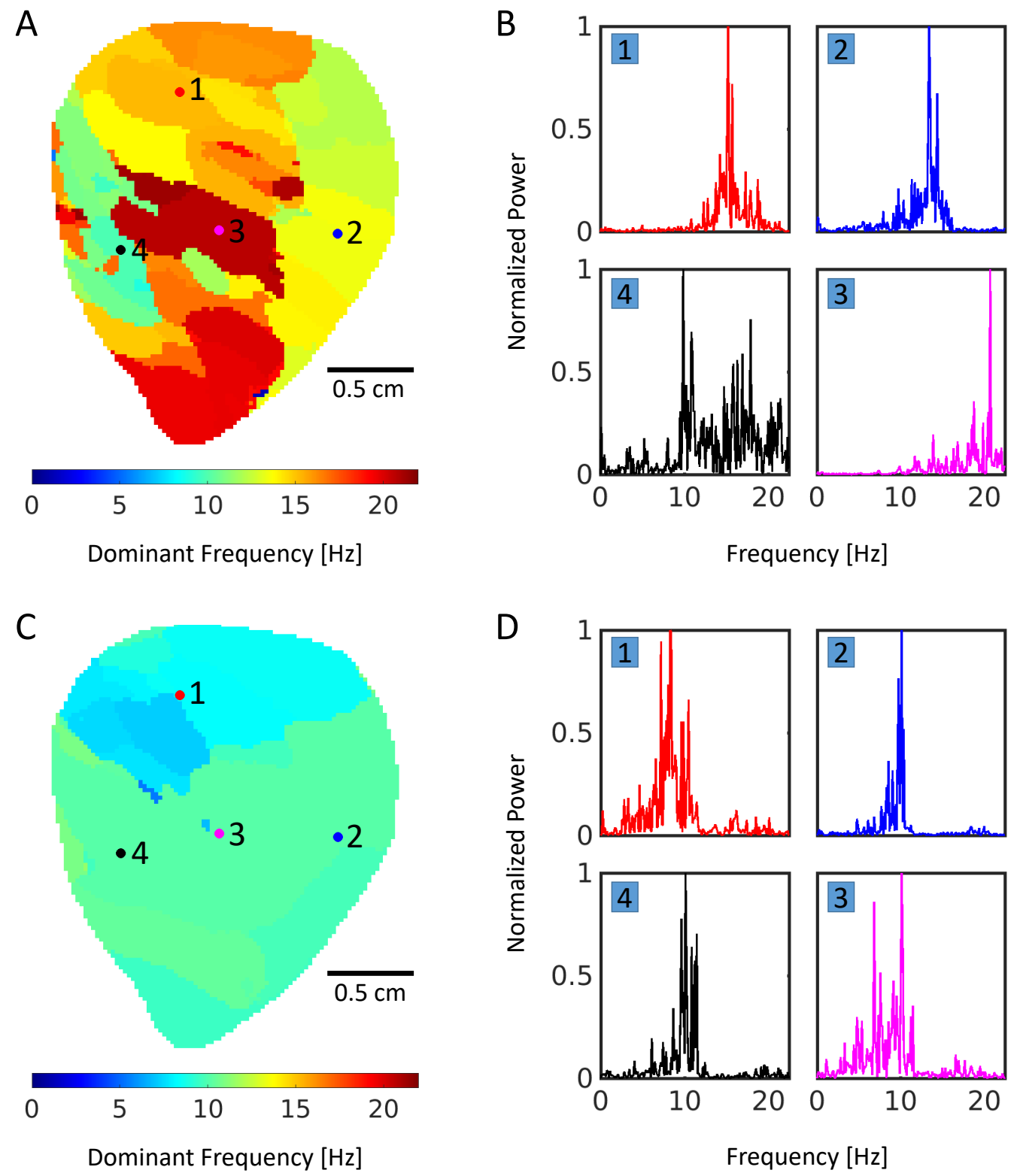

Fig. 6.1: Ventricular fibrillation frequency map and power spectra from a rabbit heart in contracting and Blebbistatin-uncoupled $(2.8 \mu \mathrm{M})$ conditions. A) Dominant frequency map showing dominant frequencies of action potential across the left ventricular heart tissue in contracting condition. The dominant frequency was calculated as the frequency corresponding to largest peak in the FFT spectrum. B) Optical action potential power spectra of four different pixels in contracting condition with dominant frequencies at $15.26 \mathrm{~Hz}, 13.43 \mathrm{~Hz}, 20.75 \mathrm{~Hz}$ and $9.88 \mathrm{~Hz}$, respectively. C) Dominant frequency map of VF in Blebbistatin-uncoupled condition D) Power spectra from four different pixels in Blebbistatin-uncoupled condition with dominant frequencies at $8.30 \mathrm{~Hz}, 10.13 \mathrm{~Hz}, 10.13 \mathrm{~Hz}$ and $10.13 \mathrm{~Hz}$, respectively. The power spectra and dominant frequencies were computed from optical action potential time traces of 8 second duration. 
quencies across the heart tissue as compared to the contracting condition. Power spectra of four different pixels are also partially similar with dominant frequency values of $8.30 \mathrm{~Hz}, 10.13 \mathrm{~Hz}, 10.13 \mathrm{~Hz}$ and $10.13 \mathrm{~Hz}$, respectively.

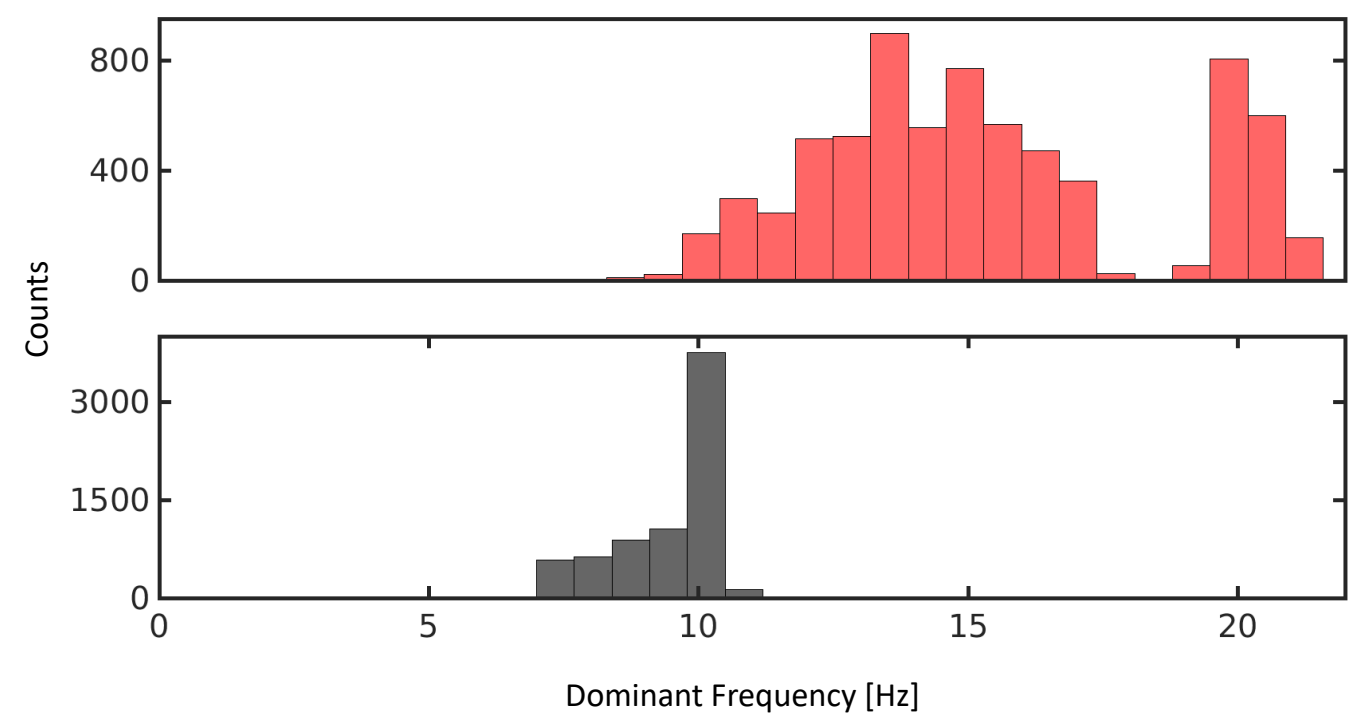

Fig. 6.2: Histogram of dominant frequency map showing dominant frequency of the rabbit heart during ventricular fibrillation in contracting (red) and in Blebbistatin (2.8 $\mu \mathrm{M}$;black) conditions. The dominant frequencies were calculated from dominant frequency maps.

Figure 6.2 shows the comparison of dominant frequencies of the heart in contracting and Blebbistatin-uncoupled conditions as a histogram (dominant frequencies were calculated from dominant frequency maps as shown in Figure 6.1A and Figure 6.1C). Significant reduction in dominant frequencies of ventricular fibrillation is noticeable in Blebbistatin administrated condition as compared to contracting condition. Also, dominant frequencies in contracting condition are spatially more dispersed in comparison with Blebbistatin-coupled condition.

The power spectra in Figure 6.1B and Figure 6.1D indicate that there is significant spectral power in frequency components in addition to the dominant peaks (see panel B4 and panel D3). Hence, selecting the largest peak of the power spectrum as the dominant frequency excludes information of other frequency components which have noticeable spectral power in the FFT spectra. In order to include the contribution of other frequency components, a weighted dominant frequency is defined and calculated for 
each power spectrum with the following formula

$$
f_{w d}=\frac{\sum_{i} f_{i} P_{i}}{\sum_{i} P_{i}}, \quad P_{i}>P_{t h}
$$

Where, $f_{i}$ represents the $\mathrm{i}^{\text {th }}$ frequency component in the power spectrum such that corresponding power $P_{i}$ is greater than the threshold value $P_{t h}$. The equation (6.1) is used to avoid biasing in selecting a single frequency when the dominant frequency is not predominant due to the presence of multiple frequency components with significant spectral powers.

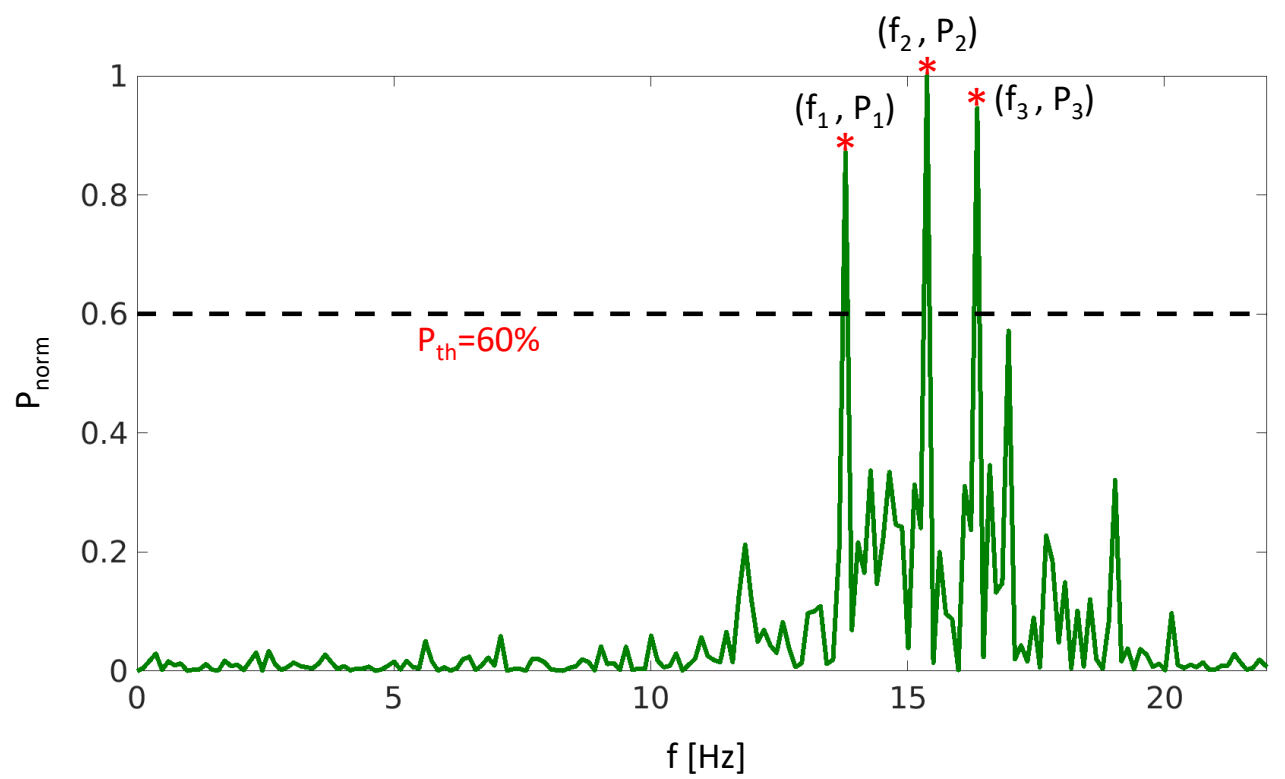

Fig. 6.3: Calculation of weighted dominant frequency. Example of a broadened power spectrum from a contracting rabbit heart during ventricular fibrillation. Weighted dominant frequency is calculated by using the powers greater than the threshold value $P_{t h}$ (60\% in this case) and the corresponding frequencies.

An example of a broadened FFT spectra (normalized) is shown in Figure 6.3. There are three frequency values $\left(f_{1}, f_{2}\right.$ and $\left.f_{3}\right)$ having spectral powers $\left(P_{1}, P_{2}\right.$ and $\left.P_{3}\right)$ higher than the threshold $P_{t h}$. In this example, selecting a single dominant frequency $\left(f_{2}\right)$ completely ignores the contributions of $f_{1}$ and $f_{3}$ even though they contain noticeable spectral powers. The power threshold is kept at $60 \%$ of the maximum power $\left(P_{\max }=1\right.$, due to normalization) only to demonstrate the calculation of weighted dominant frequency. However, this threshold can, in theory, take any other values in the range 
$0-100 \%$. In order to see the effect of the power threshold on the weighted dominant frequency, five different threshold values such as $70 \%, 50 \%, 30 \%$, $10 \%$ and $0 \%$ of the maximum power were used. The weighted dominant frequency is calculated by weighting the frequency components which are above the power threshold using equation (6.1).

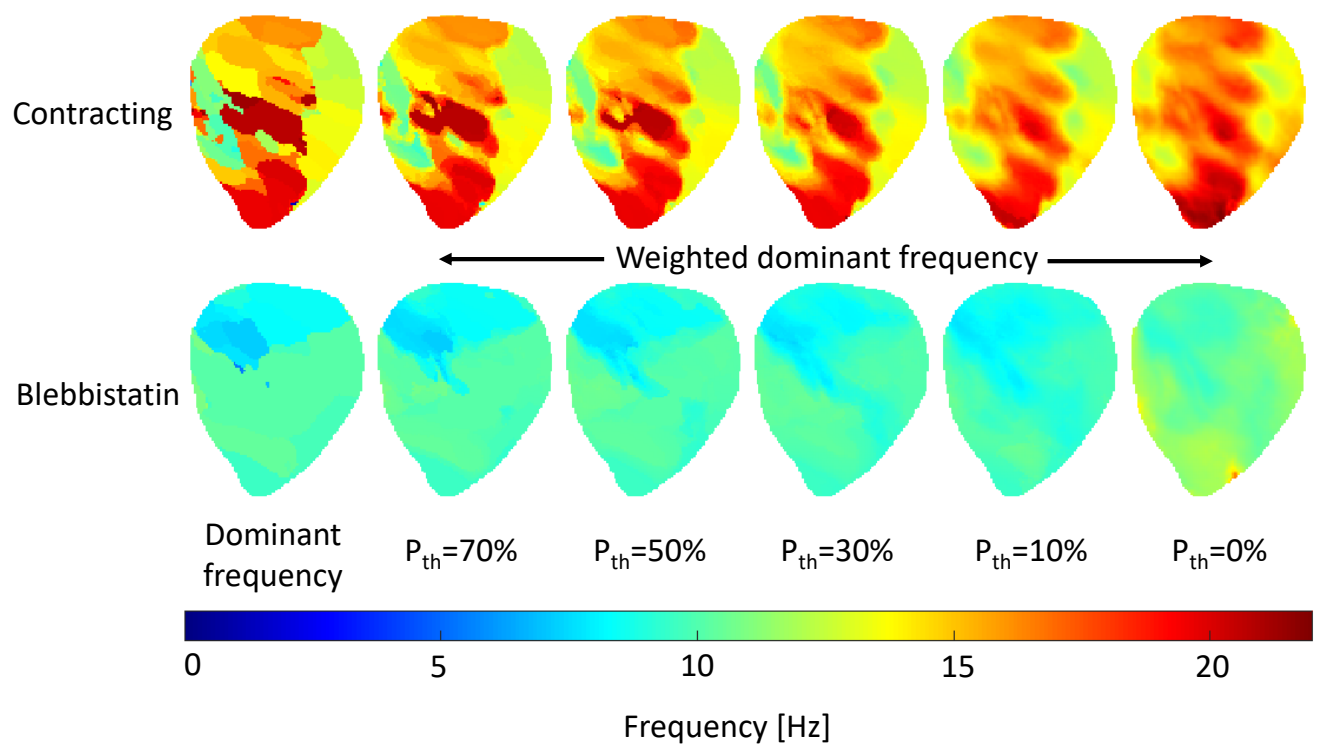

Fig. 6.4: Frequency maps showing weighted dominant frequencies of a rabbit heart in contracting (upper row; with motion stabilization and ratiometry) and Blebbistatin-uncoupled (lower row; $2.8 \mu \mathrm{M}$ ) conditions. Frequency maps from left to right (except the first left column) show the variation of weighted dominant frequencies with different power thresholds $\left(P_{t h}\right)$. Weighted dominant frequency is calculated by weighting the frequency components which are above the threshold value in the power spectrum. The frequency maps on the first left column (Dominant frequency) show the spatial variation of dominant frequency, calculated by selecting the largest peak in the power spectrum without giving weight to other frequency components.

Figure 6.4 illustrates the change in frequency maps of ventricular fibrillation with different power threshold in contracting and Blebbistatin-uncoupled $(2.8 \mu \mathrm{M})$ conditions for a single heart (same heart used in Figure 6.1). Dominant frequency maps (left raw; dominant frequency) were calculated by selecting the largest peaks in the power spectra without giving any weight to other frequency components and they are same as the frequency maps shown in Figure 6.1A and Figure 6.1C. Weighted dominant frequency maps with different power threshold display slightly different frequency values and 
the frequency maps undergo smoothing behavior as we decreases the power threshold. The small rise in frequency values with a power threshold of $0 \%$ is due to the presence of high frequency noise values while weighting all the frequency components within the power spectrum. However, the difference in the dominant frequencies of the heart in contracting and Blebbistatinuncoupled conditions is still consistent even after giving weight to other frequency components in the power spectrum.

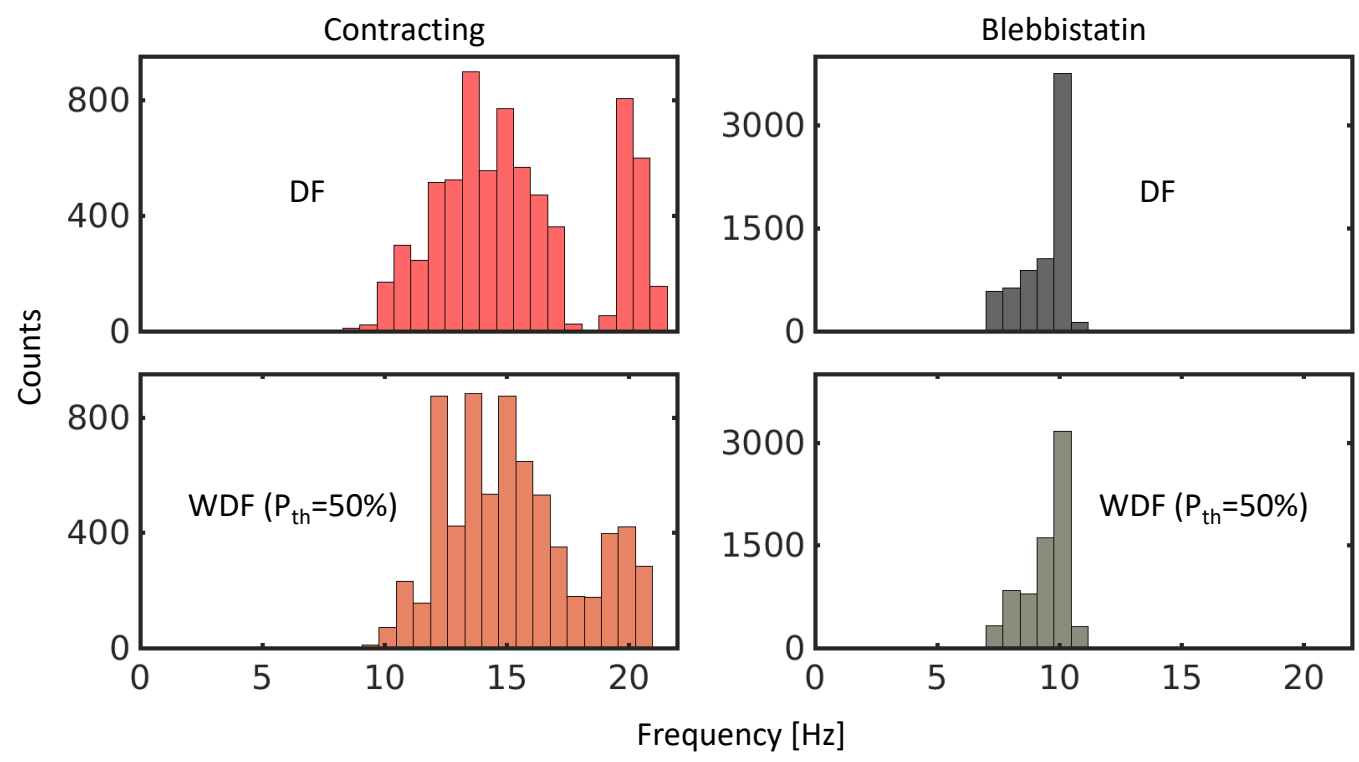

Fig. 6.5: Histogram showing dominant frequencies of a single rabbit heart during ventricular fibrillation in contracting (left: red and brown) and in $2.8 \mu \mathrm{M}$ Blebbistatin (right: black and gray) conditions. Upper raw (red and black) corresponds to dominant frequencies (DF) obtained from the largest peaks of the power spectra and lower raw shows weighted dominant frequencies (WDF) obtained from multiple peaks with a power threshold $\left(P_{t h}\right)$ of $50 \%$ (brown and gray).

The same effect is also salient in the histogram shown in Figure 6.5, where the dominant frequency (DF) and weighted dominant frequencies (WDF at $50 \%$ power threshold) are within $10-20 \mathrm{~Hz}$ in contracting condition and are around $10 \mathrm{~Hz}$ for Blebbistatin-uncoupled condition. Hence, it confirms that, the observed effect in the dominant frequency is due to the administration of Blebbistatin and not due to any bias in frequency calculation.

Figure 6.6 shows the variation of weighted dominant frequencies (except the first two bars on the left) in contracting and Blebbistatin-uncoupled conditions for different power thresholds. The first two bars represent 


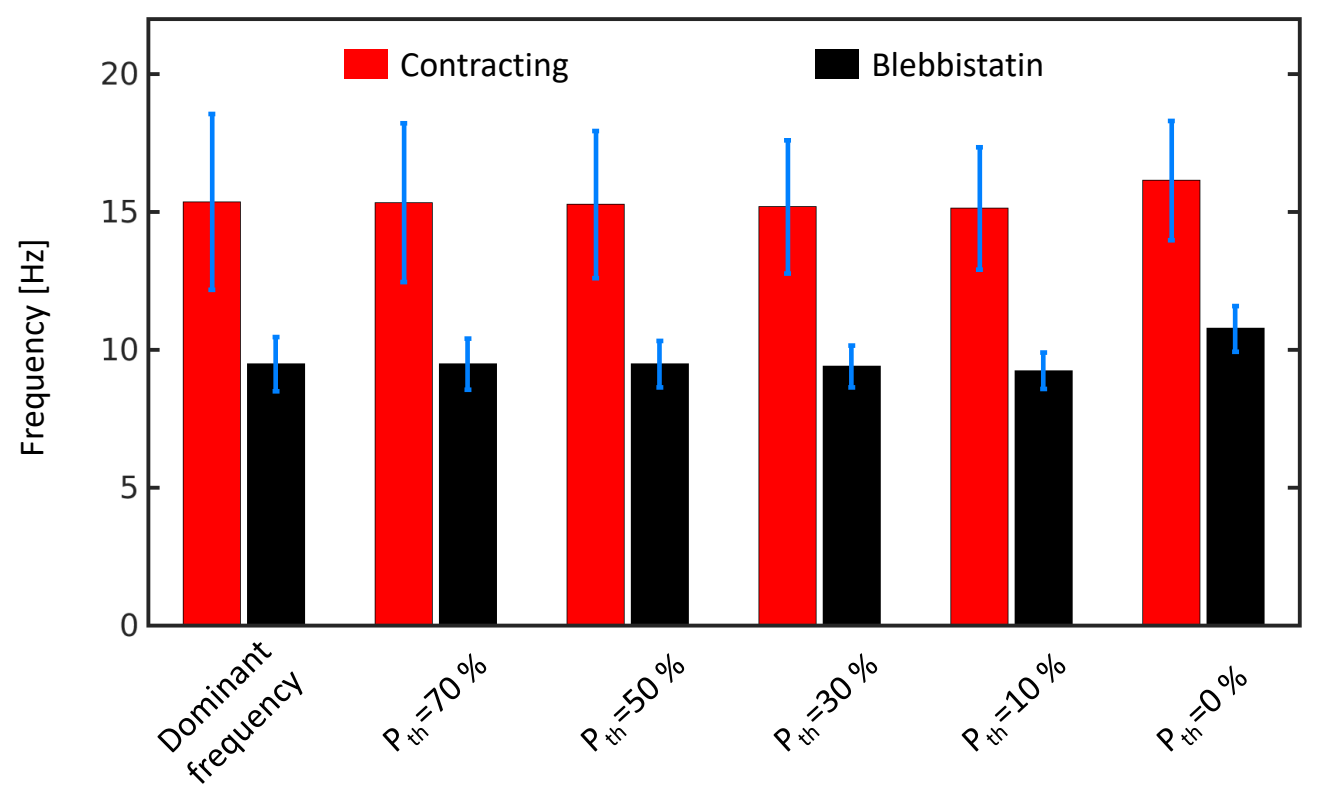

Fig. 6.6: Bar plot showing dominant frequencies of ventricular fibrillation from a contracting (red) and Blebbistatin-uncoupled (black) rabbit heart for different power thresholds. Error bars show the standard deviation of VF frequencies calculated from the frequency maps shown in Figure 6.4.

dominant frequencies in contracting and Blebbistatin conditions, respectively. The bar plots represent the mean and standard deviation of VF frequencies calculated from the frequency maps shown in Figure 6.4. The frequencies of VF in contracting condition are $15.36 \pm 3.19 \mathrm{~Hz}, 15.34 \pm 2.87 \mathrm{~Hz}, 15.27 \pm 2.66$ $\mathrm{Hz}, 15.19 \pm 2.41 \mathrm{~Hz}, 15.14 \pm 2.22 \mathrm{~Hz}$ and $16.14 \pm 2.16 \mathrm{~Hz}$ corresponding to the frequency calculation methods such as dominant frequency, $P_{t h}=70 \%$, $P_{t h}=50 \%, P_{t h}=30 \%, P_{t h}=10 \%$ and $P_{t h}=0 \%$, respectively. Similarly, the frequency values in Blebbistatin condition are $9.48 \pm 0.99 \mathrm{~Hz}, 9.47 \pm 0.92 \mathrm{~Hz}$, $9.48 \pm 0.84 \mathrm{~Hz}, 9.41 \pm 0.76 \mathrm{~Hz}, 9.24 \pm 0.65 \mathrm{~Hz}$ and $10.77 \pm 0.83 \mathrm{~Hz}$ corresponding to dominant frequency, $P_{t h}=70 \%, P_{t h}=50 \%, P_{t h}=30 \%, P_{t h}=10 \%$ and $P_{t h}=0 \%$, respectively. The mean and standard deviation of frequencies on average decreases as we go from dominant frequency to $P_{t h}=10 \%$ due to the inclusion of lower frequency components from the power spectra in addition to the largest peak. For $P_{t h}=0 \%$, all the frequency values and the corresponding power in the power spectra are used to calculate weighted dominant frequency. The weighted dominant frequency in this case shows an increase in the mean value due to the presence of high frequency noise in the power spectra. 


\subsection{Variation of ventricular fibrillation frequen- cies with Blebbistatin concentration}

Variation of ventricular fibrillation frequencies with different Blebbistatin concentrations (0-2.8 $\mu \mathrm{M})$ was investigated and the frequency maps containing dominant (upper row) and weighted dominant frequencies (lower row; $\left.P_{t h}=50 \%\right)$ are shown in Figure 6.7.

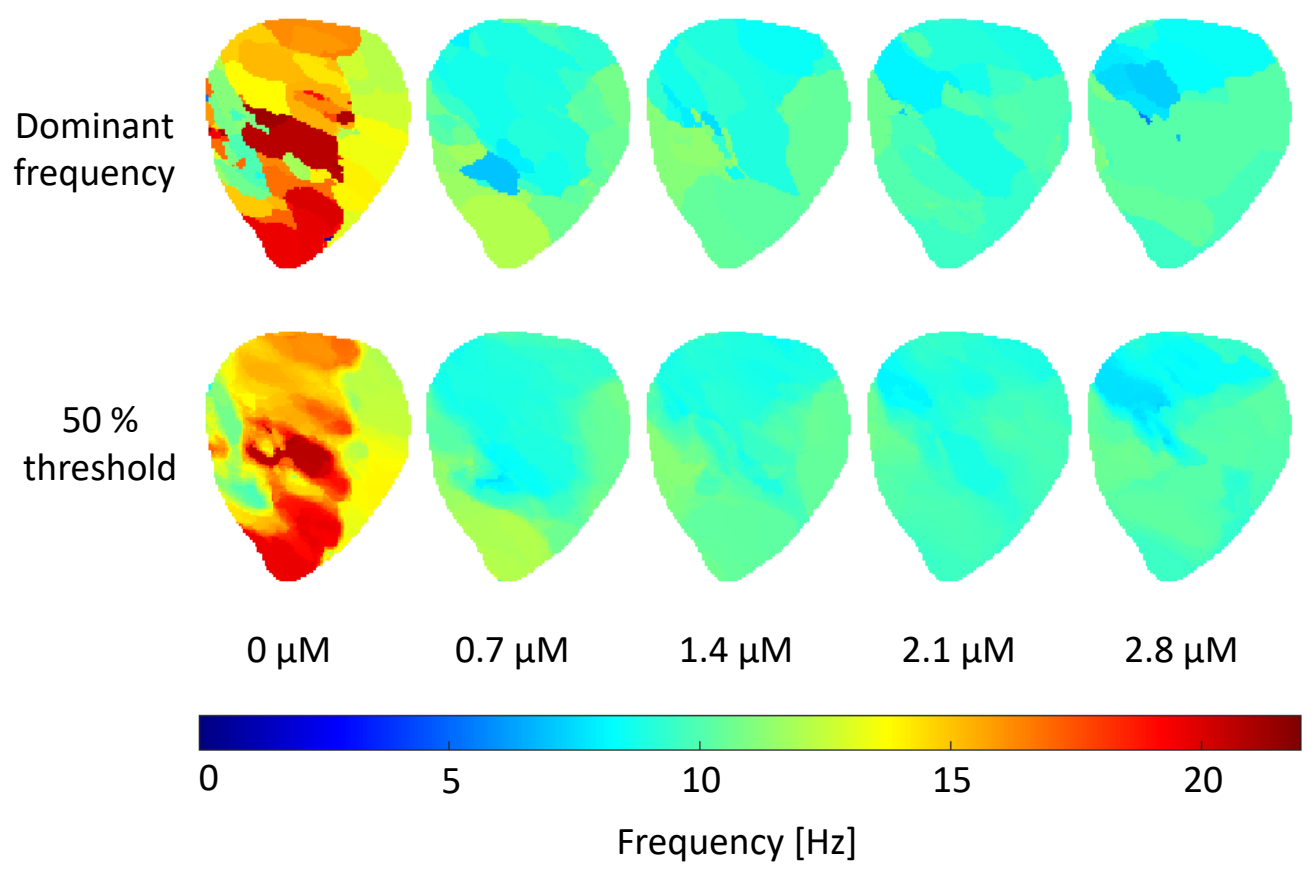

Fig. 6.7: Frequency maps showing the variation of ventricular fibrillation frequency with Blebbistatin concentration. Upper row shows the dominant frequency maps calculated from the largest peaks of the power spectra and lower row corresponds to weighted dominant frequency calculated from multiple peaks with $50 \%$ power threshold $\left(P_{t h}\right)$. Significant change in dominant frequency is observed with $0.7 \mu \mathrm{M}$ of Blebbistatin concentration, whereas the changes in frequencies are not very significant with higher concentration.

From $0 \mu \mathrm{M}$ to $0.7 \mu \mathrm{M}$ of Blebbistatin, the maximum change in VF frequency is observed. After that, slight changes in frequencies (dominant frequency from $9.74 \mathrm{~Hz}$ to $9.48 \mathrm{~Hz}$, weighted dominant frequency from $9.80 \mathrm{~Hz}$ to 9.48 $\mathrm{Hz}$ ) is noticed with the increase in Blebbistatin concentration from $0.7 \mu \mathrm{M}$ to $2.8 \mu \mathrm{M}$.

The bar plots in Figure 6.8 display the change in ventricular fibrillation frequencies (mean and standard deviation calculated from frequency maps) 


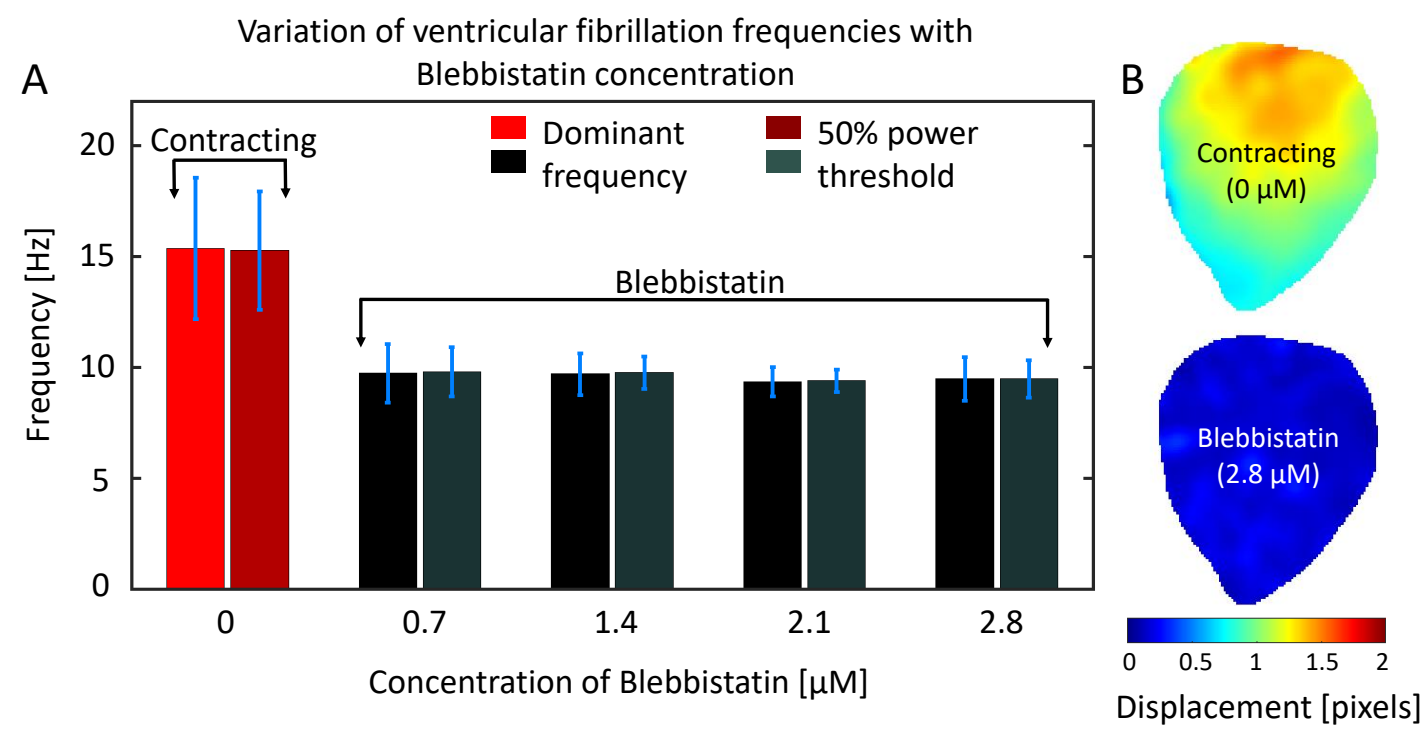

Fig. 6.8: Ventricular fibrillation dynamics with Blebbistatin concentration. A) Bar plots showing the mean and standard deviation of VF frequencies calculated with dominant frequency (red and black) and weighted dominant frequency (50\% power threshold; reddish brown and dark green) approaches for different Blebbistatin concentrations (0, 0.7, 1.4, 2.1 and 2.8 $\mu \mathrm{M})$. Error bars represent the standard deviation of dominant frequencies from multiple pixels along the left ventricle (calculated from frequency maps shown in Figure 6.7). B) Spatial variation of displacement (in pixels) of the heart during ventricular fibrillation in fully contracting $(0 \mu \mathrm{M})$ and Blebbistatin $(2.8 \mu \mathrm{M})$ conditions.

for different Blebbistatin concentrations such as $0 \mu \mathrm{M}, 0.7 \mu \mathrm{M}, 1.4 \mu \mathrm{M}$, $2.1 \mu \mathrm{M}$ and $2.8 \mu \mathrm{M}$. Blebbistatin concentration of $0 \mu \mathrm{M}$ represents a fully contracting heart with an average displacement of 1.13 pixels. At $2.8 \mu \mathrm{M}$, the displacement of the heart becomes negligible (average displacement= 0.16 pixel). Adding $0.7 \mu \mathrm{M}$ of Blebbistatin to the contracting heart produced significant decrease $(\approx 35 \%)$ in dominant and weighted dominant frequencies. Increasing the concentration of Blebbistatin further decreases the frequencies slightly. The bar plots show dominant frequencies values of $15.36 \pm 3.19 \mathrm{~Hz}, 9.74 \pm 1.32 \mathrm{~Hz}, 9.70 \pm 0.94 \mathrm{~Hz}, 9.35 \pm 0.65 \mathrm{~Hz}, 9.48 \pm 0.99 \mathrm{~Hz}$ corresponding to the Blebbistatin concentration of $0 \mu \mathrm{M}, 0.7 \mu \mathrm{M}, 1.4 \mu \mathrm{M}, 2.1$ $\mu \mathrm{M}$ and $2.8 \mu \mathrm{M}$, respectively. The weighted dominant frequencies $\left(P_{t h}=50 \%\right)$ for these concentrations are $15.27 \pm 2.66 \mathrm{~Hz}, 9.80 \pm 1.11 \mathrm{~Hz}, 9.76 \pm 0.73 \mathrm{~Hz}$, $9.40 \pm 0.51 \mathrm{~Hz}, 9.48 \pm 0.84 \mathrm{~Hz}$, respectively. 


\subsection{Ventricular fibrillation frequencies from multiple hearts}

All the frequency analysis of previous sections were carried out for a single heart (Heart 8 in Figure 6.9). This section focuses on the analysis of ventricular fibrillation frequencies for multiple hearts. The VF frequency measurements were repeated for $N=10$ rabbit hearts and the comparison of the frequencies (weighted dominant frequencies with $P_{t h}=50 \%$ ) in contracting and Blebbistatin-uncoupled conditions are shown in Figure 6.9.

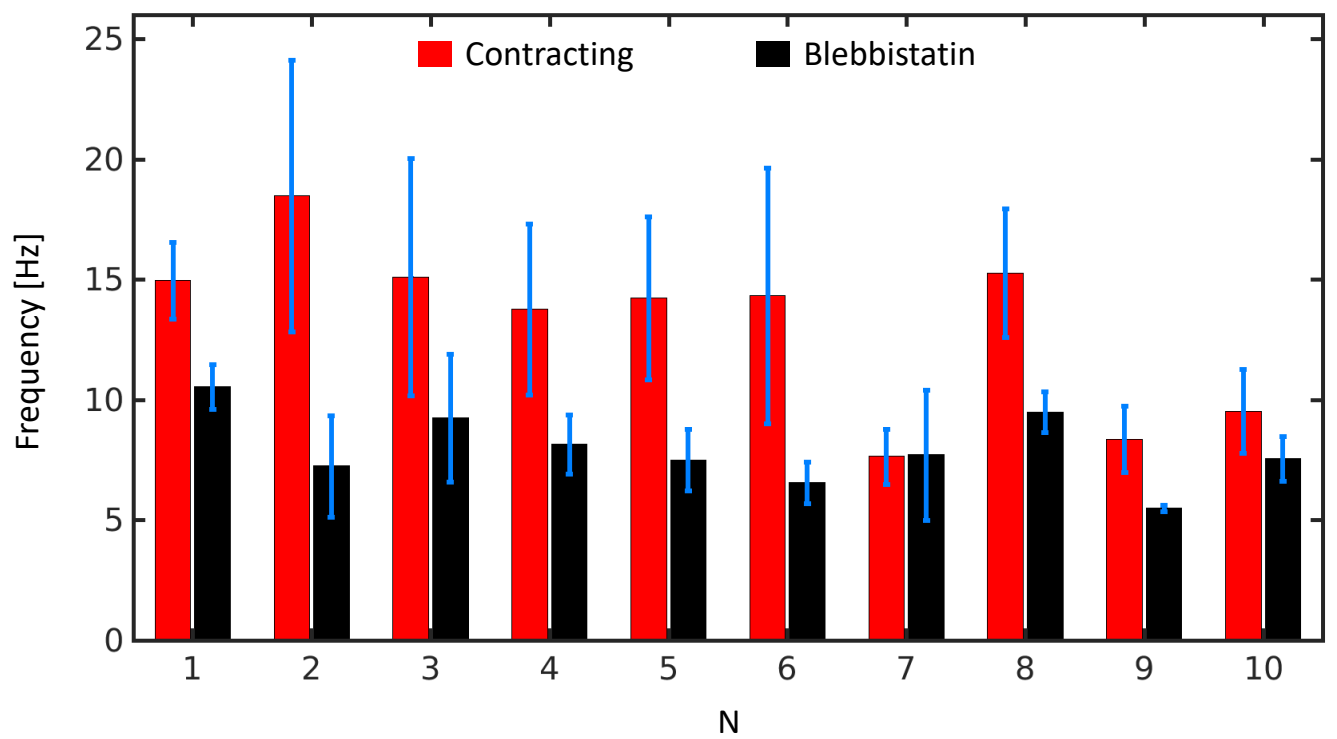

Fig. 6.9: Bar plot showing the comparison of weighted dominant frequencies $\left(P_{t h}=50 \%\right)$ of ventricular fibrillation for 10 hearts in contracting heart (red) and Blebbistatin-uncoupled (black) conditions. The error bars represent the standard deviation of frequencies calculated from frequency maps. The weighted dominant frequencies for each heart was calculated from time traces consisting of 8 seconds duration.

The bar plots show the mean and standard deviations of VF frequencies calculated from the corresponding frequency maps. VF was induced with dynamic pacing protocol in 7 hearts (hearts 2-8), whereas for 3 hearts (hearts $1,9,10) \mathrm{VF}$ induction was done by pacing with high frequency electrical pulses as mentioned in 6.1.

The Blebbistatin concentration for $N=8$ hearts was $5.6 \mu \mathrm{M}$ and the concentration for $N=2$ hearts (heart 8 and heart 9) was $2.8 \mu \mathrm{M}$, where the 


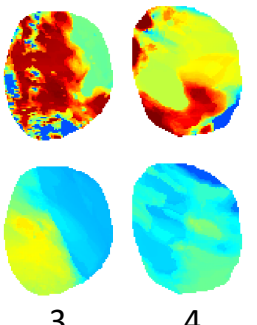

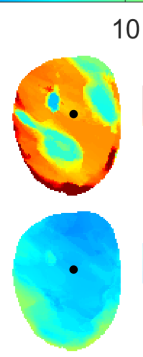

5

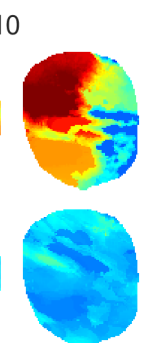

6

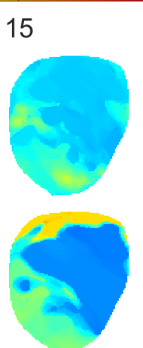

7

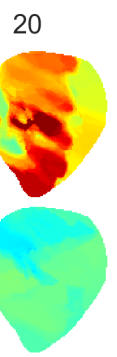

8

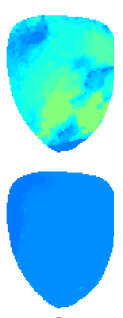

9

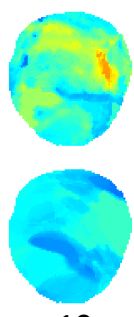

10
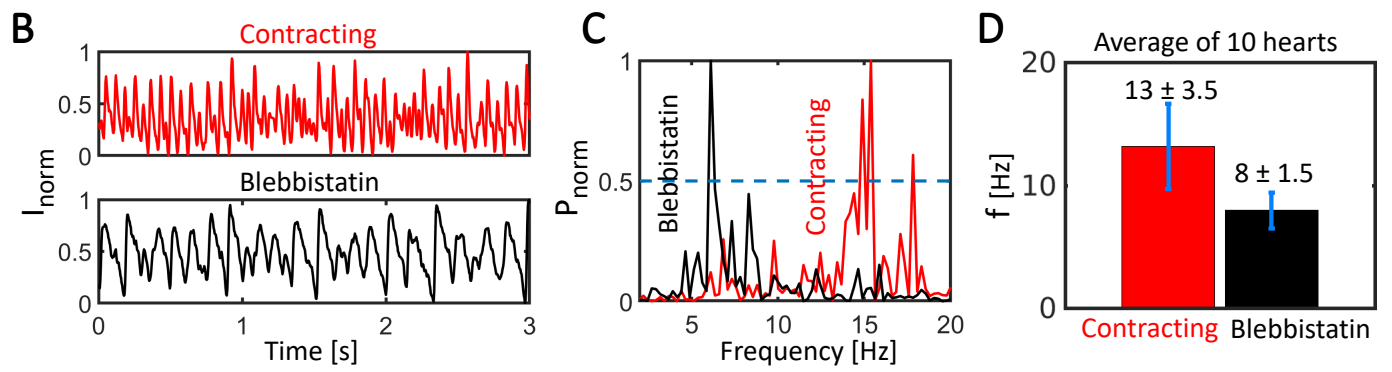

Fig. 6.10: Weighted dominant frequency comparison $\left(P_{t h}=50 \%\right)$ between contracting and Blebbistatin-uncoupled hearts during ventricular fibrillation. A) Frequency maps (for $N=10$ hearts) of left ventricular tissue showing higher frequency of ventricular fibrillation in contracting hearts as compared to Blebbistatin-uncoupled conditions. B) Time series of VF from heart 5 (shown only for 3 seconds). Time traces are extracted from the pixel shown in the center (as a black dot) after spatio-temporal averaging the video frames with a window of size $3 \times 3 \times 3$. C) Power spectrum calculated from panel B (but with time traces of 8 seconds duration with 2000 samples). D) Bar plot showing the average dominant frequency from 10 hearts indicating significant variation of VF frequencies in contracting versus Blebbistatin-uncoupled hearts.

concentration was progressively increased until $2.8 \mu \mathrm{M}$ to investigate the concentration dependency of VF frequencies. All hearts except heart 7 show decrease in VF frequencies in Blebbistatin-uncoupled condition as compared to contracting condition. Additionally, the standard deviation of frequencies in contracting condition for these $N=9$ hearts are higher than in the Blebbistatin case, indicating high spatial dispersion of frequencies across the left ventricle during contraction. Heart 7 does not show changes in mean value of frequencies in contracting and Blebbistatin conditions. But it is to be noted that unlike all other hearts, the standard deviation of frequencies in Blebbistatin condition is higher than in contracting condition. On average, the VF frequencies in contracting and Blebbistatin conditions are $13 \pm 3.5$ and $8 \pm 1.5$, respectively. Hence, VF dynamics in contracting condition are 
characterized by higher frequency of electrical excitation as compared to Blebbistatin-uncoupled conditions irrespective of the protocol used to induce VF.

Figure 6.10 shows a more detailed analysis of VF dynamics in contracting and Blebbistatin-uncoupled conditions. Panel A shows the frequency maps for $N=10$ hearts in these conditions. Panel B shows exemplary time traces from heart 5 (time traces extracted from the pixels denoted by the black dot in the center). Panel C shows the frequency domain power spectrum calculated from the time traces shown in panel B. For frequency calculation 8 second duration time traces were used. The blue dashed lines at $P_{\text {norm }}=0.5$ shows 50\% power threshold used to calculate weighted dominant frequency. Panel D shows mean and standard deviations of VF frequencies calculated from all $N=10$ hearts. Panels A-D in general show accelerated VF dynamics in contracting hearts as compared to Blebbistatin-uncoupled hearts. In other words, administration of Blebbistatin reduced VF dynamics in 9 out of $N=10$ hearts. On average VF frequencies in contracting hearts are $62 \%$ higher than in Blebbistatin condition.

\subsection{Variation of ventricular fibrillation frequencies with Blebbistatin washout}

Similar to the observation in section 5.8, Blebbistatin washout was employed in $N=2$ rabbit hearts for 60-100 minutes. Figure 6.11 illustrates the comparison of optical action potential time traces in contracting (red), Blebbistatin-uncoupled (1.4 $\mu \mathrm{M})$; black) and Blebbistatin washout (green) conditions from a single heart (heart 9 of Figure 6.10). Similar to the findings of section 6.4, VF frequency of the contracting heart decreased from $\mathrm{WDF}=10.62 \mathrm{~Hz}$ to $\mathrm{WDF}=5.55 \mathrm{~Hz}$ with Blebbistatin-administration. After washing out the Blebbistatin for $\approx 100$ minutes, the VF frequency increased to $7.02 \mathrm{~Hz}$. This indicates reversibility of VF frequencies with Blebbistatin washout. However, the frequency value in washout condition lies in between frequencies of contracting and Blebbistatin-uncoupled conditions, suggesting that reversibility of VF dynamics is only partial. 

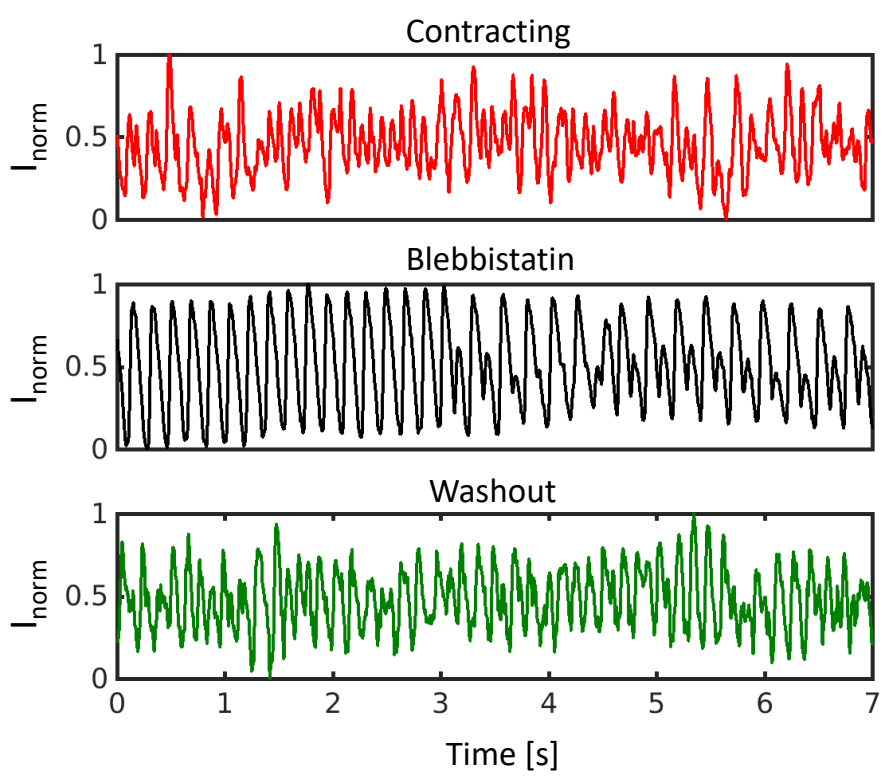
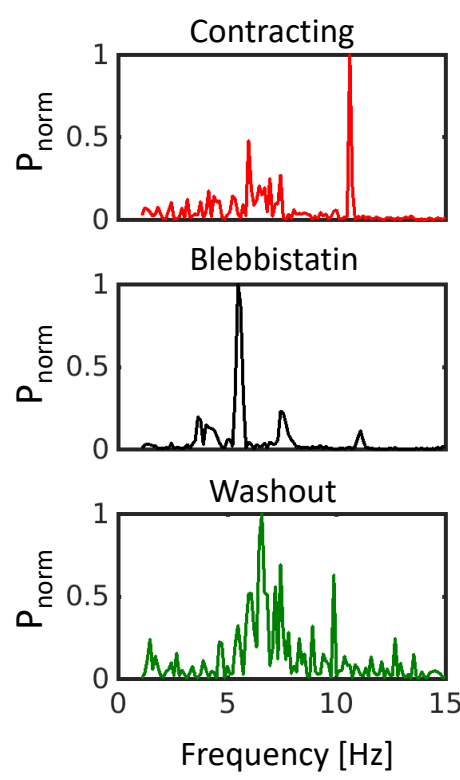

Fig. 6.11: Variation of VF frequencies with Blebbistatin washout. Optical action potential time traces during ventricular fibrillation in contracting, Blebbistatin-uncoupled (1.4 $\mu \mathrm{M})$ and Blebbistatin-washout conditions from a single heart. Irregular and high frequency VF activities become more regular and slower with Blebbistatin (induction of VF was very difficult with Blebbistatin). Upon washout of Blebbistatin for 100 minutes, an increase in VF frequency is observed with a frequency value that lies in between that of contracting and Blebbistatin-uncoupled conditions.

\subsection{Comparison of ventricular fibrillation frequencies calculated from ECG and optical data}

The robustness of the frequency measurements were examined by calculating the VF frequencies using optical action potential and ECG time traces for $N=2$ hearts (heart 5 and heart 8 of Figure 6.10). Figure 6.12 shows the comparison of Fourier power spectra from heart 5 (panel A) and heart 8 (panel B) using ECG (upper row) and optical action potential traces (lower row) in contracting (red) and Blebbistatin-uncoupled (black) conditions. Optical power spectra (power spectra from optical action potential traces) were calculated by averaging the Fourier power from all pixels (pixels used to create frequency maps). The green and blue dashed lines in the case of optical data represent mean values of weighted dominant frequencies 
in contracting and Blebbistatin conditions calculated from corresponding frequency maps (see Figure 6.10).
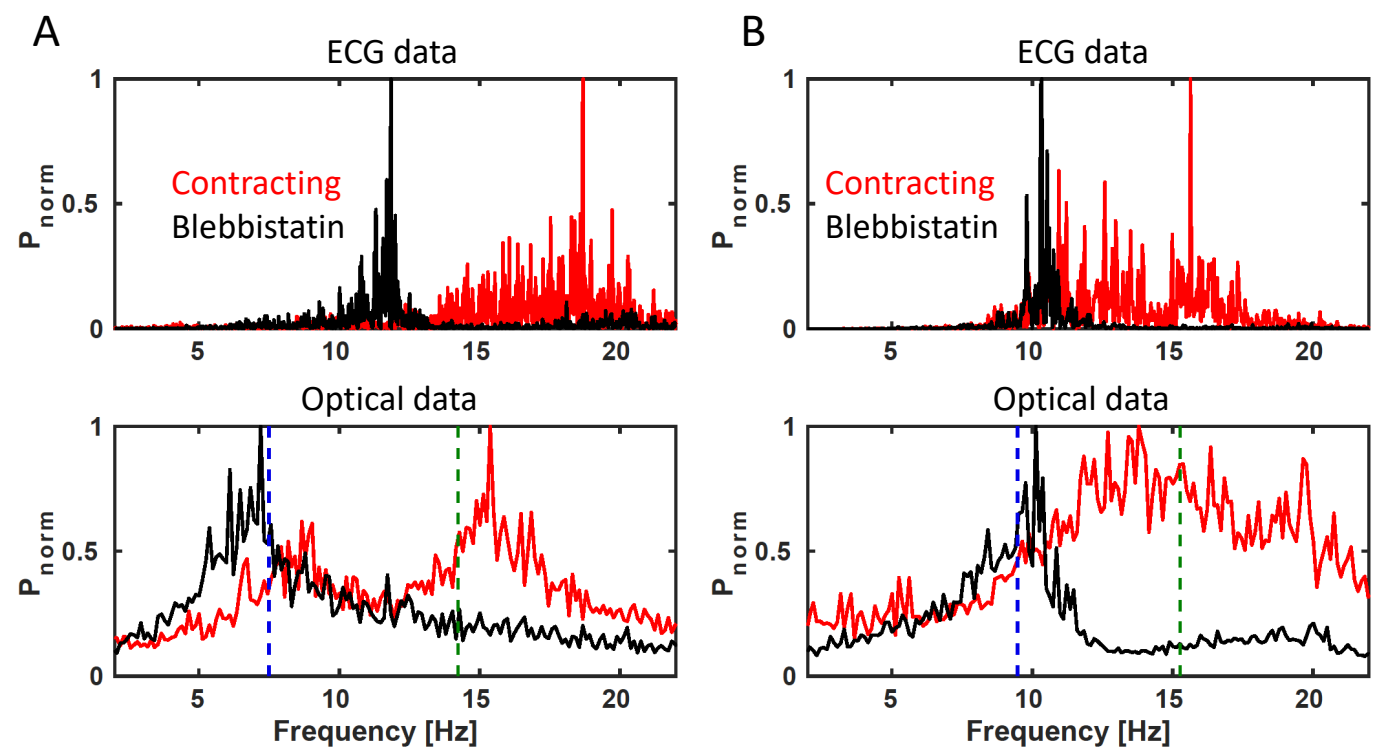

Fig. 6.12: Comparison of VF power spectra (normalized) calculated with ECG (upper row) and optical action potential (lower row) for two different rabbit hearts (panel A and B). Optical power spectra were calculated by averaging the Fourier power from all pixels used to calculate the frequency maps. Red spectra represent contracting condition, whereas black spectra correspond to Blebbistatin condition. The green and blue dashed lines represent mean values of weighted dominant frequency maps (with $P_{t h}=50 \%$ ) in contracting and Blebbistatin conditions, respectively. Overall, power spectra in contracting condition are broader than in Blebbistatin condition. The VF frequencies in contracting condition are higher in both the hearts independent of the type of signals (ECG or optical) used to calculate the frequencies.

The VF frequencies are higher in contracting hearts as compared to Blebbistatin-uncoupled hearts independent of the signal (ECG or optical) used to compute them. The peaks of the power spectra computed from optical data show significant variation from the peaks of ECG power spectra in panel $\mathrm{A}$, indicating variation among VF frequencies when computed with different types of time traces. But in panel $\mathrm{B}$, the VF frequencies do not show a noticeable change between ECG and optical action potential. Overall, VF power spectra in contracting hearts are broadened as compared to Blebbistatin-uncoupled hearts. Though the exact reason for this mismatch in frequencies computed with ECG and optical action potential in panel A 
is unknown, a possible explanation is based on the difference in recording locations of these two signals. The ECG signals are the average electrical activity recorded from the whole heart, whereas optical action potentials were recorded from the portion of heart (usually left ventricle) imaged by a single camera. Additionally, the VF frequencies need not be homogeneous on different locations of the heart as indicated by the spatial dispersion of frequencies across the heart in Figure 6.10. Since the data recorded by a single detector is used for optical mapping studies, the spatio-temporal dynamics of $\mathrm{VF}$ on other regions of the heart is unknown. Hence, for proper comparison VF frequencies using ECG and optical data, more detectors should be used to record action potential from all regions of the heart. However, both ECG and optical data show significant variation of VF frequencies between contracting and Blebbistatin-uncoupled hearts.

\begin{tabular}{|c|c|cc|cc|}
\hline \multirow{2}{*}{ Heart } & Signal & \multicolumn{2}{|c|}{ Contracting } & \multicolumn{2}{c|}{ Blebbistatin } \\
& & DF [Hz] & WDF [Hz] & DF [Hz] & WDF [Hz] \\
& & 18.69 & 18.68 & 11.84 & 11.80 \\
& ECG & 15.38 & 13.94 & 7.20 & 6.83 \\
& Optical-power average & 14.07 & 14.23 & 7.57 & 7.50 \\
& Optical-frequency map & 15.64 & 13.07 & 10.34 & 10.28 \\
& ECG & 13.79 & 15.17 & 10.14 & 9.79 \\
& Optical-power average & 15.36 & 15.27 & 9.48 & 9.48 \\
& Optical-frequency map & 15.48 & & \\
\hline
\end{tabular}

Tab. 6.1: Comparison of dominant frequencies (DF) and weighted dominant frequencies (WDF) for $N=2$ hearts computed with ECG and optical action potential time traces (using Fourier power average and frequency maps).

The frequency values computed with ECG and optical action potential time traces are tabulated in Table 6.1. Frequencies with optical action potentials were computed using averaged Fourier power spectra (Opticalpower average) and also by using frequency maps (Optical-frequency map; frequency was computed as the mean value of frequency maps). In all these three cases, the frequencies were calculated with dominant frequency (DF) and weighted dominant frequency (WDF) approaches. 


\section{Summary and discussion}

This thesis focused on the measurement of electrophysiological and mechanical properties of contracting hearts by using marker-free numerical motion tracking and ratiometric optical mapping techniques. The marker-free motion tracking substantially reduced motion artifacts due to the relative motion between the cardiac tissue and the camera, while excitation ratiometric technique (using blue and green excitation light) was used to minimize dye concentration and illumination artifacts. Illumination artifacts were minimized by arranging the blue and green light sources around the heart to achieve uniform illumination on ratio images during the experiment. The combination of these two techniques significantly removed total motion artifacts from optical action potential signals. And these techniques were used to measure electrophysiological properties such as action potential duration and electromechanical restitution, more precisely from contracting hearts in the absence of electromechanical uncouplers. Besides these, the combined motion tracking and ratiometry was applied to visualize and characterize the dynamics of ventricular fibrillation. Systematic comparisons of cardiac action potential duration, cardiac restitution and ventricular fibrillation dynamics were made in contracting and contraction-inhibited (pharmacologically with Blebbistatin) conditions.

The action potential durations $\left(\mathrm{APD}_{50}\right)$ of the contracting rabbit hearts were measured from single pixels (after smoothing) with a precision of $2.1 \pm 0.4 \mathrm{~ms}$, whereas the $\mathrm{APD}_{50}$ of Blebbistatin-uncoupled hearts were measured with a precision of $1.4 \pm 0.15 \mathrm{~ms}$. Comparison of electrophysiological parameters showed on average $27 \pm 5 \%$ ( $N=5$ hearts) shortening of action potential duration in contracting hearts compared to Blebbistatin-uncoupled non-contracting hearts. The frequency of ventricular fiibrillation was found to be significantly different between contracting $(13 \pm 3.5 \mathrm{~Hz})$ and Blebbistatinuncoupled $(8 \pm 1.5 \mathrm{~Hz})$ conditions ( $N=10$ hearts). Electrophysiological effects 
of Blebbistatin was found to be concentration-dependent and the effects were reversible by washout of Blebbistatin ( $N=2$ hearts) using fresh Tyrode's solution.

The observed differences in electrophysiological parameters between contracting and Blebbistatin-uncoupled hearts are in agreement with previous findings by Brack et al.[Bra+13] and Lee et al. [Lee+19]. Brack et al. observed a prolongation $(\approx 30 \%)$ of monophasic action potential duration $\left(\mathrm{APD}_{90}\right)$ in Blebbistatin-uncoupled condition when measured it electrically from the base and apex of New Zealand White rabbit hearts $(N=8)$ using quadripolar contact electrodes. They have also observed prolongation in optical action potential durations $(\approx 27 \%)$ from the mid-wall region of the left ventricle (less motion) in $N=4$ hearts. Another interesting observation of them is the increase in ventricular fibrillation threshold in Blebbistatinuncoupled condition. The findings shown in this thesis partially confirms their observation. Eventhough the studies shown here did not compute the threshold of VF induction, it was difficult to induce VF with Blebbistatin in $N=4$ hearts (out of $N=10$ ). On the other hand, induction of VF in contracting condition was more easy to achieve in $N=9$ hearts. Recently in 2019, Lee et al. observed prolongation $(\approx 18 \%)$ of $\mathrm{APD}_{90}$ in Langendorff perfused pig hearts in Blebbistatin-uncoupled condition using ratiometric optical mapping recordings. However, those studies were unable to remove motion artifacts originating from all the different sources as mentioned in this thesis. Additionally, those studies only measured action potentials (optical or electrical) from limited number of locations).

However, the effect of Blebbistatin on cardiac electrophysiology is still a controversial topic. During its introduction, Federov et al. [Fed+07] reported that Blebbistatin does not affect the cardiac electrophysiology. After that, multiple studies confirmed the similar findings.

Though the variations in electrophysiological parameters in contracting versus Blebbistatin-uncoupled hearts are significant, as visible in Figure 5.3 the action potentials becomes triangular at higher frequencies in contracting conditions. This indicates that heart becomes ischemic (reduction of oxygen and nutrients to the heart muscle) at this pacing frequencies. Recent study by Kuzmiak-glancy et al. $[\mathrm{Kg}+15]$ shows a correlation between shortening of action potential duration and higher metabolic demand and oxygen consumption of the hearts. Hence, the observed differences between contracting 
and Blebbistatin-uncoupled hearts might not be the direct effect of Blebbistatin, but might be an indirect effect since the metabolic demand and oxygen consumption are lower in Blebbistatin-uncoupled hearts.

\subsection{Limitations}

One of the major limitations is that optical recordings used in this thesis were performed only with a single camera. Hence, it was not possible to image the entire surface of the heart. Only $60-80 \%$ portion of the left ventricular surface $(2-3 \mathrm{~cm})$ was imaged in all the experiments. This was due to the fact that only limited number of high power LED sources were able to use owing to the limitation in the maximum voltage which the LED driver can supply (45 $V$ for each channel). Hence, only 6 high power LEDs (3 blue and 3 green) were used in the whole experiment.

Eventhough the combination of motion tracking and excitation ratiometry effectively minimizes the motion artifacts in recordings of contracting hearts,
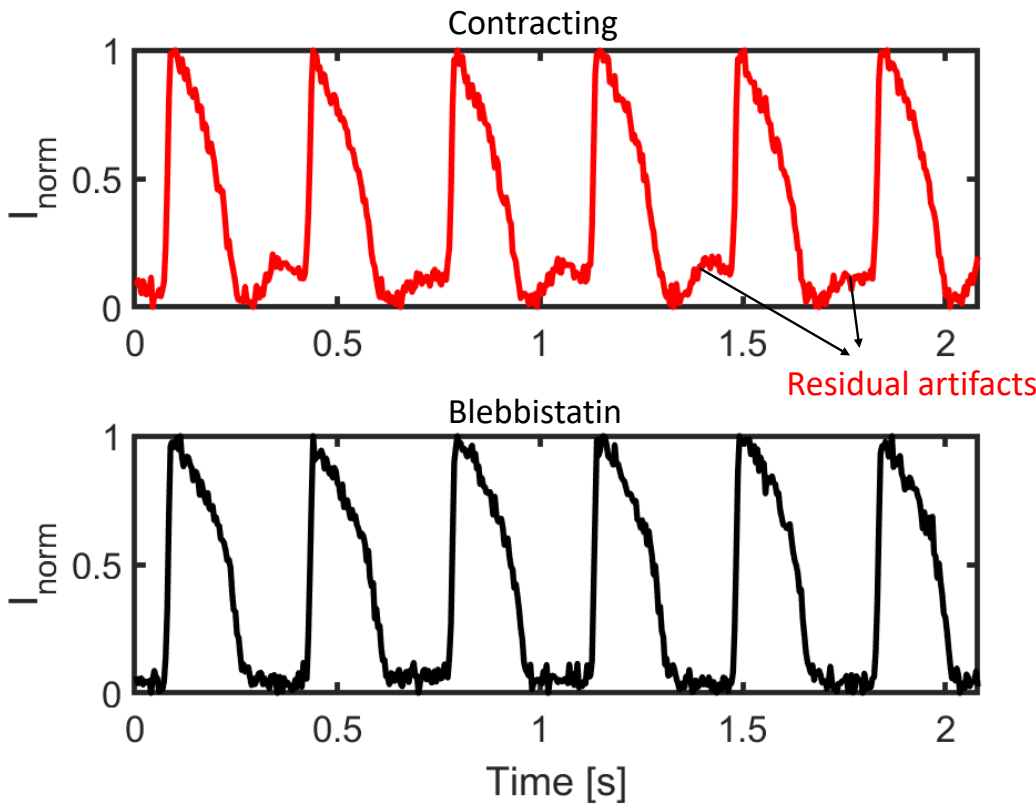

Fig. 7.1: Exemplary time traces showing residual motion artifacts in optical action potentials of a rabbit heart in contracting condition. Action potential time traces are obtained from the left ventricular surface with spatial averaging of $3 \times 3$ pixels. Time traces are normalized using a sliding window. Action potential traces in contracting condition shows severe baseline fluctuations as compared to Blebbistatin condition. 
it also has some limitations. The inability to measure action potential duration at $90 \%$ repolarization $\mathrm{APD}_{90}$ (considered as standard measurements in Blebbistatin-uncoupled hearts) from contracting hearts at all pixel locations is one of its drawbacks. This is due to the presence of residual motion artifacts (for some pixels) in the action potential signals even after applying motion tracking and ratiometry. One such example is shown in Figure 7.1. The severe base line fluctuations of the time traces due to residual artifacts cause inaccuracy in the measurement of $\mathrm{APD}_{90}$ in contracting condition.

Moreover, temporal resolution of the recorded action potential signals is half of the camera frame rate. This is due to the separation of recorded video frames (500 fps) into action potentials corresponding to blue and green excitation light (250 fps). These lowered temporal resolution can cause mild inaccuracy in the calculation of parameters such as action potential duration.

\subsection{Outlook}

Multiple LED switching boxes can be used in synchrony to produce more blue and green LED light sources which can be switched with each camera frame. This will allow excitation ratiometric measurements of optical action potential signals from the entire surface of the heart.

Replacing the excitation ratiometry with emission ratiometry can significantly reduce most of the motion artifacts originating from dye concentration and illumination inhomogeneity since in this case the optical mapping experiment uses single fluorescent dye and a single excitation wavelength. In this case, two cameras with different emission filters have to be aligned to image same location of the cardiac tissue.

While performing experiments with Langendorff perfused contracting hearts, it is necessary to improve the Tyrode's solution to compensate for the higher metabolic demand and oxygen consumption. Hence in future experiments, perfusate having higher oxygen carrying capacity such as whole blood or perfusate supplemented with red blood cells (RBCs) could be used to address the oxygenation problem of contracting hearts $[\mathrm{Kg}+15]$. 


\section{Acknowledgement}

I would like to express my deep gratitude to Prof. Ulrich Parlitz for giving me this wonderful opportunity to work on this exciting project and also for supervising and supporting in all my ups and downs for the last three and half years of my PhD life.

I would like to thank Dr. Jan Christoph for all his guidance and support throughout this project. I would like to extend my gratitude to Prof. Stefan Luther for all his valuable inputs for the completion of this work.

I would like to appreciate my thesis committee members Prof. Jörg Enderlein and Prof. Martin Uecker for their constructive feedbacks.

Many thanks goes to Marion Kunze, Claudia Richter, Tina Althaus and Laura Dias for all their technical support.

I am grateful to all my colleagues of Biomedical Physics group for their tremendous support. Special thanks to Sebastian Berg, Florian Spreckelsen, Jan Lebert, Afnan Nabizath Mohamed Nazer, Angela Gremmel, Sharmila Kubitz and Caroline Siebert.

I am expressing my sincere gratitude to Ricardo Langner for providing the cleanthesis package.

I am expressing my sincere thanks to all ITN Be-Optical members for maintaining a good scientific as well as friendly atmosphere in all our meetings. I really enjoyed it.

I would like to thank Dr. Martin James, Dr. Prashant Prabhakaran, Mohammed Ali, Subin Narayanan and Sangeeth Kochanthara for all the good time spent together.

I would like to thank Devika Rajan, my wife for all her encouragement and wishes. Finally, I am taking this time to thank my family for understanding and supporting me during the $\mathrm{PhD}$ time. 



\section{Bibliography}

[Bac+11] Andrew D. Bachtel, Richard A. Gray, Jayna M. Stohlman, et al. "A Novel Approach to Dual Excitation Ratiometric Optical Mapping of Cardiac Action Potentials With Di-4-ANEPPS Using Pulsed LED Excitation". In: IEEE transactions on biomedical engineering 58.7 (2011), pp. 2120-2126 (cit. on pp. 3, 17, 18, 20).

[BMY11] Robert M. Bell, Mihaela M. Mocanu, and Derek M. Yellon. "Retrograde heart perfusion: The Langendorff technique of isolated heart perfusion". In: Journal of Molecular and Cellular Cardiology 50.6 (2011), pp. 940950 (cit. on p. 7).

[Boo+13] Martin D Bootman, Katja Rietdorf, Tony Collins, Simon Walker, and Michael Sanderson. "Ca $2+$-Sensitive Fluorescent Dyes and Intracellular Ca 2 + Imaging”. In: 2 (2013), pp. 83-100 (cit. on p. 11).

[Bou+11] Elliot B. Bourgeois, Andrew D. Bachtel, Gregory P. Walcott, et al. "Simultaneous optical mapping of transmembrane potential and wall motion in isolated, perfused whole hearts Simultaneous optical mapping of transmembrane potential and wall motion in isolated, perfused whole hearts". In: Journal of Biomedical Optics 16.9 (2011) (cit. on pp. 2, 9, 29).

[Bra+13] Kieran E Brack, Ravi Narang, James Winter, and G André Ng. "The mechanical uncoupler blebbistatin is associated with significant electrophysiological effects in the isolated rabbit heart". In: Experimental Physiology 98.5 (2013), pp. 1009-1027 (cit. on pp. 2, 82).

[Ca192] Geert Callewaert. "Short review Excitation-contraction coupling in mammalian cardiac cells". In: Cardiovascular Research 26.10 (1992), pp. 923932 (cit. on p. 9).

[Che+00] Jay Chen, Ravi Mandapati, Omer Berenfeld, and Allan C. Skanes. "Integrative Physiology High-Frequency Periodic Sources Underlie Ventricular Fibrillation in the Isolated Rabbit Heart”. In: Circ Res. 86.1 (2000), pp. 86-93 (cit. on p. 16). 
[Chr+18] J. Christoph, M. Chebbok, C. Richter, et al. "Electromechanical vortex filaments during cardiac fibrillation". In: Nature Publishing Group 555.7698 (2018), pp. 667-672 (cit. on p. 2).

[CSSL17] J. Christoph, J. Schröder-Schetelig, and S. Luther. "Electromechanical optical mapping". In: Progress in Biophysics and Molecular Biology 130 (2017), pp. 150-169 (cit. on pp. 2, 9).

[CL18] Jan Christoph and Stefan Luther. "Marker-Free Tracking for Motion Artifact Compensation and Deformation Measurements in Optical Mapping Videos of Contracting Hearts". In: Frontiers in Physiology 9.November (2018) (cit. on pp. 2, 9, 29).

[ENS04] Igor R Efimov, Vladimir P Nikolski, and Guy Salama. "Optical imaging of the heart". In: Circulation Research 95.1 (2004), pp. 21-33 (cit. on p. 1).

[Fed+07] Vadim V. Fedorov, Ilya T. Lozinsky, Eugene A. Sosunov, et al. "Application of blebbistatin as an excitation-contraction uncoupler for electrophysiologic study of rat and rabbit hearts". In: Heart Rhythm 4.5 (2007) (cit. on pp. 2, 16, 82).

[Fra00] Michael R. Franz. "Mechano-electrical feedback". In: Cardiovascular Research 45 (2000), pp. 263-266 (cit. on p. 1).

[Fra96] Michael R. Franz. "Mechano-electrical feedback in ventricular myocardium". In: Cardiovascular Research 32.1 (1996), pp. 15-24 (cit. on pp. 1, 11).

[Gan+07] Prasanth Ganesan, Mark Sterling, Steven Ladavich, and Mark Sterling. "Computer-Aided Clinical Decision Support Systems for Atrial Fibrillation". In: IntechOpen (2007) (cit. on p. 6).

[Hor13] Daniel Hornung. "Cardiac Arrhythmia Termination on the Vascular and Organ Scale Dissertation”. PhD thesis. 2013 (cit. on p. 8).

[Kar94] Alain Karma. "Electrical alternans and spiral wave breakup in cardiac tissue". In: Chaos 4 461.June 1994 (1994) (cit. on pp. 3, 52).

[Kar93] Alain Karma. "Spiral Breakup in Model Equations of Action Potential Propagation in Cardiac Tissue". In: Physicsl Review Letters 71.7 (1993), pp. 1103-1107 (cit. on pp. 3, 52).

[Ket+04a] S. Kettlewell, N. L. Walker, S. Cobbe, F. Burton, and Godfrey L. Smith. "The electrophysiological and mechanical effects of 2,3-butane-dione monoxime and cytochalasin-D in the Langendorff perfused rabbit heart". In: Experimental Physiology 89 (2004) (cit. on p. 15). 
[Ket+04b] S Kettlewell, N L Walker, S M Cobbe, F L Burton, and G L Smith. "The electrophysiological and mechanical effects of 2 , 3-butane-dione monoxime and cytochalasin-D in the Langendorff perfused rabbit heart". In: Exp Physiol 89.2 (2004), pp. 163-172 (cit. on p. 2).

[Kni+00] Stephen B. Knisley, Robert K. Justice, W. E. I. Kong, et al. "special communication". In: Am J Physiol Heart Circ Physiol 279 (2000), pp. 14211433 (cit. on p. 18).

[Kol+98] Marcus L. Koller, Mark L. Riccio, Robert F. Gilmour, et al. "Dynamic restitution of action potential duration during electrical alternans and ventricular fibrillation". In: American Physiological Society 275 (1998), pp. 1635-1642 (cit. on p. 47).

[KP03] Peter J. Kostelec and Senthil Periaswamy. "Image registration for MRI". In: Modern Signal Processing 46 (2003), pp. 161-184 (cit. on p. 30).

[Kg+15] Sarah Kuzmiak-glancy, Rafael Jaimes Iii, Anastasia M Wengrowski, and Matthew W Kay. "Oxygen demand of perfused heart preparations : how electromechanical function and inadequate oxygenation affect physiology and optical measurements". In: 100.6 (2015), pp. 603-616 (cit. on pp. 82, 84).

[Lau+98] Kenneth R. Laurita, Steven D. Girouard, Fadi G. Akar, and David S. Rosenbaum. "Modulated Dispersion Explains Changes in Arrhythmia Vulnerability During Premature Stimulation of the Heart". In: Circulation 98 (1998), pp. 2774-2780 (cit. on p. 16).

[LS01] Kenneth R. Laurita and Ashish Singal. "Mapping action potentials and calcium transients simultaneously from the intact heart". In: Am J Physiol Heart Circ Physiol 1998 (2001), pp. 2053-2060 (cit. on p. 1).

[Lee+19] Peter Lee, Jorge G Quintanilla, Carlos Gala, et al. "In vivo ratiometric optical mapping enables high-resolution cardiac electrophysiology in pig models". In: Cardiovascular Research 115.11 (2019), pp. 1659-1671 (cit. on pp. 2, 82).

[Lee+12] Peter Lee, Fouad Taghavi, Ping Yan, et al. "In situ optical mapping of voltage and calcium in the heart". In: PLOS ONE 7.8 (2012) (cit. on p. 1).

[Liu+93] Yaning Liu, Cindido Cabo, Remy Salomonsz, Mario Delmar, and Jorge Davidenko. "Effects of diacetyl monoxime on the electrical properties of sheep and guinea pig ventricular muscle Yaning". In: Cardiovascular Research 27 (1993), pp. 1991-1997 (cit. on p. 2). 
[Liu +92] Yaning Liu, Cándido Cabo, Remy Salomonsz, et al. "Effects of diacetyl monoxime on the electrical properties of sheep and guinea pig ventricular muscle". In: Cardiovascular Research 27 (1992), 1991-1997 (cit. on p. 15).

[LLE12] Qing Lou, Wenwen Li, and Igor Efimov. "The role of dynamic instability and wavelength in arrhythmia maintenance as revealed by panoramic imaging with blebbistatin vs. 2,3-butanedione monoxime". In: American journal of physiology. Heart and circulatory physiology 302 (Jan. 2012), H262-9 (cit. on p. 15).

[Man+98] Ravi Mandapati, Yukio Asano, William T Baxter, Richard Gray, and Jorge Davidenko. "Quantification of Effects of Global Ischemia on Dynamics of Ventricular Fibrillation in Isolated Rabbit Heart". In: Circulation. (1998), pp. 1688-1697 (cit. on p. 16).

[Min11] Mina Attin, William T Clusin. "Basic Concepts of Optical Mapping Techniques in Cardiac Electrophysiology". In: Biol Res Nurs. 11.2 (2011), pp. 195-207 (cit. on p. 1).

[Mon14] Mahdis Monajati. "The Validity Value of Electrical Restitution Curve in Prediction of Ventricular Fibrillation Induction Using ANFIS". In: 3.2 (2014), pp. 178-184 (cit. on p. 3).

[Ng16] G André Ng. "Feasibility of selection of antiarrhythmic drug treatment on the basis of arrhythmogenic mechanism - Relevance of electrical restitution, wavebreak and rotors". In: Pharmacology and Therapeutics (2016) (cit. on p. 3).

[Nid+98] Pedro J D E L Nido, Paul Glynn, Percival Buenaventura, et al. "special communication". In: Am J Physiol Heart Circ Physiol (1998), pp. 728-741 (cit. on p. 1).

[Osa12] Oleg E Osadchii. "Effects of ventricular pacing protocol on electrical restitution assessments in guinea-pig heart". In: Experimental Physiology 97.7 (2012), pp. 807-821 (cit. on p. 47).

[PF03] Senthil Periaswamy and Hany Farid. "Elastic registration in the presence of intensity variations". In: IEEE Transactions on Medical Imaging 22.7 (2003), pp. 865-874 (cit. on p. 30).

[PT14] Emily R Pfeiffer and Jared R Tangney. "Biomechanics of Cardiac Electromechanical Coupling and Mechanoelectric Feedback". In: Journal of Biomechanical Engineering 136.February (2014), pp. 1-11 (cit. on pp. 9, 11). 
[Raw16] Rawan A. Khuwaileh. "Electrical restitution and action potential repolarisation studies in acutely isolated cardiac ventricular myocytes Thesis submitted for the degree of Doctor of Philosophy at the University of Leicester By Rawan A . Khuwaileh , MD Department of Molecular". PhD thesis. 2016 (cit. on p. 47).

[RKG99] Mark L Riccio, Marcus L Koller, and Robert F Gilmour. "Electrical Restitution and Spatiotemporal Organization During Ventricular Fibrillation". In: Circulation Research 84 (1999), pp. 955-963 (cit. on p. 3).

[SC00] Guy Salama and Bum-Rak Choi. "Images of Action Potential Propagation in Heart”. In: News Physiol. Sci. 15.February (2000), pp. 33-41 (cit. on p. 1).

[Sir96] Max J Lab Sirfraz A. Nazir. "Mechanoelectric feedback and atria1 arrhythmias". In: Cardiovascular Research 32.1 (1996), pp. 52-61 (cit. on pp. 1, 11).

[Tse +16] Gary Tse, B A Hons, Mbbs Ma, et al. "Cardiac dynamics : Alternans and arrhythmogenesis". In: Journal of Arrhythmia 32 (2016), pp. 411-417 (cit. on p. 52).

[Val+02] Miguel Valderrábano, Junzhong Yang, Chikaya Omichi, et al. "Frequency Analysis of Ventricular Fibrillation in Swine Ventricles". In: Circulation Research 90 (2002), pp. 213-222 (cit. on p. 65).

[Ven+12] Raghav Venkataraman, Mark R Holcomb, Rene Harder, Björn C Knollmann, and Franz Baudenbacher. "Ratiometric imaging of calcium during ischemia-reperfusion injury in isolated mouse hearts using Fura-2". In: BioMedical Engineering OnLine Mi (2012), pp. 1-16 (cit. on p. 1).

[Wei+02] James N Weiss, Peng-sheng Chen, Zhilin Qu, and Alan Garfinkel. "Electrical Restitution and Cardiac Fibrillation". In: Journal of cardiovascular electrophysiology 13 (2002) (cit. on p. 3).

[Xie+04] Fagen Xie, Zhilin Qu, Junzhong Yang, et al. "A simulation study of the effects of cardiac anatomy in ventricular fibrillation". In: Journal of Clinical Investigation 113.5 (2004), pp. 686-693 (cit. on p. 3).

[Zha +16] Hanyu Zhang, Kenichi Iijima, Jian Huang, Gregory P. Walcott, and Jack M. Rogers. "Optical Mapping of Membrane Potential and Epicardial Deformation in Beating Hearts". In: Biophysical Journal 111.2 (2016), pp. 438-451 (cit. on pp. 2, 9, 19, 29). 